\title{
Parallel Domain Decomposition Based Power System Simulation
}

\author{
by \\ Anda Zhao, B.Eng. \\ A thesis submitted to the \\ Faculty of Graduate and Postdoctoral Affairs \\ in partial fulfillment of the requirements for the degree of
}

Master of Applied Science in Electrical Engineering

Ottawa-Carleton Institute for Electrical and Computer Engineering

Department of Electronics

Carleton University

Ottawa, Ontario

May, 2018

(C) Copyright

Anda Zhao, 2018 
The undersigned hereby recommends to the

Faculty of Graduate and Postdoctoral Affairs acceptance of the thesis

\title{
Parallel Domain Decomposition Based Power System Simulation
}

\author{
submitted by Anda Zhao, B.Eng. \\ in partial fulfillment of the requirements for the degree of
}

Master of Applied Science in Electrical Engineering

Professor Niall Tait, Chair,

Department of Electronics

Ottawa-Carleton Institute for Electrical and Computer Engineering

Department of Electronics

Carleton University

May, 2018 


\section{Abstract}

With the continuous developing of power systems, simulation of modern systems requires more computational resources and more simulation time. Conventional techniques are not efficient due to the large size of equation set and inconvenient modelling methods. Although computing power has increased dramatically, we still need new algorithms to improve the simulation speed.

To address the above problems, this thesis proposes an efficient parallel calculation algorithm based on domain decomposition method. The new approach reduces the computational cost by calculating in parallel and splitting the equation set. The proposed approach partitions the system into several sub-systems to be solved in parallel on different processors and at the same time preserves the details of the original system. To reduce the cost of communication overhead and computation load, latency characteristic is utilized. Not all the Jacobian matrix is updated in every iteration. Traditional simulators (transient stability and electromagnetic transient) as well as the hybrid simulator are described and compared with this algorithm. Computational examples are used to validate the accuracy of new proposed algorithm and its efficiency. 


\section{Acknowledgments}

I would like to express my gratitude to my supervisor, Professor Xiaoyu Wang for his encouragement, suggestion and guidance in my hard time. Without his help, it is hard for me to finish this thesis. I appreciate the time of Professor Xiaoyu Wang spending on my modelling and design. At the meantime, I learned a lot from him about how to become an engineer and think as a researcher.

Secondly, I would like to give my gratitude to my co-supervisors, Professor Michel Nakhla and Dr. Behzad Nouri, to their consistent and illuminating instruction. This thesis could not be finished without their knowledge and help. They also helped a lot on my writing.

I would also like to thank my colleagues in my group who help me a lot on technique problems. Without their valuable help and suggestions, the thesis could not have been possible. They are truly great friends in my life.

Last but not least, I would like to give my special thanks to my family, especially

my parents for their unconditional love and support. Thanks to their support, I can pay all the attention to my research. 


\section{Table of Contents}

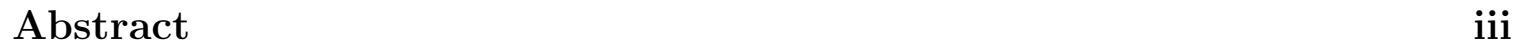

Acknowledgments iv

\begin{tabular}{ll}
\hline Table of Contents & v
\end{tabular}

List of Tables ix

List of Figures $\quad$ X

List of Acronyms xiii

List of Symbols $\quad$ XV

\begin{tabular}{lll}
\hline 1 & Introduction & 1
\end{tabular}

1.1 Background and Motivation . . . . . . . . . . . . . . . . . 1

1.2 Objectives and Contributions . . . . . . . . . . . . . . . . 3

1.3 Organization of the Thesis . . . . . . . . . . . . . . . . . . . 3

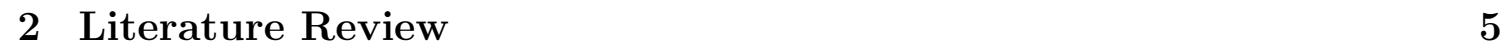

2.1 Objectives of Power System Simulation . . . . . . . . . . . . . 5

2.1 .1 Power System Stability . . . . . . . . . . . . . . . . . 6

2.2 Fundamentals of Conventional Simulators . . . . . . . . . . . . . . . . . 7 
2.2 .1 Traditional Simulation Studies . . . . . . . . . . . . . . 7

2.2 .2 Hybrid Simulation of Power System . . . . . . . . . . . . 11

2.3 Numerical Example . . . . . . . . . . . . . . . . . . . . . . . . . . . . 17

2.4 Recent Developments $\ldots \ldots \ldots \ldots \ldots$

\begin{tabular}{|ll|}
\hline 3 & Power System Components Modelling \\
\hline
\end{tabular}

3.1 Formulation of System Equations . . . . . . . . . . . . . . . . 23

3.2 Park and Clark Transformation . . . . . . . . . . . . . . . . . 24

3.3 Modelling of Synchronous Generator . . . . . . . . . . . . . 26

$3.3 .1 \quad$ IEEE Type 1 Execitor Model $\ldots \ldots \ldots \ldots$. . . . . . 27

3.3 .2 Governor Model . . . . . . . . . . . . . . . . . . . . . . 28

$3.3 .3 \quad \mathrm{DQ}-\mathrm{abc}$ interface $\ldots \ldots \ldots \ldots \ldots$

3.4 Modelling of Wind Energy Conversion System . . . . . . . . . . . 30

3.4 .1 Aerodynamic and Mechanical Systems $\ldots \ldots \ldots$

$3.4 .2 \quad \mathrm{PMSG} \ldots \ldots \ldots \ldots \ldots$

3.4 .3 Generator Side Controller . . . . . . . . . . . . . . . . 33

3.4 .4 Grid Side Controller . . . . . . . . . . . . . . . . . 34

3.4 .5 Modelling of AC-DC-AC Voltage Source Converter(VSC) . . . 36

3.5 Transmission Line. . . . . . . . . . . . . . . . . . . . . . . . . . . 37

3.6 Loads . . . . . . . . . . . . . . . . . . . . . . . . . . . . . . 39

\begin{tabular}{|lll}
4 & Parallel Domain Decomposition Method & 40
\end{tabular}

$4.1 \quad$ Domain Decomposition $\ldots \ldots \ldots \ldots$

4.2 Non-overlapping Domain Decomposition Method $\ldots . . . . . . . .44$

$4.2 .1 \quad$ Schur Complement Method $\ldots \ldots \ldots$

4.3 Parallel Implementation $\ldots \ldots \ldots \ldots$ 
4.4 Special Characteristics of Domain Decomposition Applied to Power System Simulation . . . . . . . . . . . . . . . . 56

4.4 .1 System Partitioning . . . . . . . . . . . . . . . 56

4.4 .2 Latency . . . . . . . . . . . . . . . . . . . . . 58

4.4 .3 Initialization . . . . . . . . . . . . . . . 60

\begin{tabular}{|lll|}
\hline 5 & Simulation Result Analysis and Comparison & 64
\end{tabular}

5.1 Numerical Stability . . . . . . . . . . . . . . . . . . . 65

5.2 Performance Index . . . . . . . . . . . . . . . . . . . . . . . . . . 66

5.2 .1 Accuracy Index . . . . . . . . . . . . . . . . 66

5.2 .2 Efficiency Index . . . . . . . . . . . . . . . . . 66

5.3 Case Study I: Small-Scale Power System . . . . . . . . . . . . . . . . 67

5.3 .1 Accuracy $\ldots \ldots \ldots \ldots 7$

5.3 .2 Speed Performance . . . . . . . . . . . . . . . . . . . 70

5.4 Case Study II: Medium-Scale Power System . . . . . . . . . . . . . . 71

$5.4 .1 \quad$ Accuracy $\ldots \ldots \ldots \ldots . \ldots \ldots \ldots 71$

5.4 .2 Speed Performance . . . . . . . . . . . . . . . 75

5.5 Case Study III: Large-Scale Power System . . . . . . . . . . . . . . . 75

5.5 .1 Accuracy . . . . . . . . . . . . . . . . 76

5.5 .2 Speed Performance . . . . . . . . . . . . . . . . . . 79

5.6 Case Study IV: Large-Scale Power System . . . . . . . . . . . . . . . 80

5.6 .1 Accuracy . . . . . . . . . . . . . . . . . . 80

5.6 .2 Speed Performance . . . . . . . . . . . . . . . 82

5.7 Speed-Up Analysis $\ldots \ldots \ldots$. . . . . . . . . . . . . . 83

5.8 Analysis of Multi-cores Simulation . . . . . . . . . . . . . . . . . 85 
\begin{tabular}{|lll}
\hline 6 & Conclusion and Future Work & 87
\end{tabular}

6.1 Conclusions . . . . . . . . . . . . . . . . . . . . 87

6.2 Future Work . . . . . . . . . . . . . . . . . . . . . . . . . . . . . . . . . 88

\begin{tabular}{lr}
\hline List of References & 90
\end{tabular}

\begin{tabular}{|ll}
\hline Appendix A Derivation of Power Flow & 97
\end{tabular}

A.1 Power Flow Calculation . . . . . . . . . . . . . . . . . . . . . 97

A.1.1 Bus classification . . . . . . . . . . . . . . . . . . . . 97

A.1.2 Newton-Raphson solution method . . . . . . . . . . . . . . . 98

\begin{tabular}{|ll}
\hline Appendix B Parameters of the Study Cases & 100
\end{tabular}

B.1 IEEE 9 bus system . . . . . . . . . . . . . . . . . . . . . . . . . . . 100

B.2 IEEE 39 bus system . . . . . . . . . . . . . . . . . . . . . . . . 102

B.3 IEEE 118 bus system . . . . . . . . . . . . . . . . . . . . . . . . . . . 105

B.4 IEEE 2869 bus system . . . . . . . . . . . . . . . . . . . . 106 


\section{List of Tables}

$2.1 \quad$ EMT model for inductors and capacitors $\ldots \ldots \ldots \ldots \ldots$

$5.1 \quad$ CPU time of IEEE 9 bus system $\ldots \ldots \ldots \ldots \ldots \ldots \ldots$

5.2 CPU Time of IEEE 39 bus system $\ldots \ldots \ldots \ldots \ldots \ldots$

$5.3 \quad$ CPU Time of IEEE 118 bus system $\ldots \ldots \ldots \ldots \ldots$

5.4 Examples of IEEE 2869 bus system power flow calculation . . . . . 81

$5.5 \quad$ CPU Time of IEEE 2869 bus system $\ldots \ldots \ldots \ldots \ldots \ldots$

B.1 Transmission line characteristics of IEEE 9 bus system . . . . . . . 101

B.2 Parameter for each synchronous machines in IEEE 9 bus system . . . 101

\begin{tabular}{|lll}
\hline B.3 & Examples of Transmission line characteristics of IEEE 39 bus system & 103
\end{tabular}

B.4 Transmission line characteristics of IEEE 118 bus system . . . . . . . 106

B.5 Transmission line characteristics of IEEE 2869 bus system … . . 106 


\section{List of Figures}

2.1 Classification of power system stability $\ldots \ldots \ldots$. . . . . . . . . . 7

2.2 EMT equivalent circuit . . . . . . . . . . . . . . . . . . 10

2.3 Block diagram of control system in EMT . . . . . . . . . . . . . . . . 11

2.4 Overview of hybrid simulator . . . . . . . . . . . . . . 13

2.5 Serial interaction protocol of hybrid simulator . . . . . . . . . . 15

2.6 Parallel interaction protocol of hybrid simulator . . . . . . . . . . 16

$2.7 \quad$ Detailed system along with the external system equivalent $\ldots$. . . . . 17

2.8 Comparison between hybrid simulator and EMT simulator (Voltage). 18

2.9 Comparison between hybrid simulator and EMT simulator (Current) 19

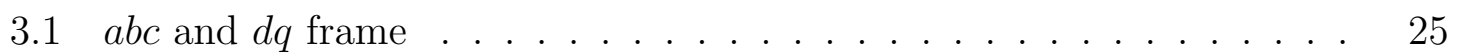

3.2 IEEE Type 1 exciter block diagram . . . . . . . . . . . . . . . . 28

3.3 Governor block diagram . . . . . . . . . . . . . . . . . . . . . . . . . 28

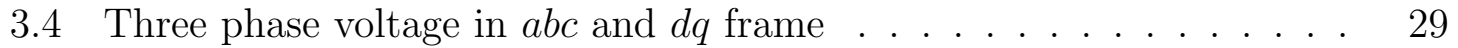

3.5 Block diagram of WECS . . . . . . . . . . . . . . . . . . . . . . . . . 30

3.6 Turbine characteristic curves . . . . . . . . . . . . . . . . . . . . . . 32

3.7 Control block for the generator side converter . . . . . . . . . . . . . 33

3.8 Control block for the grid side converter . . . . . . . . . . . . . 35

3.9 Circuit diagram of two-level voltage source converter . . . . . . . . . 37

3.10 Average model of VSC . . . . . . . . . . . . . . . . . . 37 
3.11 Comparison of average model and detailed model(DC link) . . . . . 38

3.12 Transmission line $\pi$ model $\ldots \ldots \ldots \ldots$

$4.1 \quad$ Block diagram of original whole system $\ldots \ldots \ldots \ldots$

$4.2 \quad$ Non over lapping partition $\ldots \ldots \ldots \ldots \ldots$

$4.3 \quad$ Overlapping partition $\ldots \ldots \ldots \ldots \ldots \ldots$

4.4 Example of system decomposition $\ldots \ldots \ldots \ldots \ldots$

$4.5 \quad$ Sparsity pattern of original system $\ldots \ldots \ldots \ldots$

$4.6 \quad$ Sparsity pattern of partitioned system $\ldots \ldots \ldots \ldots$

4.7 The Parallel Domain Decomposition Algorithm . . . . . . . . . . 54

4.8 Spmd operation mechanism with four processors $\ldots \ldots \ldots \ldots \ldots$

$4.9 \quad$ Example of partition of IEEE 39 bus system $\ldots \ldots \ldots \ldots \ldots$

4.10 Decomposed power system $\ldots \ldots \ldots \ldots \ldots$

4.11 Example of new partitioning method $\ldots \ldots \ldots \ldots$

4.12 Phasor diagram of synchronous machine in steady state . . . . . . . 62

4.13 The Parallel Domain Decomposition Algorithm with latency . . . . . 63

$5.1 \quad$ Voltage transient change at bus $1 \ldots \ldots \ldots \ldots$

$5.2 \quad$ Voltage transient change at bus 1 (zoom in) $\ldots \ldots \ldots \ldots$

$5.3 \quad$ Voltage transient change at bus $6 \ldots \ldots \ldots \ldots$

5.4 Voltage transient change at bus 4 by TS simulator . . . . . . . 72

$5.5 \quad$ Voltage transient change at bus $4 \ldots \ldots \ldots \ldots \ldots \ldots$

$5.6 \quad$ Voltage transient change of bus 4 during fault (zoom in) . . . . . . 74

5.7 Voltage transient change of bus 4 after fault clear (zoom in) . . . . 74

5.8 Steady state voltage on bus $17 \ldots \ldots \ldots \ldots \ldots$

5.9 Transient voltage on bus $17 \ldots \ldots \ldots \ldots \ldots \ldots$

5.10 Voltage transient change of bus 17 during fault $\ldots \ldots \ldots \ldots 78$

5.11 Voltage transient change of bus 17 after fault clear. . . . . . . 78 
5.12 Voltage transient change of bus 6163 . . . . . . . . . . . . . . . . . . 81

5.13 Speedup of algorithm DD . . . . . . . . . . . . . 86

B.1 IEEE standard nine bus system . . . . . . . . . . . . . . . . . . . . . 100

B.2 IEEE standard thirty-nine bus system . . . . . . . . . . . . . . . . . 102

B.3 IEEE standard one hundred eighteen bus system . . . . . . . . . . . . 105 


\section{List of Acronyms}

\begin{tabular}{ll} 
Acronyms & Definition \\
\hline \hline VSC & Voltage Source Converter \\
HVDC & High Voltage Direct Current \\
LCC & Line Commutated Converter \\
DD & Domain Decomposition \\
TS & Transient Stability \\
EMT & Electromagnetic Transient \\
DAE & Differential-Algebraic Equations \\
CPU & Central Processing Unit \\
RAM & Random Access Memory \\
NR & Newton - Raphson \\
& \\
& \\
MNE & \\
&
\end{tabular}




\begin{tabular}{ll} 
PGNME & Parallel General Norton with Multi-port Equivalent \\
BBDF & hierarchical block bordered diagonal form \\
DPIM & Dynamic Phasor based Interface Model \\
PLL & Phase Lock Loop \\
WECS & Wind Energy Conversion System \\
MPPT & Maximum Power Point Tracking \\
PMSG & Permanent Magnetic Synchronous Generator \\
DPC & Direct Power Control \\
PWM & Pulse Width Modulation \\
PDE & Partial Differential Equations \\
Parfor & parallel for-loop \\
SPMD & single program multiple data \\
PDD & Parallel Domain Decomposition \\
PDDL & Parallel Domain Decomposition with Latency \\
NDD & New Partition based Parallel Domain Decomposition \\
\hline
\end{tabular}




\section{List of Symbols}

\section{Symbols Definition}

\begin{tabular}{ll}
\hline \hline $\boldsymbol{G}$ & matrix describing the memoryless elements in the system \\
$h$ & matrix describing the memory elements in the system \\
$\mathrm{H}$ & time step of the transient simulation \\
$D$ & damping factor \\
$x_{d}$ & reactance of the d-axis \\
$x_{q}$ & reactance of the q-axis \\
$x_{d}^{\prime}$ & subtransient reactance of the d-axis \\
$T_{q 0}^{\prime}$ & Subtransient reactance of the q-axis \\
$T_{q}^{\prime}$ & d-axis open-circuit time constant \\
&
\end{tabular}




$\begin{array}{ll}\beta & \text { pitch angle of PMSG } \\ \rho & \text { tip speed ratio } \\ \psi & \text { density of air } \\ \psi & \text { the flux constant } \\ N_{p} & \text { pole pairs of PMSG } \\ \Gamma & \text { interface between each sub-domains } \\ N_{i} & \text { the number of unknowns of subsystem } i \\ N_{s} & \text { the number of unknows in interface } \\ \boldsymbol{J} & \text { Jacobian matrix } \\ \tilde{\boldsymbol{R}} & \text { Schur complement matrix } \\ \boldsymbol{C}, \boldsymbol{D} & \text { interface coupling matrix } \\ & \end{array}$




\section{Chapter 1}

\section{Introduction}

This chapter introduces the background and motivation of this thesis and presents the main contributions.

\subsection{Background and Motivation}

Presently, with the increasing demands of green and renewable energy, more and more wind power plants and solar panels are connected into the grid. Traditionally, wind energy connects to the grid through voltage source converter based high-voltage

dc (VSC-HVDC) [1]. Therefore, transmission systems have to integrate increasing numbers of power sources [2]. For example, there are more than fifty LCC-HVDC transmission lines being constructed to deliver more wind power in China [3]. In this thesis, based on the domain decomposition (DD) method, we propose a new fast and accurate simulation method to study the stability of power systems and to analyze the corresponding fault response.

Power system dynamic simulations are basically classified into two categories: Transient Stability (TS) study and Electromagnetic Transients (EMT) study. Transient stability focuses on planning large power system with several thousands of buses 
and machines [4]. As a result, the time-step in the range of milliseconds (ms) is chosen to meet simulation speed requirements. There is an assumption that only behaviour of one phase at fundamental frequency is considered. All the equations are modelled and solved in frequency domain. The remaining two phases should be $120^{\circ}$ lagging/leading of simulation result. Frequency domain solution cannot describe transient change very well. EMT simulation is mainly used to design power system which involves electronic components and protection components [5]. Phasor model cannot describe electronics components any more. EMT equations are modelled in time domain, therefore the applicable frequency range would be large. These electric components(machines, converters and wind turbines) are described by non-linear Differential and Algebraic Equations(DAEs). Meanwhile, the network is represented by linear differential and algebraic equations with history value. The time-step is in the range of microseconds(us), which is much smaller than switching time to meet accuracy requirements. These equations try to describe all details and dynamic changes. Hundreds of thousands of equations may be used to describe a large-scale power system. A large equation set and small time step must lead to huge computation load.

The above two methods are widely used in some commercial programs, such as Simulink, PSCAD/EMTP and Hypersim. In these programs, the speed of simulation is always a critical factor to reduce cost and increase productivity. During the last decade, the development of Central Process Unit(CPU) and Random Access Memory (RAM) has led to an acceleration of simulation speed. However, the size of power grid or the complexity of simulation has also grown a lot at the same time. The demands for a large number of detailed synchronous machines and converters can easily exhaust the resource of computer [9]. To find the balance between TS and EMT, hybrid simulation is implemented. Hybrid simulator inherits the advantages and disadvantages 
of above two simulators, which means its simulation efficiency is fast but accuracy is sacrificed. In addition, hybrid simulation needs complex preparation, including TS modelling, EMT modelling, equivalent modelling and interface development. The appearance of parallel computation can solve these problems effectively and boost simulation performance. The implementation of parallel computation are based on the field of DD methods. With the help of this proposed method, a new simulator with higher efficiency and higher accuracy is developed.

\subsection{Objectives and Contributions}

The objective of this thesis is to apply and assess a novel methodology for the power system simulation. The main contributions in this thesis are as follows:

- Implementing MNA method on the grid can handle linear and nonlinear circuits in both frequency and time domains;

- Domain Decomposition method with parallel calculation is applied to the power system to increase simulation speed;

- Implementing latency to the subsystem whose time constant is large to increase simulation speed.

These contributions lead to a new parallel simulation method for power system which ensures the accurate result and simulation speed.

\subsection{Organization of the Thesis}

The rest of this thesis is organized as follows: in Chapter 2 , the significance of power system simulation is discussed. Meanwhile, general methods and basic definitions 
which are related to simulation will also be reviewed. The different types of conventional simulators are discussed. In Chapter 3 , the modelling process of power system and each components (e.g., converters and machines) will be described. In Chapter 4. a new proposed algorithm is presented, which is parallel domain decomposition method. The implantation process and characteristics are presented in detail. In the following Chapter 5, four different power systems are used to demonstrate and validate the proposed method. Finally, Chapter 6 gives a conclusion and future research opinions. 


\section{Chapter 2}

\section{Literature Review}

\subsection{Objectives of Power System Simulation}

In this chapter, general terms and definitions which are related to power system simulation will be reviewed. Power system simulation mainly means the simulation of power transmission system. The interconnection lines between a generating site and an electrical substation are known as a transmission network. The local wiring between high voltage substations and customers is typically referred to as electric power distribution system. And this power distribution system is already summarized and included in the transmission system. The simulation of transmission system is more important and harder than the simulation of distribution system. Therefore this thesis focuses on the simulation of power transmission system. Dynamic simulations are frequently used in education and industry to check the stability of electric power system after facing disturbances. Stability is crucial for the operation of electric power system. We cannot plan, design and operate an electric power system without testing it. Power system companies analyze large scale power systems' stability based on simulation results. Therefore, faster and more accurate simulation methods are what we are seeking for. Literature review is necessary to analyze the characteristic 
of existing technology.

Several techniques are currently available to conduct simulations of power systems. A transient stability based simulator runs a power system in phasor domain and can provide high speed simulation results. An electromagnetic transient based simulator models a power system in detail and can provide accurate simulation results. This chapter explores these two conventional methods and introduces a hybrid simulator.

The rest of this chapter is organized as follows: Section. 2.2 gives the definitions of power system stability and explores the traditional two simulation methods in power system. Section. 2.2.2 describes the new hybrid simulator in power system. In the end, hybrid simulation is compared with traditional methods.

\subsubsection{Power System Stability}

Power system stability has been recognized as a very important role since the 1920s. Once the power system loses stability, the damage must be large and irreversible such as Northeast power failure in 1965 in United States [10]. In history, transient stability is the dominant stability problem on the most power systems. Recently, voltage stability, frequency stability and rotor angle stability attract more concerns than the past. A clear understanding of each type is essential for the design and developing of power systems. There are several papers discussing about the definition of stability [11, 12], but none of them can completely describe the whole problem. In [13], the authors give the definition of power system stability.

- Power system stability is the ability of an electric power system to regain a state of operating equilibrium after receiving a disturbance (e.g. lightning).

The stability is classified as shown in Figure. 22.1, which is based on the system variables in which instability can be observed. For example, rotor angle stability 
means the ability of synchronous machine to keep synchronism after being attacked by a disturbance. It mainly depends on the ability to maintain the balance between mechanical torque and electrical torque. Similarly, voltage stability and frequency stability mean the ability to keep voltage and frequency stable. Voltage stability mainly depends on the balance between load supply and load demand. Frequency stability mainly depends on the balance between power generation and power consumption.

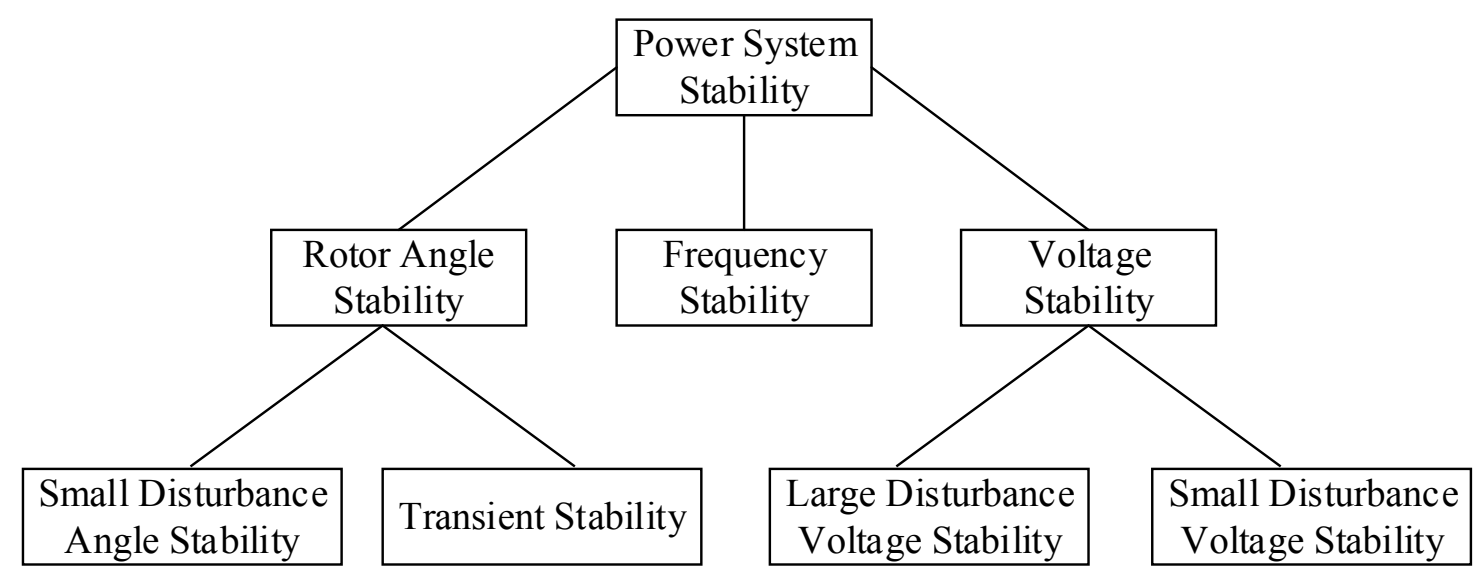

Figure 2.1: Classification of power system stability

\subsection{Fundamentals of Conventional Simulators}

\subsubsection{Traditional Simulation Studies}

Many papers discussed about traditional simulation methods for steady state and transient change in the past decades. TS and EMT simulations are the two most important analysis methods in power systems. These two methods study the design for different study goals. 


\section{Transient Stability Study}

The TS simulator, as being a fast and fuzzy simulator, uses a set of one-order differential equations and a set of complex algebraic equations to describe the whole power system.

$$
\begin{gathered}
\boldsymbol{X}^{\prime}=f(X, V) \\
\boldsymbol{I}(X, V)=\boldsymbol{Y}(X) \boldsymbol{V}
\end{gathered}
$$

where $\boldsymbol{X}$ represents the generators state variables, vector $\boldsymbol{V}$ is the bus voltage, vector $\boldsymbol{I}$ is the injected current, and $\boldsymbol{Y}$ represents the grid admittance matrix. The differential equations represent the synchronous machines while complex algebraic equations model the grid including transmission lines and loads. An implicit integration method like trapezoidal method can be used to convert differential equations to algebraic equations. After that, all the equations are algebraic and can be lumped together. Newton-Raphson(NR) iterative method is used to solve the nonlinear algebraic equations. Discrete (2.1) with time step $h$ by using Trapezoidal rule [14]:

$$
\boldsymbol{X}[n]=\boldsymbol{X}[n-1]+\frac{h}{2}(f(X[n], V[n])+f(X[n-1]+V[n-1]))
$$

where $\mathrm{n}=1,2,3 \ldots$ represent the time instants.

Rewrite the (2.2) and 2.3 in the form of function:

$$
\begin{aligned}
& f_{1}=\boldsymbol{X}[n]-\boldsymbol{X}[n-1]-\frac{h}{2}(f(\boldsymbol{X}[n], \boldsymbol{V}[n])+f(\boldsymbol{X}[n-1]+\boldsymbol{V}[n-1])) \\
& f_{2}=I(\boldsymbol{X}[n], \boldsymbol{V}[n])-Y(\boldsymbol{X}[n]) * \boldsymbol{V}[n]
\end{aligned}
$$

Using the NR method in appendix for 2.4. The final solution can be obtained. 


\section{Electromagnetic Transient Study}

Another method which can give more details on the voltage and current transient change is called electromagnetic transient. In general, EMT uses conductance and current source to replace inductors, capacitors and other memory components [8]. The relationship between voltages and currents of most of the power system components is governed by a set of ordinary differential equations. Numerical solution of these differential equations is essentially a step by step integration of these equations with a solution step that is equal to the time-step of the simulation. Numerical integration is also used to transform the differential equations of each system component into algebraic equations, with time-dependent voltages/currents relationships, that involves past (history) values of voltages and/or currents [8]. For the sake of illustration, consider the simple first-order ordinary differential equation of inductor.

$$
u(t)=L \frac{d i(t)}{d t}
$$

Based on trapezoidal method, Eq. 2.5 is given by:

$$
i(t)=G u(t)+i_{t}(t-h)
$$

where $G$ is equivalent conductance value, $i_{t}$ is the history value which is the function of $u$ and $i$. An equivalent circuit corresponding to (2.6) can be built as Figure. 2.2. Here, $G=\frac{h}{2 L}$ and $i_{t}=G u(t-h)-i(t-h)$. Figure. 2.2 shows that the equivalent circuit is consisting of conductance and current source whose value is $i_{t}$. The history current depends on the previous time-step value and conductance $G$. After converting all the components into EMT model, the system now is made up of resistance and current source. Nodal analysis can be applied easily. The nodal equations for the 


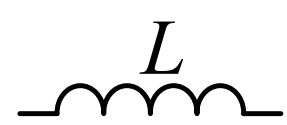

(a) Inductor

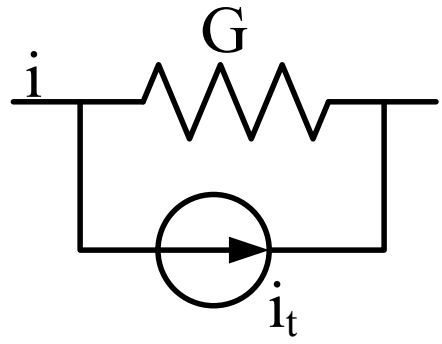

(b) Equivalent Circuit

Figure 2.2: EMT equivalent circuit

network is shown in 2.7

$$
\boldsymbol{G} \boldsymbol{v}(t)=\boldsymbol{i}(t)-\boldsymbol{I}_{h i s t}
$$

where $\boldsymbol{G} \in \mathbb{R}^{n \times n}$ is the admittance matrix, $\boldsymbol{v}(t) \in \mathbb{R}^{n}$ is the node voltage, $\boldsymbol{i}$ is the injected current and $\boldsymbol{I}_{\text {hist }}$ is the history current. The admittance matrix G remains constant during each time-step and right hand side part of (2.7) updates every time-step. Even when switches exists in the network, the admittance matrix remains constant. The summary of relationship between admittance, timestep and component value is shown in Table. 2.1.

Table 2.1: EMT model for inductors and capacitors

\begin{tabular}{|c||c|c|}
\hline & Admittance & History value \\
\hline Inductor & $G=\frac{\Delta t}{L}$ & $h(t)=G v(t)+h(t-\Delta t)$ \\
\hline Capacitor & $G=\frac{C}{\Delta t}$ & $h(t)=G v(t)$ \\
\hline
\end{tabular}

In EMT, to improve the simulation speed, we can sacrifice accuracy by applying one step delay method. In EMT type computer program, control systems are represented in high-level blocks. An example from EMT is shown in Figure. 2.3(a). 
Generally, the feedback loops $F(u)$ and $F(s)$ are non-linear. Therefore the whole system is non-linear. A simple and currently used method is to insert one time-step delay to break the feedback loop, if non-linear blocks exist [15]. As shown in Figure. 2.3(b), the feedback loop can use history value of output which is already known, there is no necessary to solve a equation set. This method provides a non-simultaneous solution for a nonlinear system, but it may lead to instabilities and end up in a wrong region. In some cases, it may speed up computation due to the reduction of iteration. In the most cases, this method imposes error and limitation of the term of accuracy, one step delay method should not bec $\mathrm{F}(\mathrm{n}) \mathrm{red}$ as a reliable method.

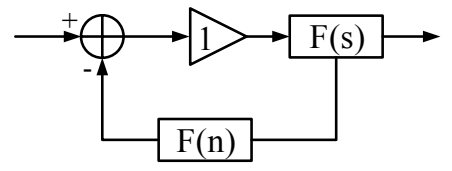

(a) Original

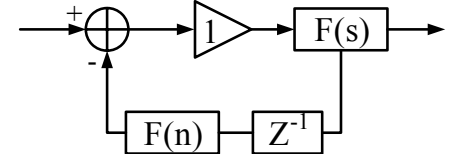

(b) One time-step delay inserted

Figure 2.3: Block diagram of control system in EMT

\subsubsection{Hybrid Simulation of Power System}

In the last decade, the hybrid simulation method had been proposed and developed to overcome the speed issues of EMT and accuracy issues of TS. The combination of TS and EMT was first proposed by Heffernan in 1981 [16. Two different simulators were integrated through HVDC link. Some further studies had been done in 17 19. The delightful idea of these techniques is to inherit the merits of both simulators and discard unnecessary data [20]. Some parts of the system run in TS simulator and the remaining parts run in EMT simulator. Hence, the computation speed is significantly increased and the level of accurate is partially preserved. Some details is lost in the 
TS simulator due to the phasor modelling method.

As a recently developed method, a hybrid simulator has following three categories issues: interface of two simulators, exchanging data and development of equivalent networks. The data format in TS and EMT simulators is different (frequency domain vs time domain). Therefore, a converter is required to convert data into suitable forms. In addition, time-step in each simulator is extremely different, and a interface protocol is necessary to coordinate data exchange between two simulators. It is to be noted that, the performance of hybrid simulator is based on the modelling accuracy of the TS and EMT simulator.

In this section, a review of various terms and definitions are reviewed firstly. Then, the application of hybrid simulator is presented.

\section{Classification of Hybrid Simulation}

In a hybrid simulator, TS and EMT run separately. An overview of the hybrid simulator is shown in Figure. 2.4. Each blocks will be explained in details in following section.

- Detailed System: It comprises the parts that required detailed modelling in the system. It can also be called instantaneous network 21] or EMT subsystem 22]. The detailed system is in the domain of EMT simulator. The typical time-step is in $\mu$ s range. Since the time-step is so small, in some case, an iterative solution can be avoided to save CPU time [23].

- External System: Other parts of power system are included in external system, which needs to be modelled in system level. It can also be called electromechanical transient network 24]. The external system is in the domain of TS simulator. Traditionally, the time-step for numerical integration is around 10ms. 
The external system is usually modelled as a set of algebraic equations. The variables are usually represented in phasor domain.

- Interface [25]: Buses between detailed systems and external systems are interface. They are used to exchange data and coordinate simulation order. How to exchange data efficiently and correctly is also a research direction in hybrid simulation field. Meanwhile, an interface is also responsible for data type converting. To connect these two different simulators, two data converters are necessary: phasor to waveform and waveform to phasor.

\section{Equivalent Circuit Representation}

Since the network modelling of two simulator is totally different, state variables must be transferred from one simulator to the other one. Thus, each subsystem needs a true picture which can reflect the characteristics correctly. The performance of hybrid simulator is directly affected by the accuracy of equivalent circuits.

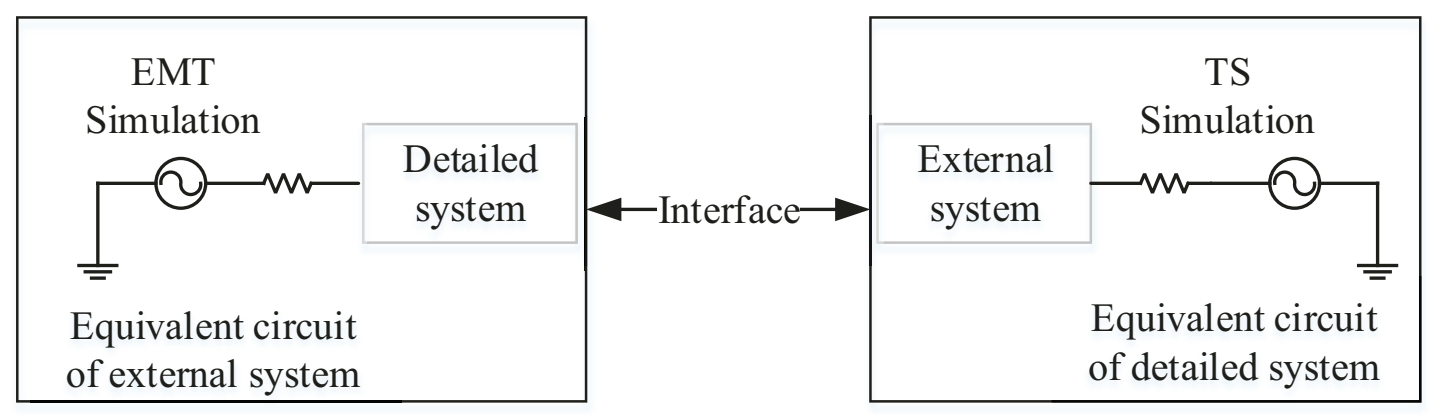

Figure 2.4: Overview of hybrid simulator

\section{Equivalent Circuit of External Subsystem in EMT}

The most basic method to obtain equivalent circuit is a fundamental frequency Norton equivalent [16]. One RLC shunt-connected branch with a current source is derived 
on power frequency. The impedance of external system is constant, therefore RLC value remains constant. The current source needs updating at the end of each timestep. If some components in external system change during simulation, like switch or fault, the RLC value also need updating [18. A more accurate method is using the frequency dependent equivalent network (FDEN), therefore whole range of frequency can be covered, even when high order harmonic distortions happen [26]. In [27], a detailed discussion about how to develop appropriate equivalent circuit based on admittance matrix is presented.

\section{Equivalent Circuit of Detailed System in TS}

In the last section, only current source modelling method has been used for external subsystem. However, detailed system can be modelled by not only current source but also other derived quantities like active/reactive power load or equivalent impedance [16]. These methods all require fundamental frequency of voltage and current at the interface bus. Thus, curve fitting or Fourier transformation are used to extract fundamental frequency. We can also assume the fundamental frequency is always $60 \mathrm{~Hz}$ but the accuracy is sacrificed.

\section{Interface and Interface protocol}

Since TS and EMT in hybrid simulation run in different time-step, interface protocol is necessary to coordinate working preemptive and exchange data. The time-step in TS should be integer multiplication of time-step in EMT. Otherwise, overrun will happen. One simulator has to stop and wait for data from the other simulator. Several protocols have been developed recently, serial protocol [28], 29] and parallel

protocol [26]. Some articles also give an automatic algorithm which can decide the type of protocol to be used during simulation [30,31]. In this section, three different 
protocols will be reviewed.

\section{Serial Protocol}

In a serial protocol, only one of the two simulators runs at the same time, while the other one keeps idle. Due to the existing of synchronous machine, TS simulator needs iterative method to solve nonlinear equations [32]. EMT simulator runs in a very small time-step, in the majority time, linear approximation can give accurate result as well. Figure. 2.5 shows the conventional form of a serial protocol. Time-step in $\operatorname{TS}(\Delta t)$ is $\mathrm{M}$ times of time-step in $\operatorname{EMT}\left(\Delta t^{\prime}\right)$.

$$
\Delta t=M \Delta t^{\prime}
$$

In Figure. 2.5, the upper line represents TS simulator and the bottom line represents EMT simulator. Step (1): Equivalent circuit in EMT simulator is updated by the quantities from TS simulator at $t=t[n]$. Step (2): EMT simulator proceeds to $t=t[k+M]$ and calculates positive sequence. Step (3): Current injection at time $t[n]$

can be calculated. Step (4): TS simulator can proceed to $t=t[n+1]$. Finally, step 2 to 4 are repeated until t equals required time. It is obviously that serial protocol cannot handle high speed simulation very well.

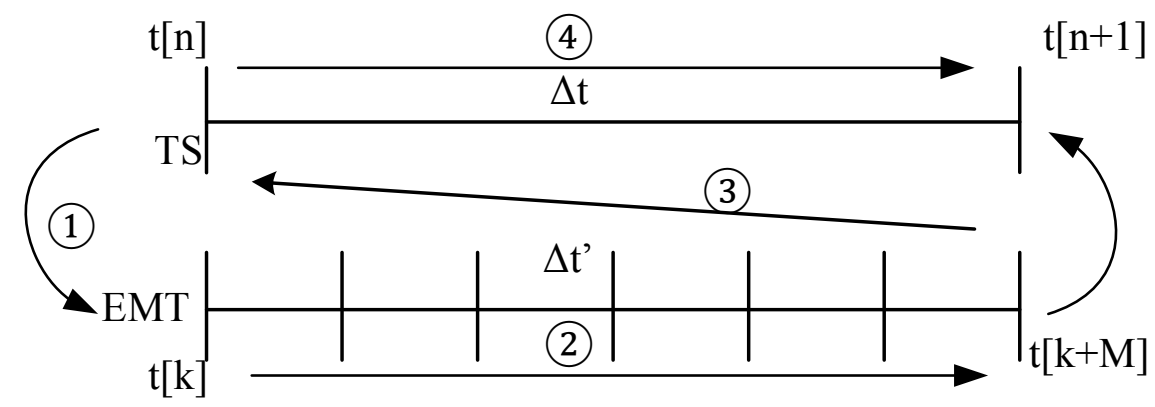

Figure 2.5: Serial interaction protocol of hybrid simulator 


\section{Parallel Protocol}

In a parallel protocol, two simulators can run simultaneously. This proposal overcomes the drawback in serial protocol, which can increase simulation speed as high as possible. The operation of a parallel protocol is shown in Figure. 2.6. Step (1): equivalent circuit in EMT simulator is updated by the quantities from TS simulator at $t=t[n]$. Step (2): Using the variables from TS simulator, EMT simulator proceeds to $t=t[k+M]$. Step (3): TS and EMT simulators exchange data through interface. Step (4): Both simulators run at the same time. TS proceeds to $t=t[n+1]$ and EMT proceeds to $t=t[k+2 M]$. Finally, step (3) and (4) are repeated until t equals required time. During the simulation, TS lags EMT for one time-step all the time. Therefore, the TS simulator uses old data to calculate value at the present time. When the transient change is large, parallel protocol may lead to instability and inaccuracy. Similar to One Step Delay method, the performance of parallel protocol depends on the time-step of EMT and the characteristic of the whole system.

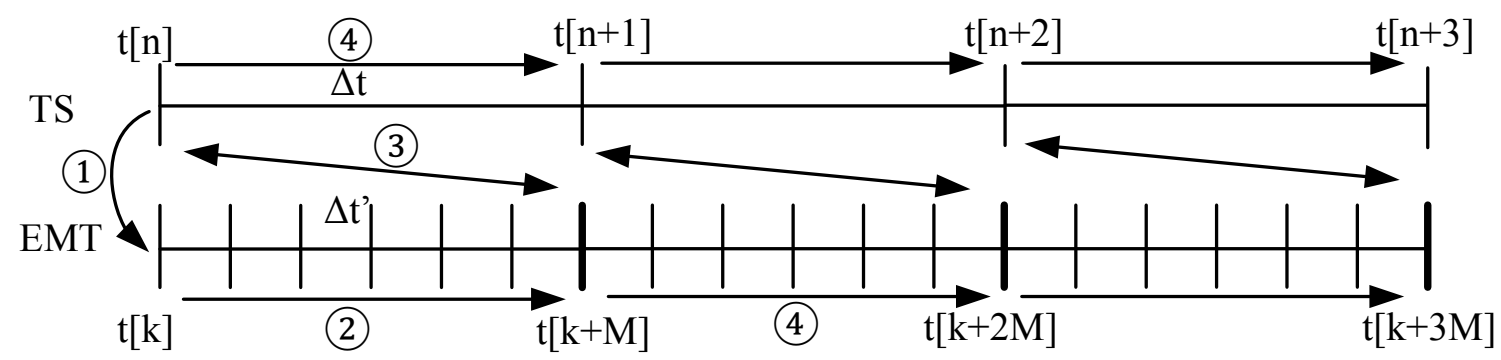

Figure 2.6: Parallel interaction protocol of hybrid simulator

\section{Combined Protocol}

Recently, some researchers developed a new interface protocol which combines serial and parallel protocol. In the majority time, hybrid simulator runs in parallel and 
in high speed. If disturbance happens, or the transient change is large, the interface protocol switches to serial protocol, which can provide more accurate simulation result [31]. Generally, two variables can be considered as criterions. One is the change of magnitude, the other is the rate of change. In principle, power, voltage and current are all able to detect the transient change. To reduce the communication load, current through interface bus is chosen as detection variable [30].

\subsection{Numerical Example}

The hybrid simulation method presented in the previous section has been validated on a practical system benchmark. The test benchmark used in this test, as shown in Figure. B.1, is the IEEE 9 bus system. Detailed parameters and modelling can be found in 33. This benchmark has a total of nine power buses and three synchronous machines. The bus 5 and its connected load are modelled in EMT simulator as a detailed system. The remaining system is modelled in the TS simulator as an external system. It is noted that the Thevenin equivalent circuit is used in this example. The equivalent admittance is $z=6.7294+57.14 j$, and the magnitude and phase are updated every time-step once the simulation starts. Block diagram of hybrid simulator is shown in Figure. 2.7. The TS simulation time-step is 5ms

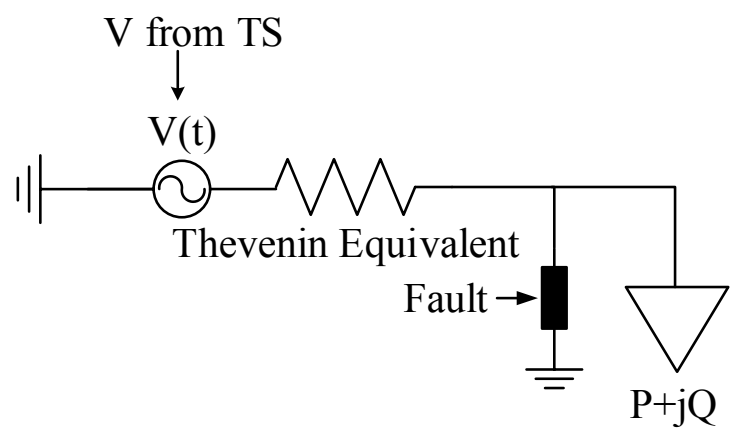

Figure 2.7: Detailed system along with the external system equivalent 
and the EMT simulation time-step is 5us. A three phase fault is applied at bus 5 at $0.6 \mathrm{~s}$ and cleared after 0.05s. The transient response is shown in Figure. 2.8. Blue line is obtained from hybrid simulation and red dashed line is obtained by EMT simulation (PSCAD) as reference. Both simulators start from zero initial condition. EMT simulator gives more details when fault happens. Although hybrid simulator runs faster than EMT simulator, the simulation result is not ideal.

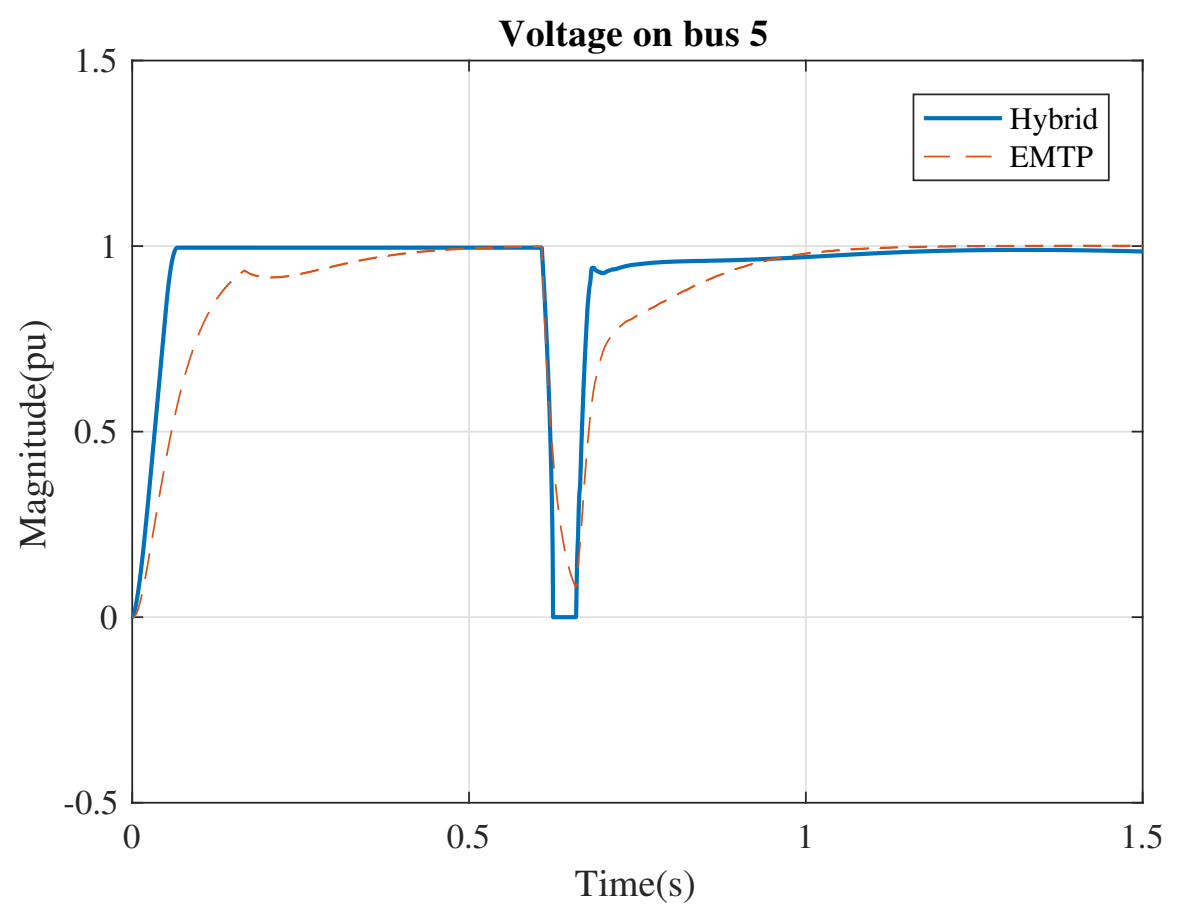

Figure 2.8: Comparison between hybrid simulator and EMT simulator (Voltage)

\subsection{Recent Developments}

Hybrid simulation has developed a lot in the recent five years. 34 proposed a new equivalent circuit called dynamically updated Thevenin- Norton equivalents, which is quite good for convergence. Although the simulation speed is improved, it can only 


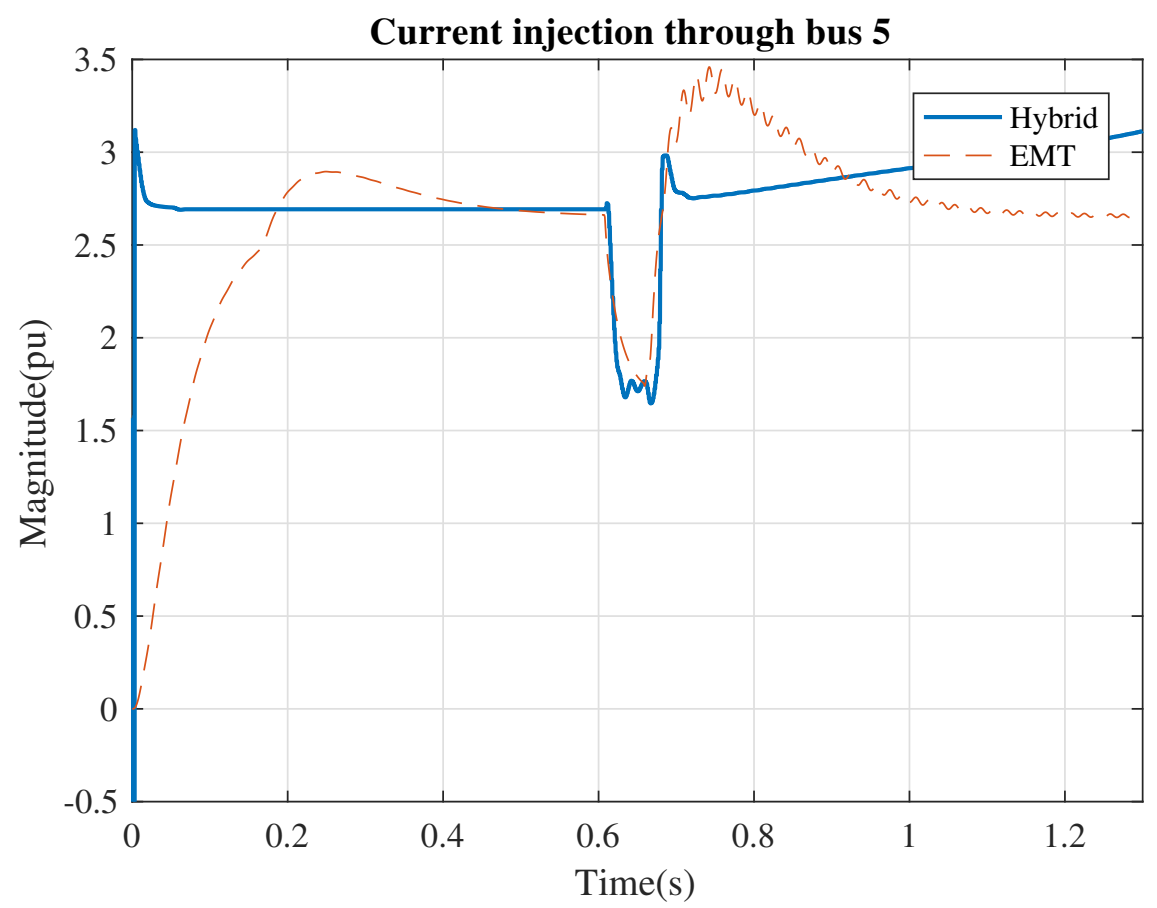

Figure 2.9: Comparison between hybrid simulator and EMT simulator (Current)

simulate sinusoidal and balanced power system. Some researchers focus on developing new equivalent circuit to improve the accuracy [35, 36].

Dewu developed a novel dynamic phasor based model for hybrid simulation [37. Waveform distortion is included in the TS simulator now. This method can promote the accuracy improvement. Meanwhile, a new block called dynamic phasor based interface model (DPIM) is introduced. More preparation should be done before simulation, which means this method is not general.

The authors of 38 proposed a new method to speed up convergence. The average iteration number reduces from six to three. However, three times of iteration is the average iterations in the new proposed method of this thesis.

Other papers like $26,30,39,40$ focus on developing the protocol, either reducing the exchange rate or developing a new protocol. Other papers describe a novel implicit coupling method that can combine TS and EMT in equation level instead of physics 
level 41, 42. This new coupling method can make TS and EMT run simultaneously.

Parallel calculation is another hot topic in power system simulation. [43] shows the parallel computing of power distribution system. In this paper, appliances are separated into different types and simulated in different processors. When the number of appliances increases, the simulator is no longer feasible. But the software used in this paper is shared memory. No message passing interface is needed. Alvarado proposed a method called W-Matrix [44]. W-matrix algorithm converts $A x=b$ into the multiplication of matrices and calculates the multiplication in parallel. Another parallel algorithm called Parallel General Norton with Multi-port Equivalent(PGNME) was proposed in 45]. PGNME partitions the large linear power system into several small one and builds Norton equivalent circuit for each port. The simulator is written in $\mathrm{C}++$ to achieve the fastest execution. However, this method only works in transient stability simulator not in electromagnetic transient simulator. Another paper uses hierarchical block bordered diagonal form (BBDF) algorithm for power system simulation [46]. BBDF method aims to generate a matrix which is suitable for parallel calculation based on the transient stability. The partitioning method in this paper has dynamic partitioning method which improves the precision and robustness of the algorithm. 47] improves the BBDF method by introducing predictor-corrector interior point method. The main algorithm is the same as BBDF and partitioning method is based on the physical location.

Based on these recent researches, we can find that hybrid simulation has some obvious drawbacks. Equivalent circuit has to be done before simulation and there are no general methods to develop an accurate model. Protocol is another obstacle that affects performance of hybrid simulation. Although parallel calculation is applied in the power system, the majority methods still need equivalent circuit and the partitioning method is not efficient. Therefore, a new algorithm is proposed, that no 
approximation and no protocol are needed. 


\section{Chapter 3}

\section{Power System Components Modelling}

To perform power system dynamic simulations and analyze the power system stability, we need to model the power system correctly and efficiently. In this chapter, some common components in the power system are introduced. Before modelling the power system, a general nonlinear system is given. Synchronous machines, as being the principal sources in power systems, are first explored. Furthermore, a wind turbine is explained in detail including the modelling and control theory. Finally, the grid is modelled in three phase and time domain by the Modified Nodal Analysis(MNA) method, instead of using the phasor model. The traditional MNA formulation method only contains electrical signal. However, power system contains synchronous machine which needs physical modelling. The MNA formulation of power system contains some physical variables like rotating speed, rotating angle, and magnetic flux. The machine is modelled as an element and added to a specific power bus. By this modified MNA formulation, the power system is modelled exactly the same as its physical model. 


\subsection{Formulation of System Equations}

In general, a nonlinear system can be described by (3.1). $n$ is the number of unknown variables.

$$
\begin{gathered}
\boldsymbol{C} \frac{d \boldsymbol{x}(t)}{d t}+\boldsymbol{G} x(t)+\boldsymbol{F}(\boldsymbol{x}(t))=\boldsymbol{b}(t) \\
\boldsymbol{y}(t)=\boldsymbol{d}^{T} \boldsymbol{x}(t)
\end{gathered}
$$

where,

$\boldsymbol{x} \in \mathbb{R}^{n}$ is the vector of unknown variables,

$\boldsymbol{G} \in \mathbb{R}^{n \times n}$ matrix denotes the non-memory elements and $\boldsymbol{C} \in \mathbb{R}^{n \times n}$ matrix denotes the memory elements, $\mathrm{n}$ is the size of matrix which depends on the size of the whole system,

$\boldsymbol{F}(\boldsymbol{x}(t)) \in \mathbb{R}^{n}$ is the vector containing some non-linear items in the system,

$\boldsymbol{b} \in \mathbb{R}^{n}$ is the excitation or the input of the system,

$\boldsymbol{y} \in \mathbb{R}^{n}$ is the vector of output and

$\boldsymbol{d} \in \mathbb{R}^{n_{\text {out }} \times n}$ is the selection matrix.

After applying numerical integration methods, such as the Trapezoidal rule to (3.1). The nonlinear differential equations can be transformed into difference equations. We can obtain:

$$
\begin{aligned}
\left(\boldsymbol{G}+\frac{\boldsymbol{C}}{h}\right) \boldsymbol{x}(t+h)= & \boldsymbol{C} x(t)+\frac{1}{2}(\boldsymbol{F}(x(t))-\boldsymbol{G} \boldsymbol{x}(t)-\boldsymbol{D} \\
& +\boldsymbol{F}(\boldsymbol{x}(t+h))-\boldsymbol{G} \boldsymbol{x}(t-h)-\boldsymbol{D})
\end{aligned}
$$

where $h$ is a fixed time-step for this transient analysis 48]. Eq. (3.2) can be written in a more general form: 


$$
\boldsymbol{A}(h, \boldsymbol{G}, \boldsymbol{C}) \boldsymbol{x}(n+1)+\boldsymbol{F}(\boldsymbol{x}(n+1))-\boldsymbol{K}(\boldsymbol{x}(n))=0
$$

where,

$h$ is the time-step in the simulator,

$\boldsymbol{A}(h, \boldsymbol{G}, \boldsymbol{C}) \boldsymbol{x}(n+1), \boldsymbol{K}(\boldsymbol{x}(n))$ are functions of $h, \boldsymbol{G}, \boldsymbol{C}, \boldsymbol{x}(n)$. Different functions are applied based on different integration method,

$\boldsymbol{x}(n)$ is the vector of the variables in system at the $\mathrm{n}$-th time point.

Detailed modelling process is discussed in the following section. After that, the corresponding system equations can be obtained easily. Traditionally, the solution of (3.3) requires NR iteration and direct matrix factorization. The solution is given by:

$$
\Delta \boldsymbol{x}=\boldsymbol{J}^{-1} \phi(x)
$$

where,

$\boldsymbol{J}=\boldsymbol{A}(h, \boldsymbol{G}, \boldsymbol{C})+\partial \boldsymbol{F} / \partial \boldsymbol{x}$ is the Jacobian matrix,

$\boldsymbol{\phi}=\boldsymbol{A}(h, \boldsymbol{G}, \boldsymbol{C}) \boldsymbol{x}(n+1)+\boldsymbol{F}(\boldsymbol{x}(n+1))-\boldsymbol{K}(\boldsymbol{x}(n))$,

$\Delta \boldsymbol{x}$ is the change of variables in each iterations.

\subsection{Park and Clark Transformation}

To simplify analysis and reduce the number of equations in (3.1), the direct quadrature zero transformation as a tensor that rotates frame of a three-phase vector is used. The $D Q$ transform is based on the Clark transform and the Park transform [49], which can convert time-varying values to constant values. The transformation between $a b c$ 
and $d q$ is shown as follows:

$$
\begin{gathered}
{\left[\begin{array}{l}
V_{d} \\
V_{q}
\end{array}\right]=\frac{2}{3}\left[\begin{array}{ccc}
\cos (\theta) & \cos \left(\theta-\frac{2}{3} \pi\right) & \cos \left(\theta+\frac{2}{3} \pi\right) \\
-\sin (\theta) & -\sin \left(\theta-\frac{2}{3} \pi\right) & -\sin \left(\theta+\frac{2}{3} \pi\right)
\end{array}\right]\left[\begin{array}{l}
V_{a} \\
V_{b} \\
V_{c}
\end{array}\right]} \\
{\left[\begin{array}{l}
V_{a} \\
V_{b} \\
V_{c}
\end{array}\right]=\left[\begin{array}{rr}
\cos (\theta) & -\sin (\theta) \\
\cos \left(\theta-\frac{2}{3} \pi\right) & -\sin \left(\theta-\frac{2}{3} \pi\right) \\
\cos \left(\theta+\frac{2}{3} \pi\right) & -\sin \left(\theta+\frac{2}{3} \pi\right)
\end{array}\right]\left[\begin{array}{l}
V_{d} \\
V_{q}
\end{array}\right]}
\end{gathered}
$$

The relationship between $a b c$ frame and $d q$ frame is also illustrated in Figure. 3.1. The angle $\theta$ is the rotating angle of the machine or the angle of three phase voltage. In grids, it can be obtained by a three-phase Phase Lock Loop(PLL). After analysis

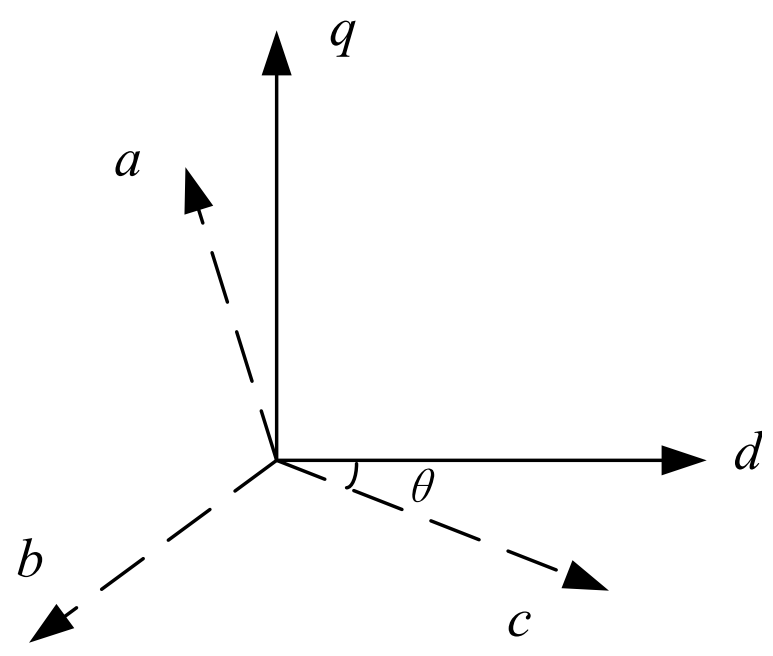

Figure 3.1: $a b c$ and $d q$ frame 
and simulation, to present the result, (3.6) is used to convert constant value back to three phase sine waves. The calculation of this part can be finished after simulation.

\subsection{Modelling of Synchronous Generator}

Generator modelling is carried out by using a fourth-order model. Higher order model is available in [13]. This model, we have following assumptions:

- Magnetic hysteresis is negligible

- Magnetic saturation effects are negligible

- The windings' resistances are negligible

Eq. (3.7) to (3.10) are used to model the generator. Eq.(3.7) and Eq.(3.8) are electrical parts and Eq. 3.9 and Eq. 3.10) are mechanical parts. $E_{d}$ and $I_{d}$ represent the $d$ axis transient voltage and armature current. $E_{q}$ and $I_{q}$ represent the $q$-axis transient voltage and armature current. $\delta$ and $\omega$ are the rotor angular position and angular speed, respectively.

$$
\begin{gathered}
E_{q}^{\prime}=\frac{1}{T_{d 0}^{\prime}}\left(-E_{q}-\left(x_{d}-x_{d}^{\prime}\right) I_{d}+E_{f d}\right) \\
E_{d}^{\prime}=\frac{1}{T_{q 0}^{\prime}}\left(-E_{d}+\left(x_{q}-x_{q}^{\prime}\right) I_{q}\right) \\
\delta^{\prime}=\omega \\
\omega^{\prime}=\frac{1}{H}\left(T_{m}-\left(\phi_{d} I_{q}-\phi_{q} I_{d}\right)-D \omega\right)
\end{gathered}
$$

where $x_{d}, x_{d}^{\prime}$ are $d$-axis synchronous reactance and transient reactance, $T_{d 0}^{\prime}$ is $d$-axis

open-circuit time constant, $T_{q 0}^{\prime}$ is $q$-axis open-circuit time constant, $E_{f d}$ is field voltage, $H$ is inertia constant and $D$ is damping coefficient. 


\subsubsection{IEEE Type 1 Execitor Model}

The main purpose of an exciter is keeping the output voltage as stable as possible.

$E_{f}$ is the voltage applied to the exciter system. In this project, IEEE type 1 execitor is used [50], which is shown in Figure. 3.2

$$
\begin{gathered}
E_{s}=V_{r e f}-\frac{V_{c}}{1+s T_{r}}-\frac{s K_{f}}{1+s T_{f}} E_{f} \\
E_{f}=E_{s} * \frac{K_{a}}{1+s T_{a}} * \frac{1}{K_{e}+s T_{e}}
\end{gathered}
$$

where,

$T_{r}$ is transducer time constant,

$T_{a}$ is AVR time constant,

$T_{e}$ is exciter time constant,

$T_{f}$ is field voltage feedback time constant,

$K_{a}$ is AVR gain,

$K_{f}$ is field voltage feedback gain,

$S E$ is Saturation voltage point. 


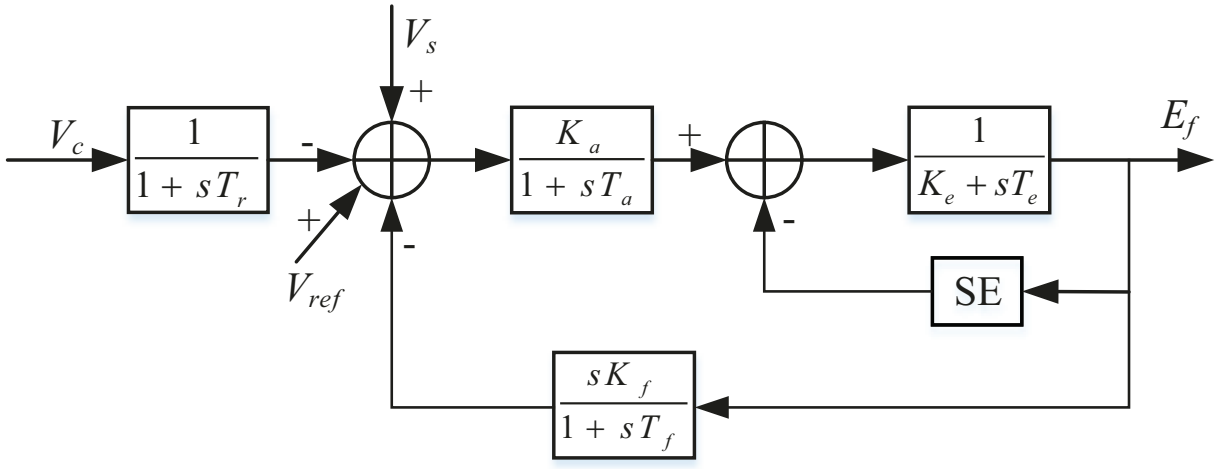

Figure 3.2: IEEE Type 1 exciter block diagram

\subsubsection{Governor Model}

Governor aims to keep the rotating speed at a constant value. The output frequency is related to the rotating speed. Therefore, the rotating speed should be kept at $2 \pi f$. The structure is shown in Figure. 3.3. The specific response time of the governor is 3 - 5s. Therefore, only long duration simulations will contain this subsystem.

$$
\begin{gathered}
P_{m}^{\prime}=-\frac{1}{T_{t}} P_{m}-\frac{1}{T_{t}} P_{g}+\frac{1}{T_{t}} \omega_{r e f} \\
P_{g}^{\prime}=-\frac{K_{g}}{T_{g}} \omega-\frac{1}{T_{g}} P_{g}+\frac{K_{g}}{T_{g}} P_{r e f}
\end{gathered}
$$

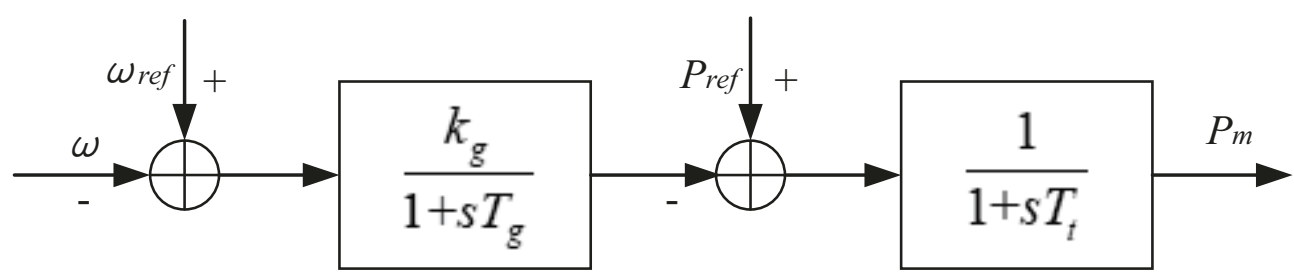

Figure 3.3: Governor block diagram 


\subsubsection{DQ - abc interface}

The frame in the machine is $d q$ frame, which simplifies the analysis process a lot. However, in the grid, to obtain detailed three-phase simulation results, the modelling must be finished in $a b c$ frame. The following equations represent the interfaces between $d q$ frame and $a b c$ frame based on section. 3.2 .

$$
\begin{gathered}
V_{d q}=\boldsymbol{K}_{C P} V_{a b c} \\
I_{a b c}=\boldsymbol{K}_{C P}^{-1} I_{d q}
\end{gathered}
$$

After making the transformation, the varying waveform (left part of Figure. 3.4) will be converted into two constant values (right part of Figure. 3.4). After the analysis and calculation, they are transformed back to the normal waveform.
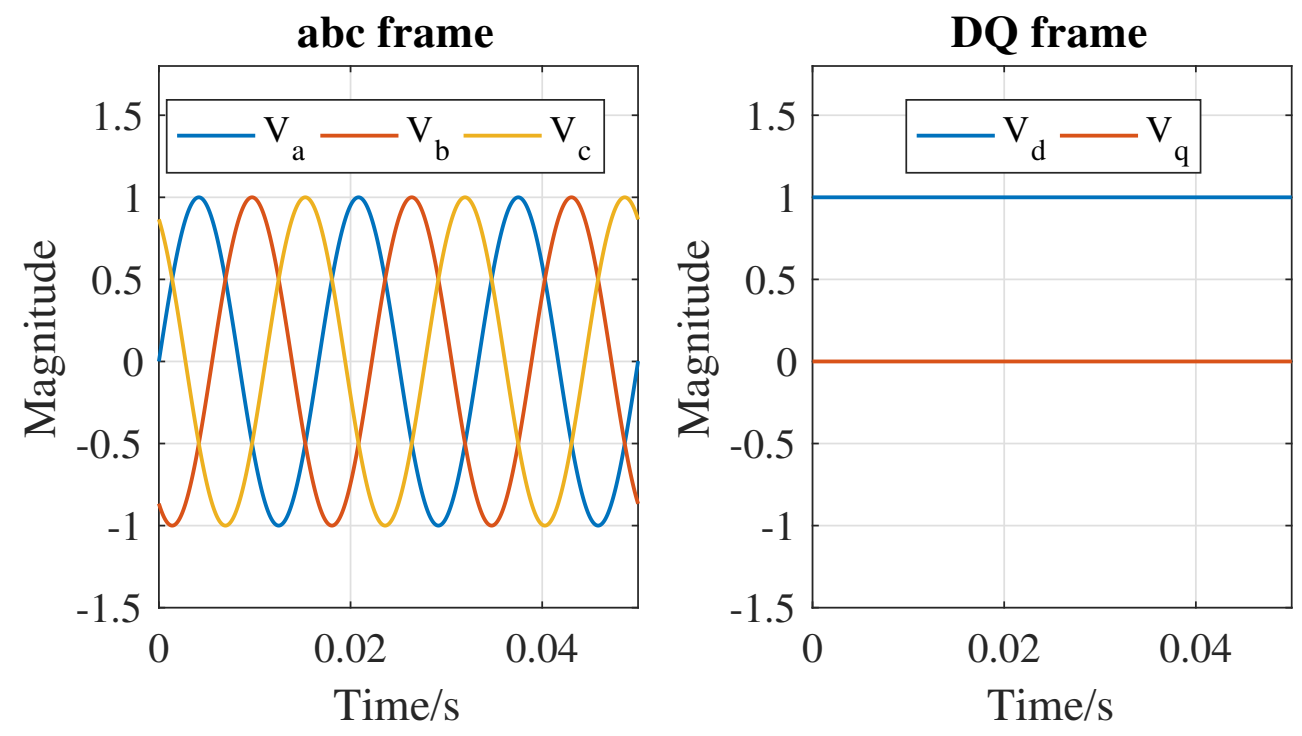

Figure 3.4: Three phase voltage in $a b c$ and $d q$ frame 


\subsection{Modelling of Wind Energy Conversion System}

Wind power is the fastest-growing source of electricity and has attracted the attention of the world. The PMSG, as one of the most important parts in WECS is worth exploring. As shown in Figure. 3.5, WECS mainly consists of a wind turbine, one generator and one back to back converter. Each part will be modelled in detail.

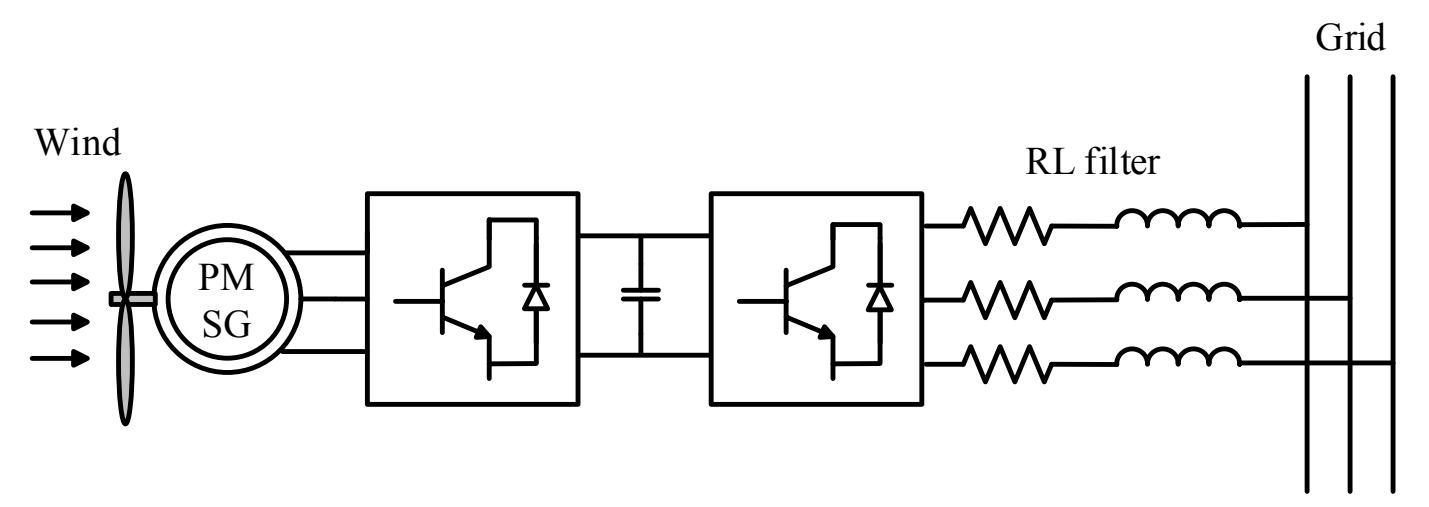

Figure 3.5: Block diagram of WECS

Back to back converter

\subsubsection{Aerodynamic and Mechanical Systems}

Wind turbine is the device that transffers the kinetic energy from wind into thechanical

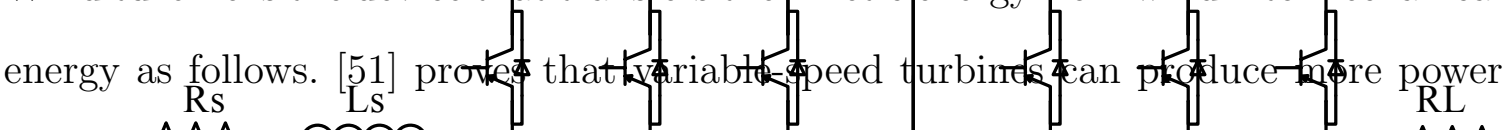
comparefly the constant on e. However, power converters and their contris system LL are necessary to keep the fundamental frequency aad powe factor be const)

The power coefficient of the turbine $\left(C_{p}\right)$ is introduced here. $C_{p}$ is the ratio of actual electric power produced by a wind turbine divided by the total wind power flowing 
into the turbine blades at specific wind speed. $\beta$ (pitch angle) is used to describe the angle of the turbine blade. Tip speed ratio $(\lambda)$ describes the ratio of tip speed of the blade and wind speed.

$$
\lambda=\frac{\omega_{m} R}{V_{\text {wind }}}
$$

The equation of power efficient is illustrated in 3.19 .

$$
C_{p}(\lambda, \beta)=C_{1}\left(\frac{C_{2}}{\lambda_{i}}-C_{3} \beta-C_{4}\right) \exp \left(\frac{-C_{5}}{\lambda_{i}}\right)+C_{6} \lambda
$$

where

$$
\frac{1}{\lambda_{i}}=\frac{1}{\lambda+0.08 \beta}-\frac{0.035}{\beta^{3}+1}
$$

and the coefficients $C_{1}$ to $C_{6}$ are: $C_{1}=0.5176, C_{2}=116, C_{3}=0.4, C_{4}=5, C_{5}=$ $21, C_{6}=0.0068$, respectively. According to the equation, the $C_{p}$ versus $\lambda$ can be seen in Figure. 3.6. The maximum coefficient happens when tip speed ratio equals to 8.1 and pitch angle equals to 0 degree. If the air density and blade area are invariable, $P_{w}$ only depends on $C_{p}$.

$$
P_{w \max }=0.5 \rho \pi r^{2} C_{p \max }\left(\frac{r \omega_{t}}{\lambda_{\text {opt }}}\right)=k_{\text {opt }} \omega_{t}^{3}
$$

To capture the maximum power from wind, a wind turbine should always work in this condition. Many maximum power point track (MPPT) methods are designed based on this principle. Recently, the direct driven PMSG is more popular due to its advantages, including efficient, reliability and no gearbox. The driven train is represented by one-mass model:

$$
\omega_{m}^{\prime}=\frac{1}{J}\left(T_{e}-T_{m}\right)
$$




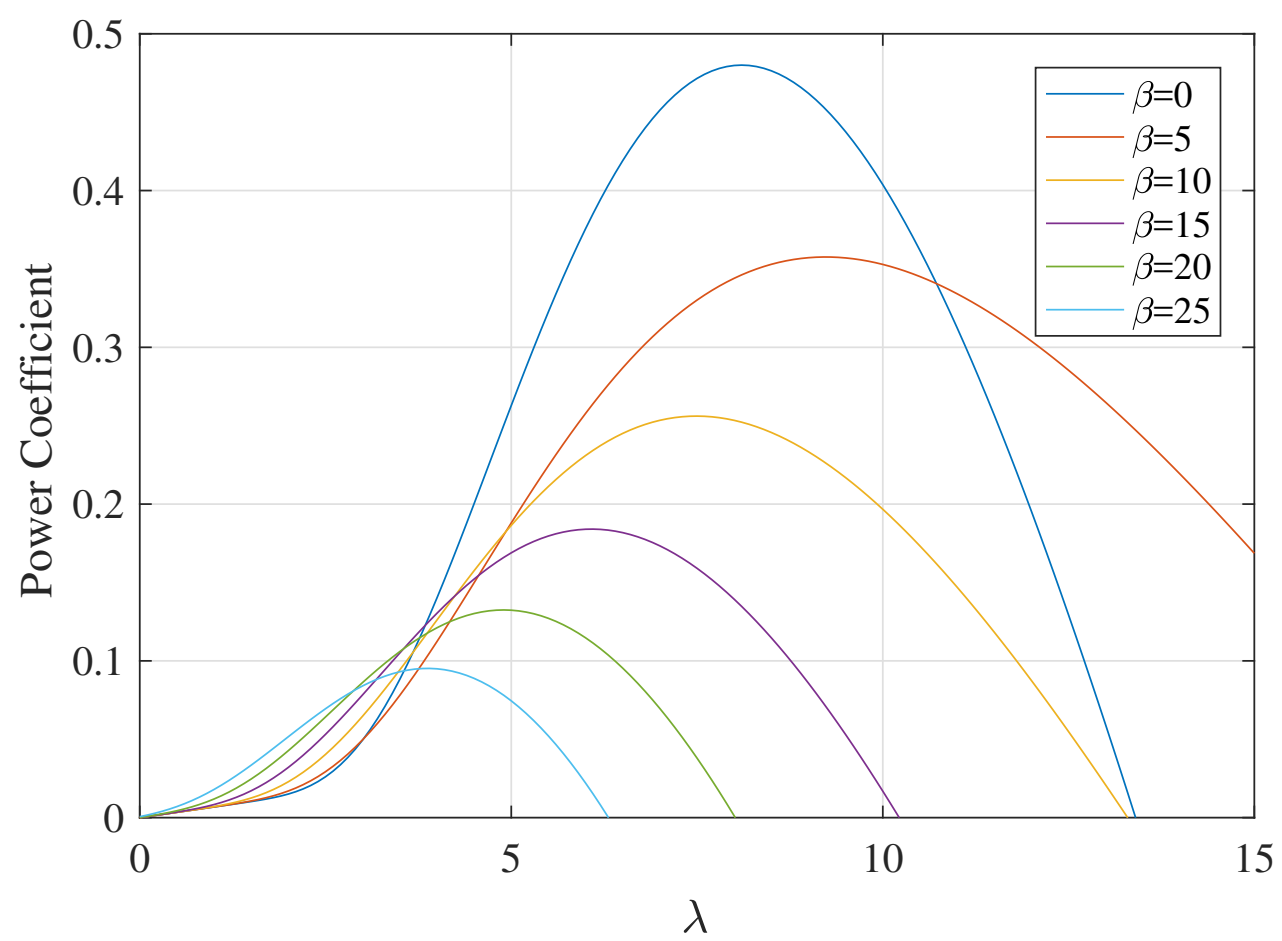

Figure 3.6: Turbine characteristic curves

where $\omega_{m}$ is the rotating speed of blade, $T_{m}$ is the wind turbine mechanical torque output, $T_{e}$ is electromagnetic torque of PMSG and $\mathrm{J}$ is the moment of inertia.

\subsubsection{PMSG}

Based on the transformation technique described in Section. 3.2 , the electromagnetic equations of a PMSG are described based on the $d q 0$ frame.

$$
\begin{gathered}
i_{d}^{\prime}=\frac{1}{L_{d}} V_{d}-\frac{R_{s}}{L_{d}} i_{d}+\frac{L_{q}}{L_{d}} N_{p} \omega_{m} i_{q} \\
i_{q}^{\prime}=\frac{1}{L_{q}} V_{q}-\frac{R_{s}}{L_{q}} i_{q}-\frac{L_{d}}{L_{q}} N_{p} \omega_{m} i_{d}-\frac{\psi N_{p} \omega_{m}}{L_{q}}
\end{gathered}
$$

where $L_{d}, L_{q}$ and $R_{s}$ are the generator inductances and resistance. $\omega_{m}$ is the angular speed of the rotor in the generator. $\psi$ is the flux and $N_{p}$ is the number of poles pair. 


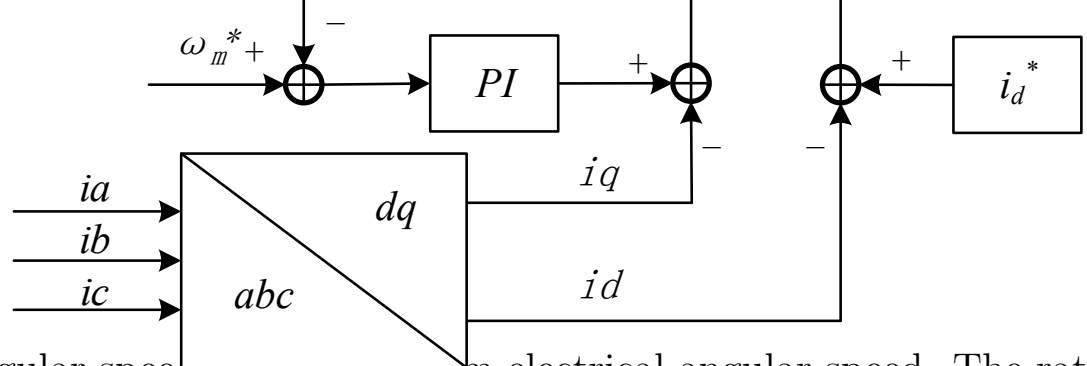

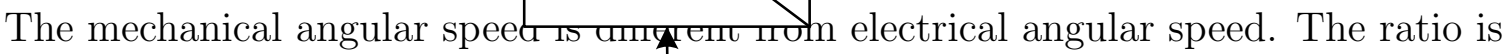
pair of poles $\left(N_{p}\right)$. Therefore, the ele $\theta$ trical angle of the machine is:

$$
\delta^{\prime}=\omega_{e}=N_{p} \omega_{m}
$$

The electromagnetic torque is expressed as:

$$
T_{e}=1.5 * N_{p}\left[\psi i_{q}-\left(L_{d}-L_{q}\right) i_{d} i_{q}\right]
$$

If $L_{d}$ equals $L_{q}$, the electromagnetic torque is proportional $i_{q}:$

$$
T_{e}=1.5 N_{p} \psi i_{q}
$$

\subsubsection{Generator Side Controller}

The control strategies applied to the generator side converter are illustrated in Figure. 3.7. The current from PMSG and the DC-link voltage work as input signals to the controllers. The generator side converter controls the rotating speed by controlling

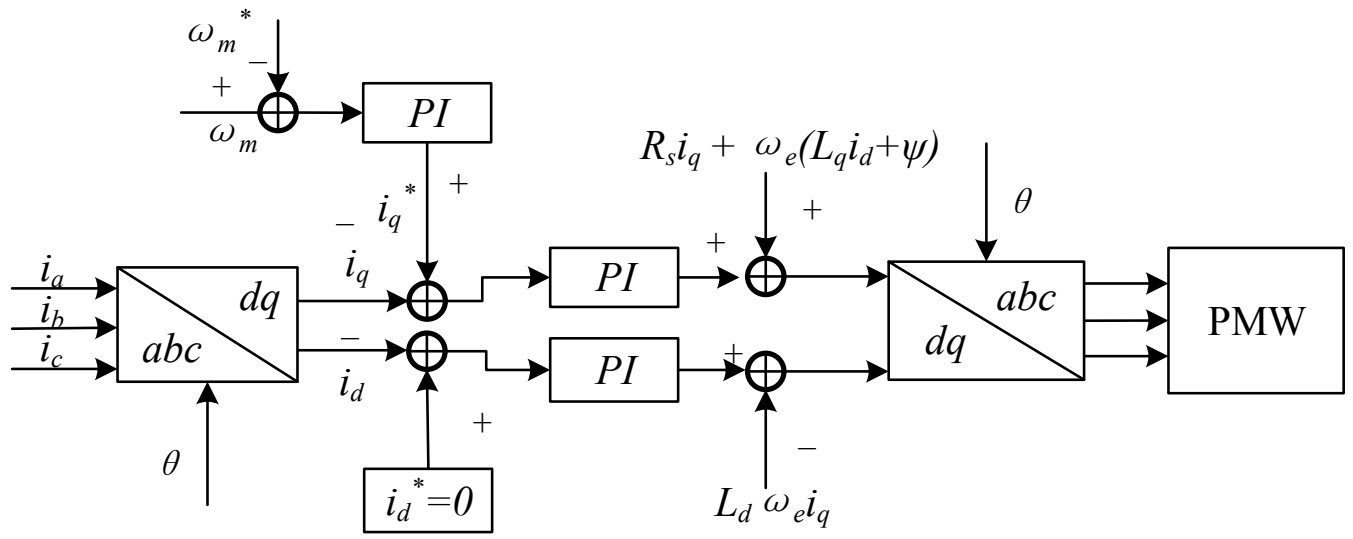

Figure 3.7: Control block for the generator side converter 
$i_{q} . i_{q}$ can affect the electromagnetic torque directly. If $T_{e}$ is smaller than $T_{m}$, the rotating speed will increase. Vice versa, if $T_{e}$ is larger than $T_{m}$, the rotating speed will decrease. The optimal rotating speed is $\frac{V_{w} \lambda}{R}$. Therefore, a PI controller is used to regulate the rotating speed:

$$
i_{q}^{*}=\left(K_{p m}+\frac{K_{i m}}{s}\right)\left(\frac{8.1}{R} V_{w i n d}-\omega_{m}\right)
$$

This controller can also be called Maximum Power Point Track(MPPT) control. With the help of this controller, a wind turbine can absorb the maximum power from wind. After that, two current control loops are employed. $i_{q}^{*}$ is obtained from MPPT, and a simple PI control is implemented after it. To reduce the power loss in rotor resistance and to obtain the highest efficiency, the $d$-axis current reference value is set to zero [52]. Before applying the signal to converters, we need to decouple these two variables. To decouple the $d$-axis and $q$-axis current, the compensation terms are added. Based on the voltage equation for the PMSG (3.23) and (3.24), for $d$-axis, the compensation is $-L_{d} N_{p} \omega_{m} i_{q}$. For the $q$-axism the compensation is $R_{s} i_{q}+N_{p} \omega_{m} L_{d} i_{d}+\psi N_{p} \omega_{m}$. In summary, machine side controllers are represented by 3.29) and 3.30.

$$
\begin{gathered}
V_{d}^{\prime}=\left(K_{p g}+\frac{K_{i g}}{s}\right)\left(-i_{d}\right)-L_{d} N_{p} \omega_{m} i_{q} \\
V_{q}^{\prime}=\left(K_{p g 2}+\frac{K_{i g 2}}{s}\right)\left(i_{q}^{*}-i_{q}\right)+R_{s} i_{q}+N_{p} \omega_{m} L_{d} i_{d}+\psi N_{p} \omega_{m}
\end{gathered}
$$

\subsubsection{Grid Side Controller}

Due to the existence of a back-to-back converter, the grid converter and generator converter will not affect each other. The aims of the grid side inverters are to deliver the energy from the PMSG to the grid and to achieve unity power factor [53]. At 


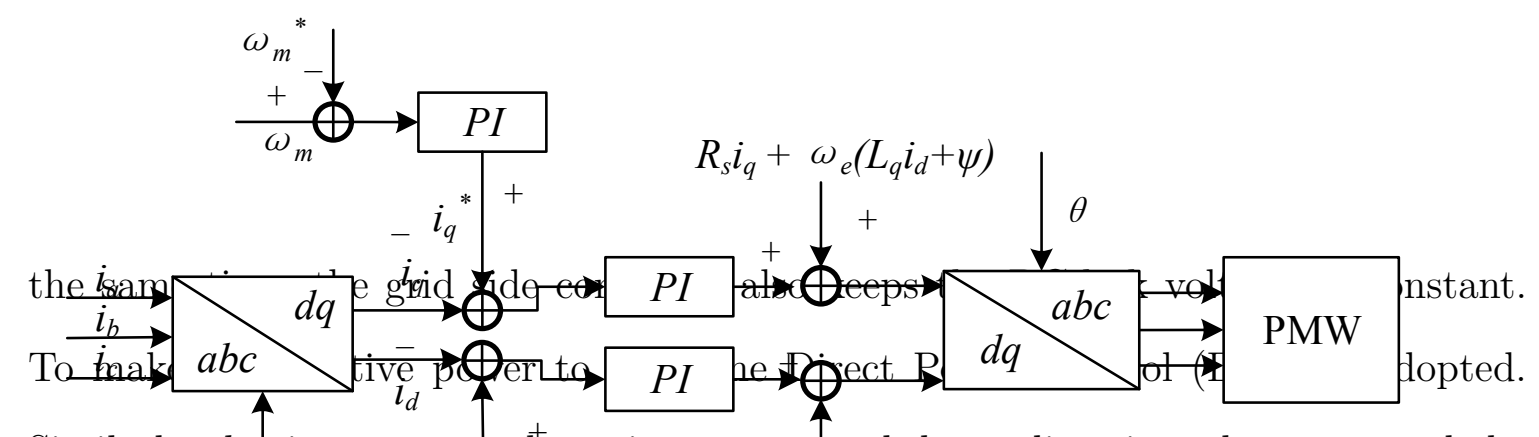

Similarly, $d_{-}$-axis current and ${ }^{+} q$-axis current heed decoupling, in order to control the

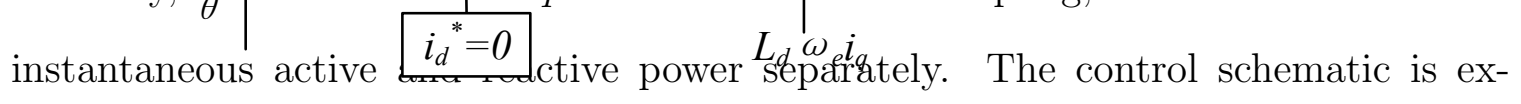
pressed in Figure. 3.8. There are two control loops. The outer loop is used to control the DC voltage, making it stable. The inner loops, active and reactive powers are controlled respectively by controlling $i_{d}$ and $i_{q}$. If the grid voltage is oriented on d-axis, the voltage on q-axis should be zero. Then, the active and reactive power can be represented by 3.31 and 3.32 .

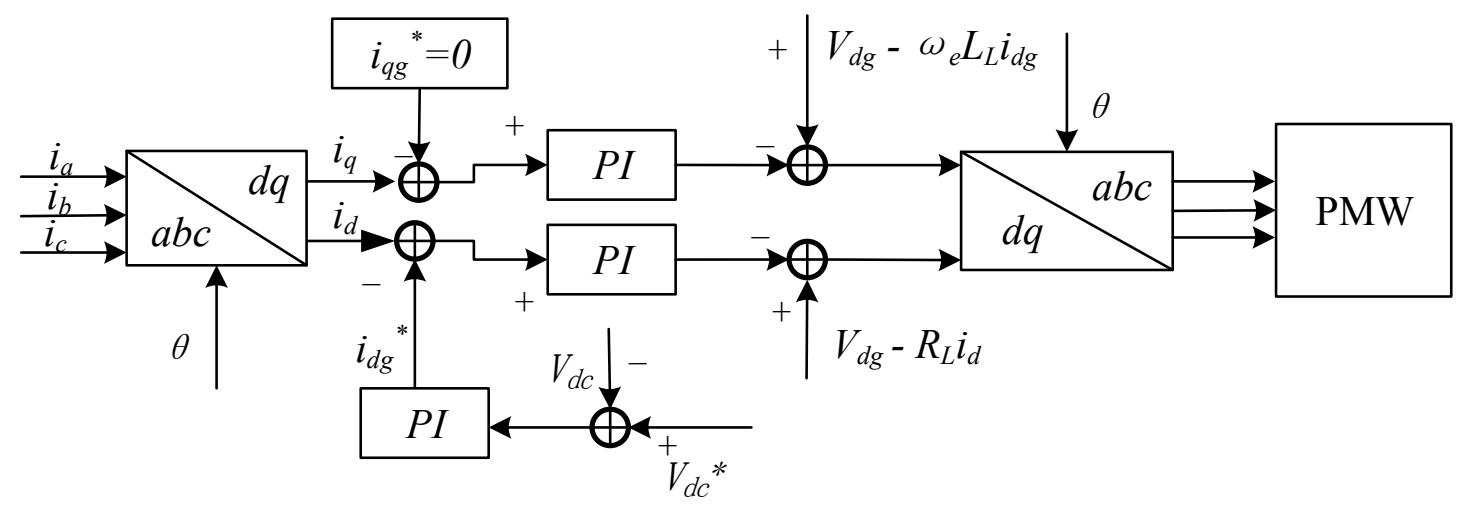

Figure 3.8: Control block for the grid side converter

The relationships between current and power are shown in 3.31 and 3.32). The $d$-axis current controls the active power whereas $q$-axis current controls the reactive power. The reference of $q$-axis grid current $i_{q g}^{*}$ is set as 0 to maintain unity power factor.

$$
\begin{aligned}
& P=1.5 V_{d g} i_{d g} \\
& Q=1.5 V_{d g} i_{q g}
\end{aligned}
$$

$d$ axis reference current $\left(i_{d g}^{*}\right)$ is dynamically decided by DC voltage. In summary, 
the grid side control schemes are described by (3.33) and 3.34).

$$
\begin{gathered}
V_{d 1}^{\prime}=-\left(K_{p s 2}+\frac{K_{i s 2}}{s}\right)\left[\left(K_{p d c}+\frac{K_{i d c}}{s}\right)\left(V_{d c r e f}-V_{d c}\right)-i_{d g}\right]+U_{d}+i_{q g} L_{L} \omega_{e}-R_{L} i_{d g} \\
V_{q 1}^{\prime}=\left(K_{p s}+\frac{K_{i s}}{s}\right) i_{q g}+U_{q}-R_{L} i_{q g}-i_{d g} L_{L} \omega_{e}
\end{gathered}
$$

\subsubsection{Modelling of AC-DC-AC Voltage Source Con- verter(VSC)}

A back to back VSC is usually used to link the direct drive generator and the utility

grid, as shown in Figure. 3.9. The response of the power electronic device is characterized by a high frequency phenomena. Due to the different objectives of different studies, a VSC can be modelled in different level. The topology of the back to back VSC comprises a double conversion from AC to DC and then DC to AC. The simplest model is the average model. The three controlled voltage sources replace the switching devices, as shown in Figure. 3.10. Correspondingly, the current is calculated by the power equation and the high frequency components are ignored. The internal loss of a VSC is also not calculated.

Another model is the detailed model, each power electronic component is approximated by an impedance. A small impedance for ON state and a huge impedance for OFF state. This model can inherit the characteristic of the high-frequency switching effect. Figure. 3.11 shows the simulation results of DC link voltage by using two different models. The detailed model gives more harmonic components, while the average model only gives the mean value. 


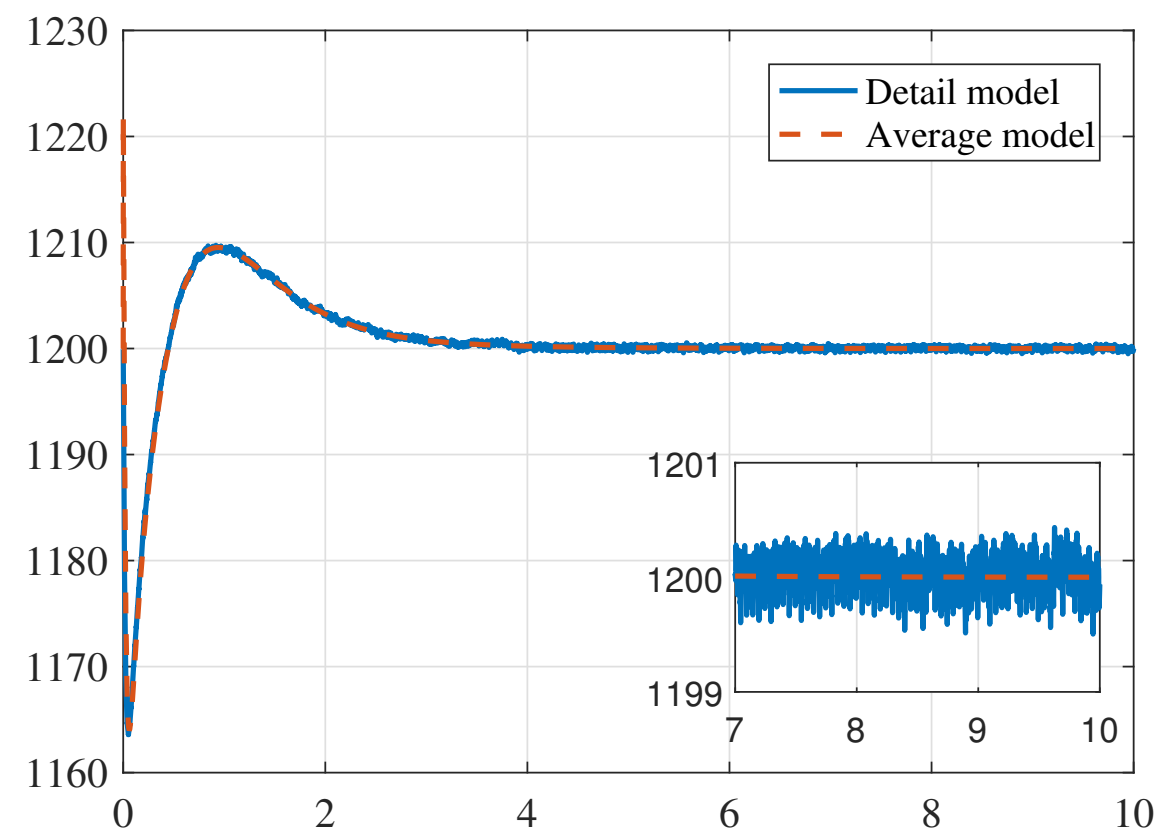

Figure 3.11: Comparison of average model and detailed model(DC link)

to power system simulation easily. When the transmission line is long (larger than $200 \mathrm{~km})$, correction is required to obtain accurate model [54].

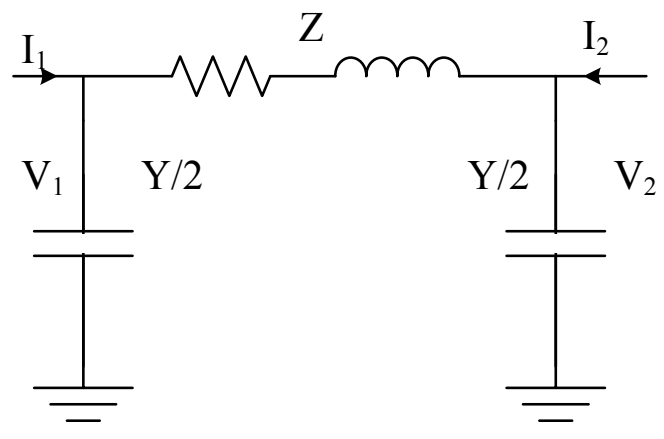

Figure 3.12: Transmission line $\pi$ model 


\subsection{Loads}

In this thesis, loads are modelled by constant power and linear loads. Therefore, the model is consisting of shunt RLC circuits. Resistance consumes the active power while an inductor and a capacitor consume the reactive power. Similar to the transmission line, the terminal of each load corresponds a bus. The values of inductor and capacitor are inserted to the matrix correspondingly. 


\section{Chapter 4}

\section{Parallel Domain Decomposition Method}

In Chapter 2, several conventional simulation methods in power systems were reviewed and their limitations were also discussed. To address these issues, a simulation technique called Domain Decomposition is introduced and implemented in this chapter, closely following the references [55 57]. The presented method is able to obtain accurate simulation results through modelling the system in detail. Meanwhile, the computation time is hugely less than that of traditional EMT based simulators. Hence, the proposed method inherits the merits of both TS simulators and EMT simulators and is easier to apply.

The rest of this chapter is organized as follows: Section 4.1 introduces the domain decomposition. Then, the general solution of non-overlapping domain decomposition

method is described in Section 4.2. Finally, Section 4.3 presents the solution of special techniques applying with the application of the parallel domain decomposition method in power system simulation, i.e. system partition, latency, and initialization. 


\subsection{Domain Decomposition}

To effectively take advantages of modern parallel computing environments, algorithms must involve a large number of tasks which can be executed concurrently. Domain decomposition preconditioning techniques represent a very effective way of developing such algorithms. The domain decomposition method was first published in 1890 [58]. Domain decomposition methods are usually used to separate a large domain or a large system(Figure. 4.1) into several sub-domains. Therefore, a smaller problem on each sub-domain can be solved concurrently and individually [55 57]. The problems on the sub-domains are independent, which make domain decomposition methods suitable for the parallel computing.
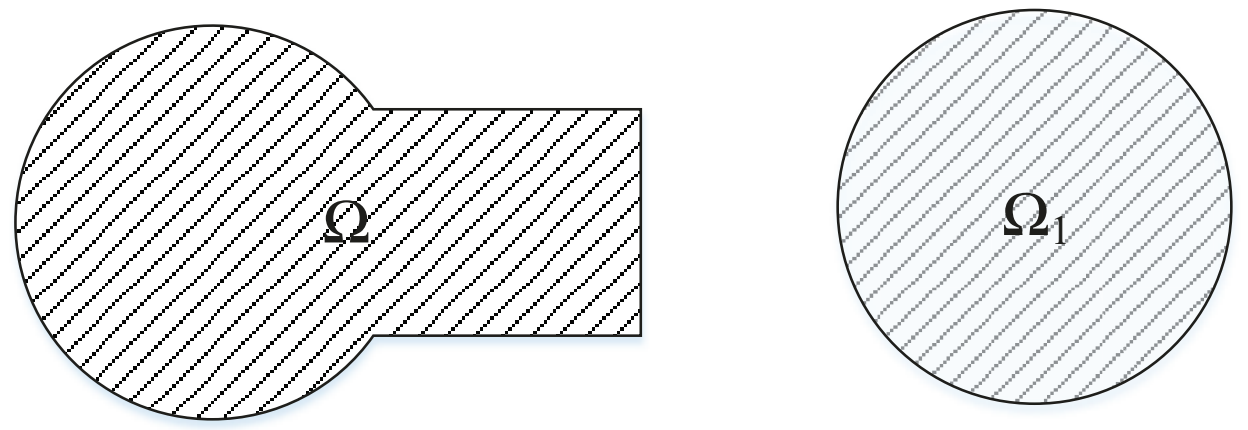

Figure 4.1: Block diagram of original whole system

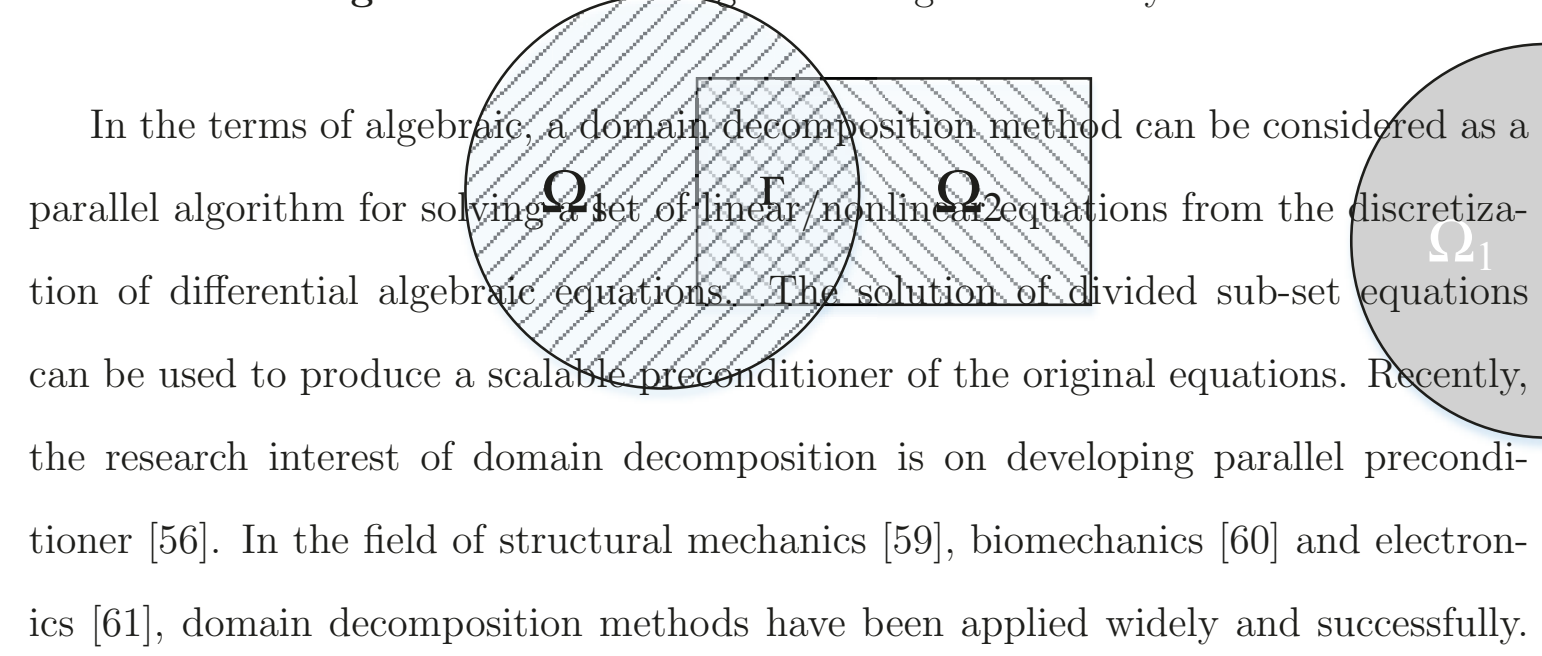


The parallelizable tasks are associated with subdomain solves. The traditional domain decomposition method is used to solve the time-harmonic Maxwell equations. The electromagnetic problem and the simulation of power system are similar. Both of these two problems are described by a differential equation set. Both of them can be divided into several parts easily. Due to the characteristic of power system, it is quite suitable for applying domain decomposition. There are still some differences between electromagnetic problem and simulation of power system. The power system contains not only differential equations but also algebraic equations. In addition, power system contains more types of variables besides electrical variables.

There are two basic approaches to develop domain decomposition preconditioners. The first one is the so-called non-overlapping approach (Figure. 4.2) and is characterized by the need to solve subproblems on disjoint subdomains. The second approach for developing domain decomposition preconditioners involves the solution of subproblems on overlapping subdomains(Figure. 4.3).
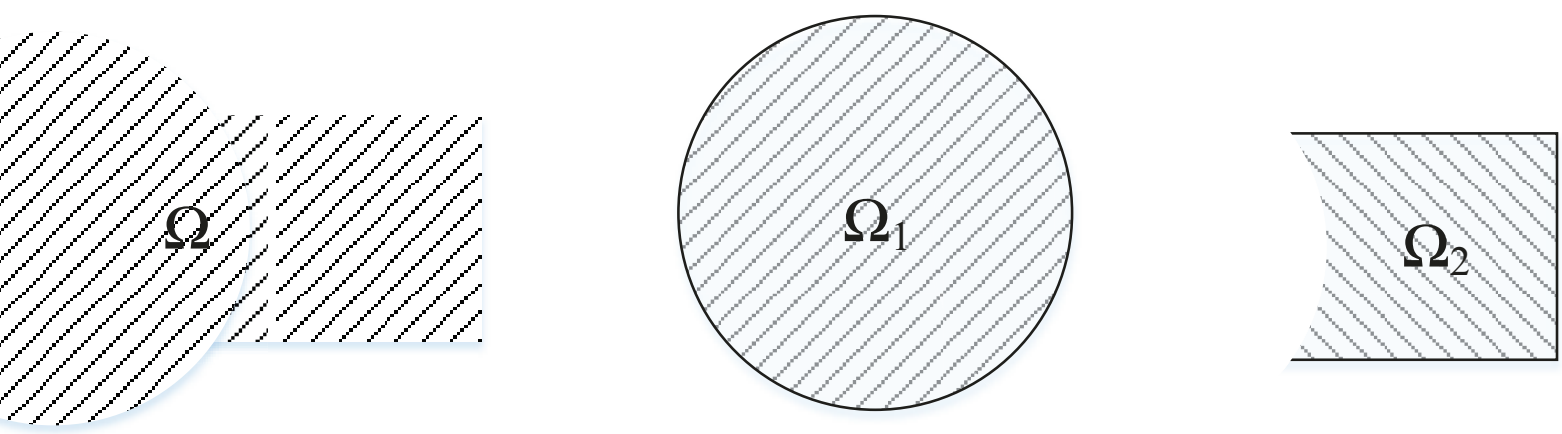

Figure 4.2: Non over lapping partition

Mhe overlapping mothod splits the computationaldemain into a number of overQ 2 apping subdomains, as shown in Figure

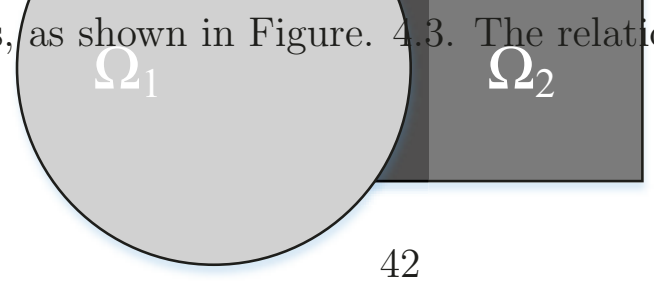
onship of each subdomain is 
In general, non-overlapping methods can be called as Schur complement methods and overlapping methods can be called as Schwarz methods. In Schur com-

plement methods, the equation set is partitioned into a number of non overlapping sub-domains. The variables in each sub-domains are split into interface and interior variables. The solution of the Schur complement gives the interface variables firstly. After that, the interior variables can be obtained by solving sub-domain equations concurrently. In Schwarz methods (overlapping method), the equation set is partitioned into a number of overlapping sub-domains and each sub-domain equations are solved alternately with an approximate solution. The solution is updated by propagating the solution of the sub-domains. Schwarz methods can be considered as the combination of Jacobi and Gasss-Seidel iterative methods [55, 56].

For such overlapping methods, it is always possible to replace the subproblem solution with a preconditioning evaluation. However, in parallel implementations, the amount of inter-processor communication is proportional to the amount of overlap. These methods lose some efficiency as the overlap becomes smaller. Theoretically, they are much worse in the case when there are jumps in coefficients. In this thesis, the non-overlapping method is employed due to the its advantages and the disadvantages of the overlapping method.

\subsection{Non-overlapping Domain Decomposition Method}

In numerical analysis, the Schur complement method, named after Issai Schur, is the basic and the earliest version of non-overlapping domain decomposition method, also called iterative substructuring. A finite element problem is split into non-overlapping subdomains, and the unknowns in the interiors of the subdomains are eliminated. The 
remaining Schur complement system on the unknowns associated with subdomain interfaces is solved by the conjugate gradient method.

There are two benefits of the Schur complement method. Firstly, the elimination of the interior unknowns on the subdomains, which is the solution of the Dirichlet problems, can be done in parallel. Secondly, passing to the Schur complement reduces condition number and thus tends to decrease the number of iterations. For secondorder problems, such as the Laplace equation or linear elasticity, the matrix of the subsystem has condition number of the order $1 / h^{2}$, where $h$ is the characteristic element size. The Schur complement, however, has condition number only of the order $1 / h$. For performances, the Schur complement method is combined with preconditioning, at least a diagonal preconditioner. The Neumann-Neumann method and the Neumann-Dirichlet method are the Schur complement method with particular kinds of preconditioner.

The Schur complement method for the non-overlapping domain decomposition is described as follows. The equation for any sub-system $\Omega_{i}$ can be written equivalent as:

$$
\left[\begin{array}{cc}
\boldsymbol{A}_{i}^{I I} & \boldsymbol{A}_{i}^{I \Gamma} \\
\boldsymbol{A}_{i}^{\Gamma I} & \boldsymbol{A}_{i}^{\Gamma \Gamma}
\end{array}\right]\left[\begin{array}{c}
\boldsymbol{x}_{i}^{I} \\
\boldsymbol{x}_{i}^{\Gamma}
\end{array}\right]=\left[\begin{array}{c}
\phi_{i}^{I} \\
\boldsymbol{\phi}_{i}^{\Gamma}
\end{array}\right]
$$

The matrix $\boldsymbol{A}_{i}^{I I}$ relates to the matrix of interior nodes and variables and $\boldsymbol{A}_{i}^{I \Gamma}, \boldsymbol{A}_{i}^{\Gamma I}$ matrix describe the coupling between interior and interface variables. The remaining $\boldsymbol{A}_{i}^{\Gamma \Gamma}$ represents the matrix of interface bus. $\boldsymbol{x}_{i}^{I}$ is the interior solution vector which represents the node voltage or variables inside sub-domain $\Omega_{s}$ and $\boldsymbol{x}_{i}^{\Gamma}$ is the interface variables which represent the node on the boundary of two adjacent sub-domain. 
The internal sub-system variables can be expressed as

$$
\boldsymbol{x}_{i}^{I}=\left(\boldsymbol{A}_{i}^{I I}\right)^{-1}\left(\phi_{i}^{I}-\boldsymbol{A}_{i}^{I \Gamma} \boldsymbol{x}_{i}^{\Gamma}\right)
$$

Similarly, all the sub-domains can be arranged in this way as interface variables in the bottom and interior variables are put in the diagonal one by one.

The equation for the whole system has been presented in Chapter. 3. The equation to be solved is denoted as:

$$
A x=b
$$

In the proposed method, the original large system is partitioned into a family of $P$ subsets plus one inter face, labeled $\Gamma$. Each subsystem is stored in each processor. If a system is correctly partitioned, for any pairs of subsystem $A_{1}, A_{2}$, no inner unknown of $A_{1}$ is directly coupled with any unknown of $A_{2}$. According to the decomposition, the unknown vector $x$ is reordered as:

$$
\boldsymbol{x}=\left[\boldsymbol{x}_{0}, \boldsymbol{x}_{1}, \ldots, \boldsymbol{x}_{P-1}, \boldsymbol{x}_{\Gamma}\right]^{t}
$$

Inside the subsystem, the order of unknowns will not be changed. Let $N_{i}$ be the number of inner unknowns of subsystem $i$ and $N_{s}$ the number of unknowns in interface. According to new ordination, Eq. (3.4) can be re-written in the terms of block 
matrices as follows:

$$
\left[\begin{array}{ccccc}
\boldsymbol{A}_{1}\left(a_{1} \ldots a_{n}\right) & 0 & \ldots & 0 & \boldsymbol{C}_{1}^{I \Gamma} \\
0 & \boldsymbol{A}_{2}\left(b_{1} \ldots b_{n}\right) & & 0 & \boldsymbol{C}_{2}^{I \Gamma} \\
\vdots & 0 & \ddots & 0 & \vdots \\
0 & 0 & \ldots & \boldsymbol{A}_{i}\left(y_{1} \ldots y_{n}\right) & \boldsymbol{C}_{i}^{I \Gamma} \\
\boldsymbol{D}_{1}^{\Gamma I} & \boldsymbol{D}_{2}^{\Gamma I} & \ldots & \boldsymbol{D}_{i}^{\Gamma I} & \boldsymbol{R}_{\Gamma \Gamma}\left(z_{1} \ldots z_{n}\right)
\end{array}\right]\left[\begin{array}{c}
\boldsymbol{x}_{1} \\
\boldsymbol{x}_{2} \\
\vdots \\
\boldsymbol{x}_{i} \\
\boldsymbol{x}_{\Gamma}
\end{array}\right]=\left[\begin{array}{c}
\boldsymbol{\phi}_{1} \\
\boldsymbol{\phi}_{2} \\
\vdots \\
\boldsymbol{\phi}_{i} \\
\boldsymbol{\phi}_{\Gamma}
\end{array}\right]
$$

The sizes and structure of each submatrics in (4.7) are:

1. $A_{1} \ldots A_{i} \in \mathbb{R}^{N_{i} \times N_{i}}$ are sparse matrices, which express the coupling of the inner unknown variables in subsystem $i$;

2. $C_{1} \ldots C_{i} \in \mathbb{R}^{N_{i} \times N_{s}}$ are sparse matrices, which express the coupling of the inner unknown variables of subsystem $i$ with the interface unknowns.

3. $D_{1} \ldots D_{i} \in \mathbb{R}^{N_{s} \times N_{i}}$ are sparse matrices, which express the coupling of the interface unknown variables with the inner unknowns of subsystem $i$.

4. $R \in \mathbb{R}^{N_{s} \times N_{s}}$ is a matrix describing coupling between interface variables;

5. $\boldsymbol{x} \in \mathbb{R}^{N_{i} \times N_{s}}$ is a vector describing the change of variables in each iteration.

Figure. 4.4 shows a simple partition example. The interface are 5 bus and 8 bus, which will be ordered into the end. This example is a domain with 4 buses in first subdomain and 3 buses in the second subdomain, to be solved with 2 processors.

Figure. 4.5 and Figure. 4.6 show the impact of new ordination. Figure. 4.5 illustrates the Jacobin matrix sparsity pattern before applying domain decomposition. 


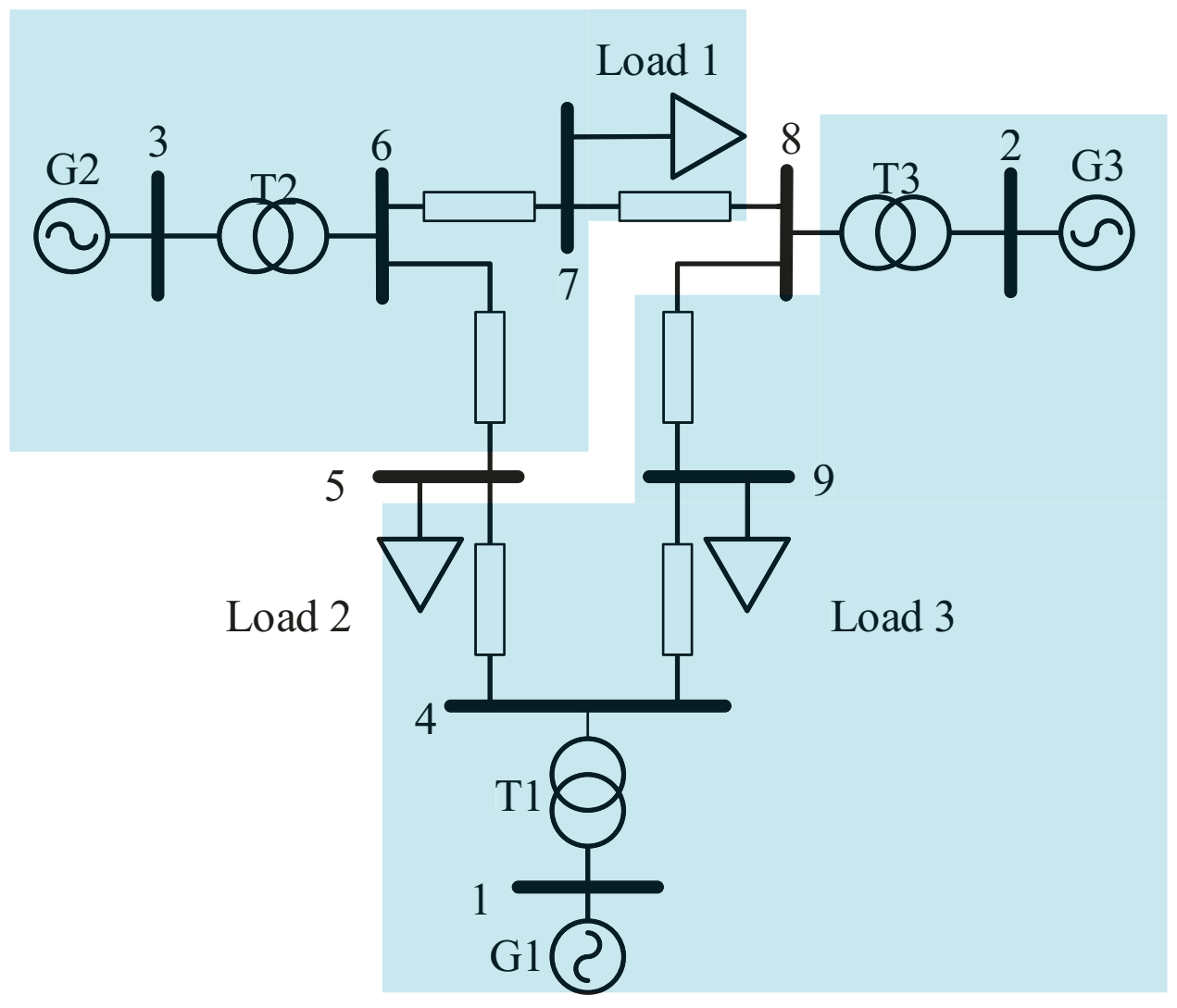

Figure 4.4: Example of system decomposition

\begin{abstract}
Although the majority data locates in diagonal, some outside data will affect calculation critically. The calculation speed cannot be improved by partitioning the matrix into small pieces. Figure. 4.6 represents the sparsity pattern after domain decomposition, $\boldsymbol{A}_{i}, \boldsymbol{C}_{i}, \boldsymbol{D}_{i}$ matrices are also circled to be represented. $\boldsymbol{C}$ and $\boldsymbol{D}$ matrix all locate on the margin of Jacobian matrix. All the $\boldsymbol{A}$ matrices locate on the diagonal, that is the same form of 4.7 .
\end{abstract}

\title{
4.2.1 Schur Complement Method
}

The Schur complement method is a direct parallel technique to eliminate the interior variables in each sub-domains. It develops a linear equation which only contains 


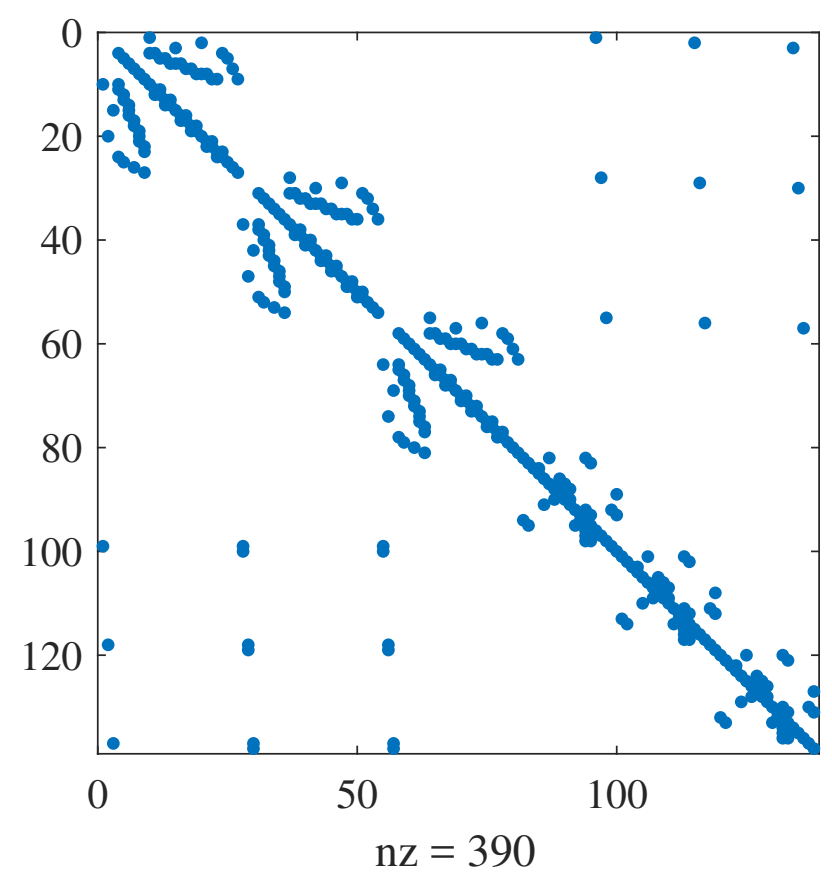

Figure 4.5: Sparsity pattern of original system

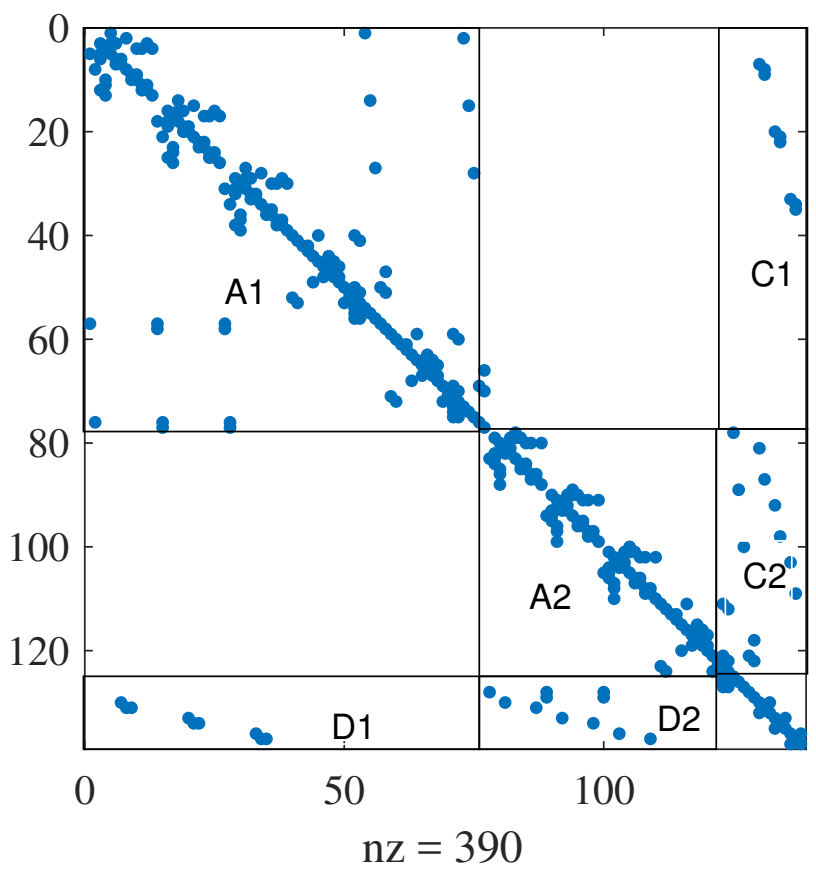

Figure 4.6: Sparsity pattern of partitioned system 
interface variables first based on Gaussian elimination method. The size of this linear equation is smaller comparing with the original one. Once the interface variables are obtained, the remaining sub-domains are decoupled, then the interior variables can be computed independently. The majority time usually takes on the solving the interface variables.

At first, the interface variable needs calculating. The last block equation of (4.7) can be written in scalar:

$$
\left[\boldsymbol{D}_{1}^{\Gamma I}, \boldsymbol{D}_{2}^{\Gamma I}, \cdots, \boldsymbol{D}_{i}^{\Gamma I}, \boldsymbol{R}^{\Gamma \Gamma}\right] \cdot\left[\boldsymbol{x}_{1}, \boldsymbol{x}_{2}, \cdots, \boldsymbol{x}_{i}, \boldsymbol{x}_{\Gamma}\right]^{t}=\phi_{\Gamma}
$$

Eq. (4.8) can be transferred by Gaussian elimination to:

$$
\left[0,0, \cdots, 0, \tilde{\boldsymbol{R}}_{\Gamma \Gamma}\right] \cdot\left[\boldsymbol{x}_{1}, \boldsymbol{x}_{2}, \cdots, \boldsymbol{x}_{i}, \boldsymbol{x}_{\Gamma}\right]^{t}=\tilde{\boldsymbol{\phi}}_{\Gamma}
$$

which equals,

$$
\tilde{\boldsymbol{R}}_{\Gamma \Gamma} \cdot \boldsymbol{x}_{\Gamma}=\tilde{\phi}_{\Gamma}
$$

where

$$
\tilde{\boldsymbol{R}}_{\Gamma \Gamma}=\boldsymbol{R}_{\Gamma \Gamma}-\sum_{i=0}^{P-1} \boldsymbol{D}_{i}^{\Gamma I} \boldsymbol{A}_{i}^{-1} \boldsymbol{C}_{i}^{I \Gamma}
$$

and

$$
\tilde{\boldsymbol{\phi}}_{\Gamma}=\boldsymbol{\phi}_{\Gamma}-\sum_{i=0}^{P-1} \boldsymbol{D}_{i}^{\Gamma I} \boldsymbol{A}_{i}^{-1} \boldsymbol{\phi}_{i}
$$

The Eq. (4.9) can be used to obtain the interface variables $x_{\Gamma}$ before the interior unknowns. Once $x_{\Gamma}$ is known, each unknown variables for $i$ subsystem $\left(x_{i}\right)$ can be determined by:

$$
\boldsymbol{A}_{i} \boldsymbol{x}_{i}=\phi_{i}-C_{i}^{I \Gamma} \boldsymbol{x}_{\Gamma}
$$


The evaluation of $\tilde{\boldsymbol{R}}_{\Gamma \Gamma}$ and $\tilde{\boldsymbol{\phi}}_{\Gamma}$ can affect the efficiency of proposed algorithm directly. Its explicit computation is not possible except for a small system. For each term,

$$
\boldsymbol{a}_{i}=\boldsymbol{D}_{i}^{\Gamma I} \boldsymbol{A}_{i}^{-1} \boldsymbol{C}_{i}^{I \Gamma}
$$

it can be evaluated column by column, without explicitly calculation of $\boldsymbol{A}_{i}^{-1}$. From the first column to $N_{s}$, solve for auxiliary vector $t$ in equation:

$$
\boldsymbol{A}_{i} \boldsymbol{t}=\left[\boldsymbol{C}_{i}^{I \Gamma}\right]_{c}
$$

where $[\cdot]_{c}$ is column $\mathrm{c}$ of a matrix. Then:

$$
\left[\boldsymbol{a}_{\boldsymbol{i}}\right]_{c}=\boldsymbol{D}_{i}^{\Gamma I} \boldsymbol{t}
$$

As some column of the $C^{I \Gamma}$ matrix are null, some effort can be saved. Once the contribution of each processor is ready, they are subtracted from $\boldsymbol{A}_{\Gamma}$. Similarly for $\tilde{\phi}_{\Gamma}$,

$$
\boldsymbol{b}_{i}=\boldsymbol{D}_{i}^{\Gamma I} \boldsymbol{A}_{i}^{-1} \boldsymbol{\phi}_{i}
$$

$\boldsymbol{b}_{i}$ is calculated in each processor as:

$$
\begin{gathered}
\boldsymbol{A}_{i} \boldsymbol{t}=\boldsymbol{\phi}_{i} \\
\boldsymbol{b}_{i} \boldsymbol{t}=\boldsymbol{D}_{i}^{\Gamma I} \boldsymbol{t}
\end{gathered}
$$


Take two sub-systems as an example, the linear system can be written as:

$$
\left[\begin{array}{ccc}
\boldsymbol{A}_{11} & 0 & \boldsymbol{C}_{1}^{I \Gamma} \\
0 & \boldsymbol{A}_{22} & \boldsymbol{C}_{2}^{I \Gamma} \\
\boldsymbol{D}_{1}^{\Gamma I} & \boldsymbol{D}_{2}^{\Gamma I} & \boldsymbol{R}_{\Gamma \Gamma}
\end{array}\right]\left[\begin{array}{l}
\boldsymbol{x}_{1} \\
\boldsymbol{x}_{2} \\
\boldsymbol{x}_{\Gamma}
\end{array}\right]=\left[\begin{array}{l}
\boldsymbol{\phi}_{1} \\
\boldsymbol{\phi}_{2} \\
\boldsymbol{\phi}_{\Gamma}
\end{array}\right]
$$

Thus, the Schur complement method can be represented using (4.10) as follows:

$$
\tilde{\boldsymbol{R}}_{\Gamma \Gamma} \boldsymbol{X}_{\Gamma}=\boldsymbol{\phi}_{\Gamma}-\boldsymbol{A}_{\Gamma 1} \boldsymbol{A}_{11}^{-1} \phi_{1}-\boldsymbol{A}_{\Gamma 2} \boldsymbol{A}_{22}^{-1} \boldsymbol{\phi}_{2}
$$

where $\tilde{\boldsymbol{R}}$ is the Schur complement matrix:

$$
\tilde{\boldsymbol{R}}_{\Gamma \Gamma}=\boldsymbol{R}_{\Gamma \Gamma}-\boldsymbol{D}_{1}^{\Gamma I} \boldsymbol{A}_{11}^{-1} \boldsymbol{C}_{1}^{I \Gamma}-\boldsymbol{D}_{2}^{\Gamma I} \boldsymbol{A}_{22}^{-1} \boldsymbol{C}_{2}^{I \Gamma}
$$

Once the values on the interface $\left(\boldsymbol{x}_{\Gamma}\right)$ are known, the following two relations can be used to calculate interior values in parallel by using (4.13).

$$
\begin{aligned}
& \boldsymbol{A}_{11} \boldsymbol{x}_{1}=\phi_{1}-\boldsymbol{C}_{1}^{I \Gamma} \boldsymbol{x}_{\Gamma} \\
& \boldsymbol{A}_{22} \boldsymbol{x}_{2}=\phi_{2}-\boldsymbol{C}_{2}^{I \Gamma} \boldsymbol{x}_{\Gamma}
\end{aligned}
$$

Then $\boldsymbol{x}_{1}$ and $\boldsymbol{x}_{2}$ can be obtained:

$$
\begin{aligned}
& \boldsymbol{x}_{1}=-\boldsymbol{A}_{11}^{-1} \boldsymbol{C}_{1}^{I \Gamma} \boldsymbol{x}_{\Gamma}+\boldsymbol{A}_{11}^{-1} \boldsymbol{\phi}_{1} \\
& \boldsymbol{x}_{2}=-\boldsymbol{A}_{22}^{-1} \boldsymbol{C}_{2}^{I \Gamma} \boldsymbol{x}_{\Gamma}+\boldsymbol{A}_{22}^{-1} \boldsymbol{\phi}_{2}
\end{aligned}
$$


The inverse calculation of matrices in this chapter is realized by LU decomposition method. This is a function which can decompose a square matrix into a product of lower triangular matrix and an upper triangular matrix. With the help of this decomposition, a linear equation can be solved in a more efficient way. LU decomposition of $\boldsymbol{A}_{11}$ and $\boldsymbol{A}_{22}$ are finished in parallel, then the total calculation time must be much less than original matrix $\boldsymbol{A}$, which is the basis to implement parallel simulations for power systems.

\subsection{Parallel Implementation}

To exploit the characteristics of DD, a parallelization technique is applied. Based on the discussion of Section. 4.2, there are two opportunities to realize the parallelization in the DD algorithm, including the LU decomposition of corresponding $A_{i}$ matrix and the calculation of interior variables in each subsystems. Therefore, the implementation of this proposed algorithm is shown in Figure. 4.7. In the flow chart, 'initialization variables' block includes the modelling of the power system, initial condition calculation and matrices preprocessing. 'Update Jacobian matrix' block corresponds to updating each $A_{i}, C_{i}$ and $D_{i}$ matrices and calculate the LU decomposition of corresponding $A_{i}$ on each processor. 'Calculate Schur complement' block means the realization of (4.11). 'Calculate interior variables' block represents the calculation of 4.13).

In theory, the simulation time should be reduced linearly with the increase of the number of cores. However, in reality, with the increase of number of cores, communication overhead becomes larger. The performance of parallelization depends on the communication overhead between each processor, the level of parallelization, processors number, etc. In this thesis, the modelling time and re-ordering time are not 


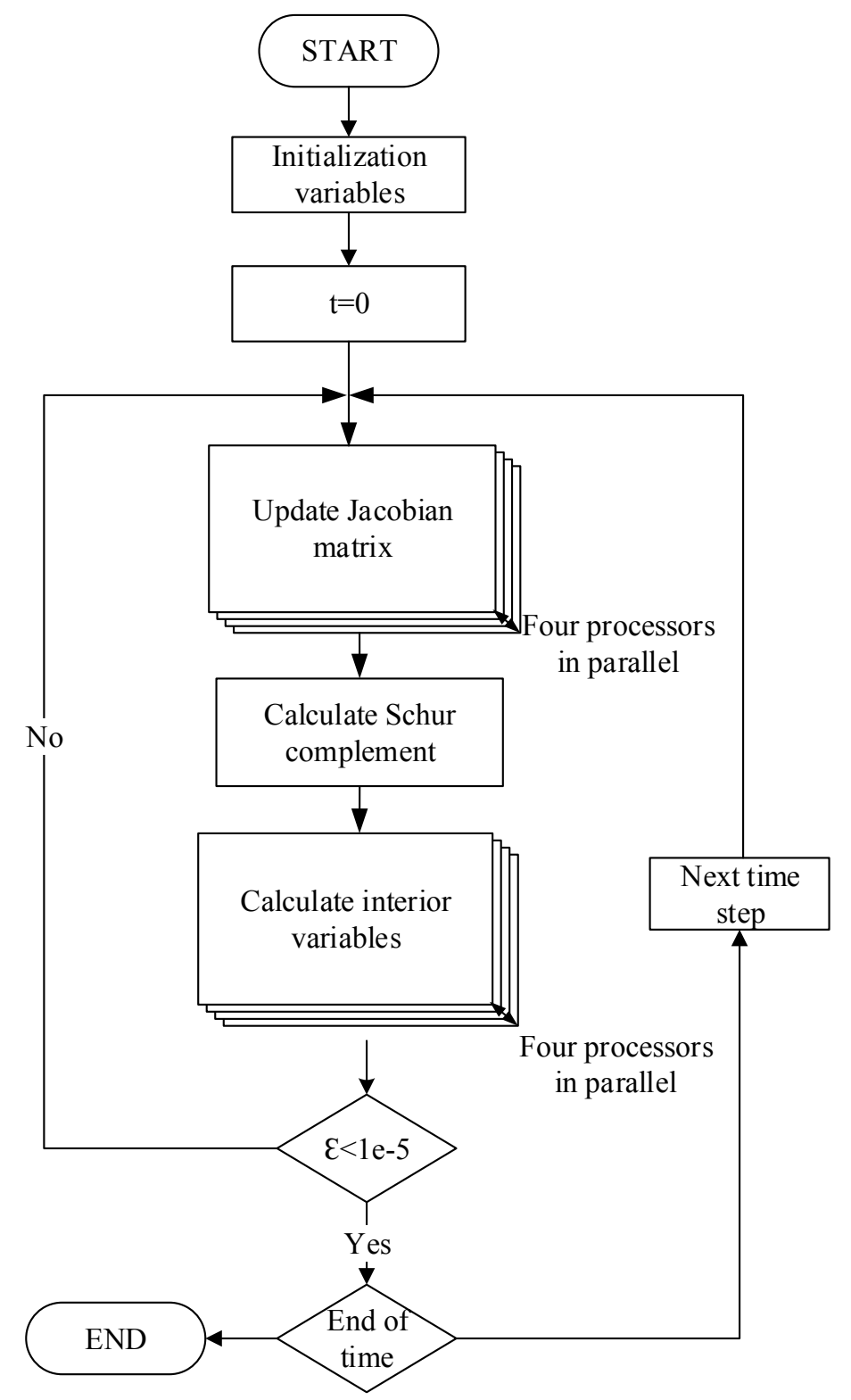

Figure 4.7: The Parallel Domain Decomposition Algorithm

included in the CPU time, due to small computation time comparing with iteration time. Parallel calculation was finished with the help of Parallel Computing Toolbox in MATLAB. There are two most useful parallel programming functions in Matlab. They are parfor (parallel for) and spmd (single program multiple data). There is a limit in parfor, that each iteration must be independent with other iterations. This 
limitation is quite strict for this thesis. However, spmd allows serial programming and parallel programming seamlessly, which makes it easier to implement parallel programming [63]. The execution of spmd can be divided into three periods: setup, execution and collection as Figure. 4.8.

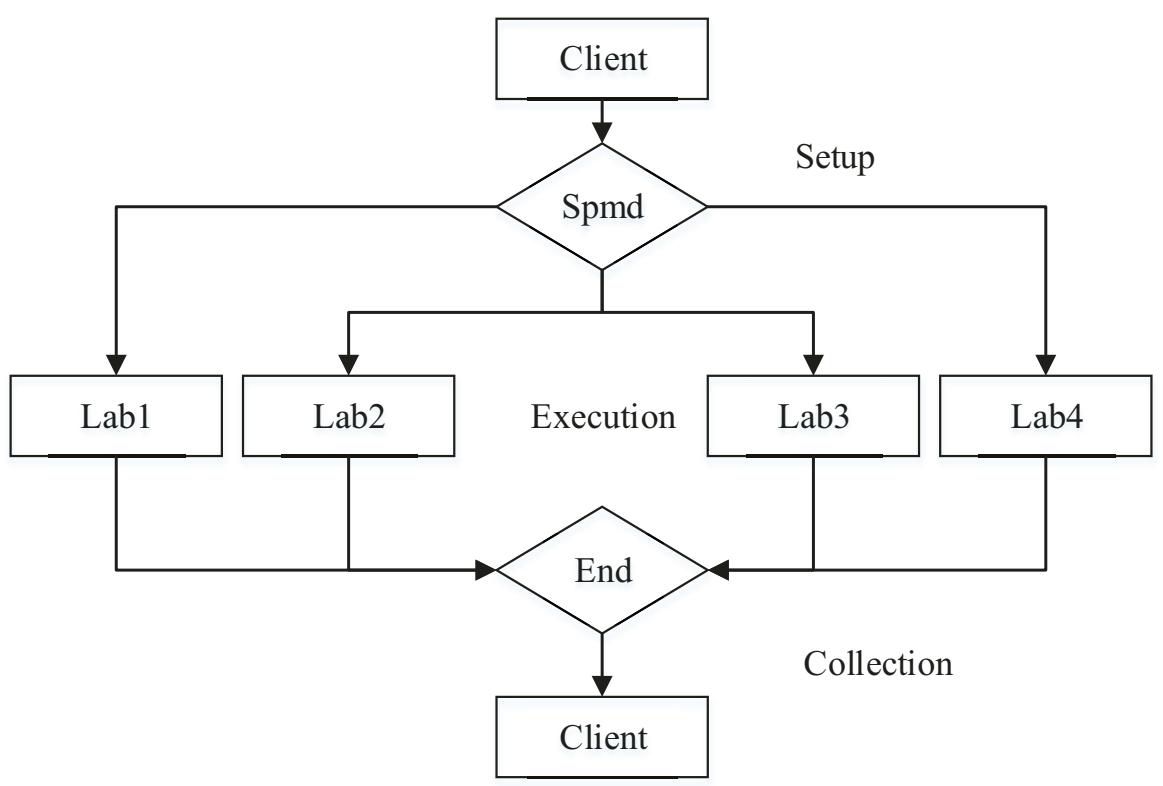

Figure 4.8: Spmd operation mechanism with four processors

We use $T_{\text {start }}$ to represent setup time. $T_{\text {run }}$ means the time spending on running the same code in different processors. $T_{\text {collect }}$ means the time to collect all the results. $T_{\text {start }}$ is always fixed, around $0.055 \mathrm{~s}$. $T_{\text {collect }}$ depends on the size of data. All the data inside spmd loop does not share with each other workers, but data can be sent and shared by calling Broadcast function 64. Therefore, if communication time is larger than individual calculation time, parallel calculation cannot help improving simulation speed. 


\subsection{Special Characteristics of Domain Decomposi- tion Applied to Power System Simulation}

To apply the domain decomposition based on Schur complement method to power system simulations, there are still several special cases to be exploited, such as system partitioning, latency and initialization. These cases are specifically related to the characteristic of power systems. This section will deal with these specific characteristic not only to apply the introduced domain decomposition method to power system simulation, but also to increase the efficiency of simulation.

\subsubsection{System Partitioning}

To obtain the best performance of domain decomposition, an algorithm is necessary to be used to partition the system into nearly balanced parts. When the size of subsystems is similar, all the processors run in the highest efficiency without waiting for data exchange. Consider a power system with $n$ buses that needs to be separated into $m$ sub-systems, where $m$ is the number of processors in CPU. K-means algorithm can be used to divided $n$ buses into $m$ clusters. K-means algorithm is a popular and simple clustering algorithm which can group buses by electrical distance. The electrical distance is defined in [65]. At the beginning, $m$ points are given manually for the centerpoint of each cluster. Each bus can only be included into one cluster. As Figure. 4.9, IEEE 39 bus system can be divided into three groups with same buses number. Each bus is assumed to be connected with similar transmission and loads.

The aforementioned algorithm aims to partition a power system based on physical

location. For a power system, it consists of electric components interfacing with each 


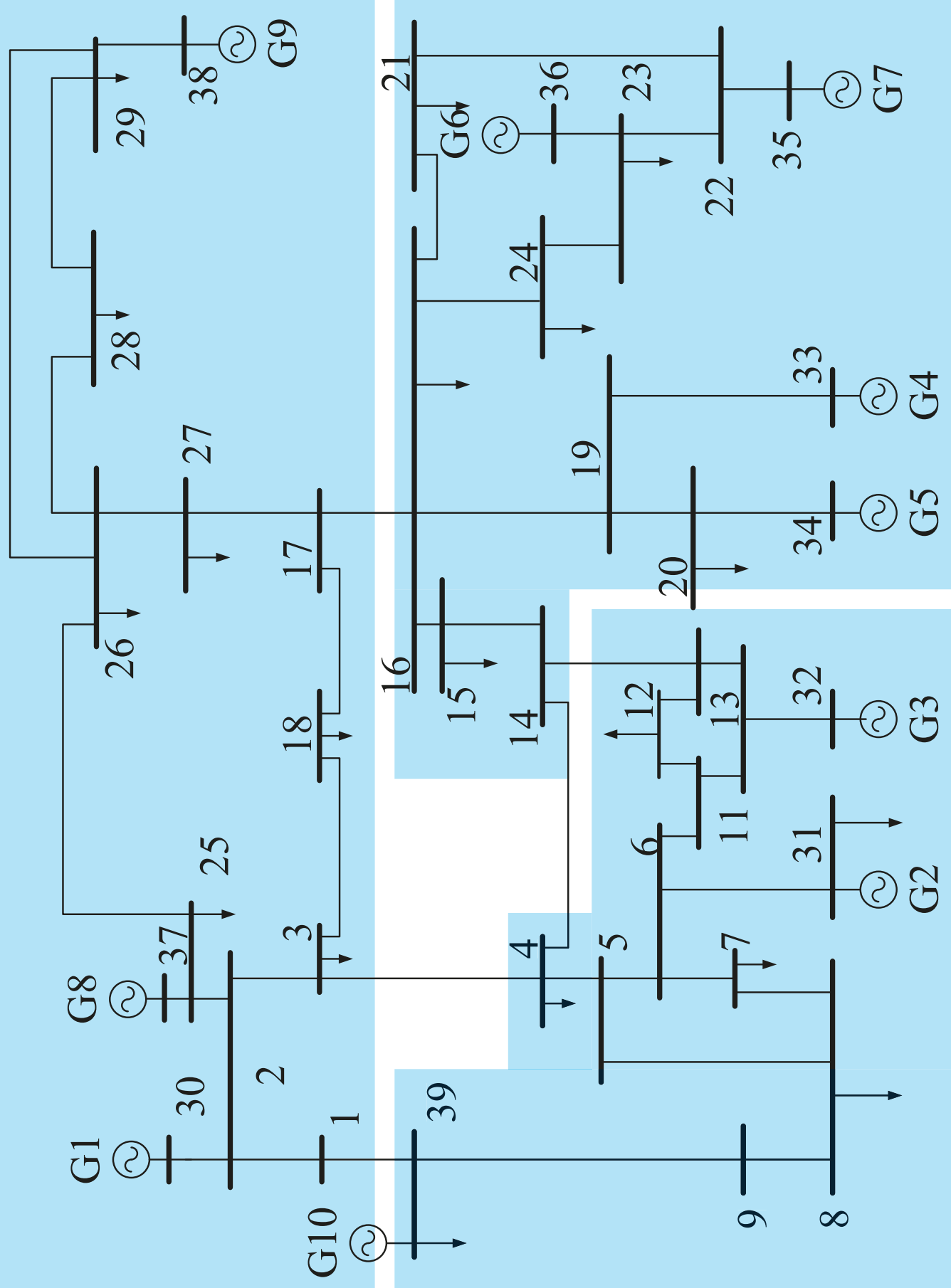

Figure 4.9: Example of partition of IEEE 39 bus system 


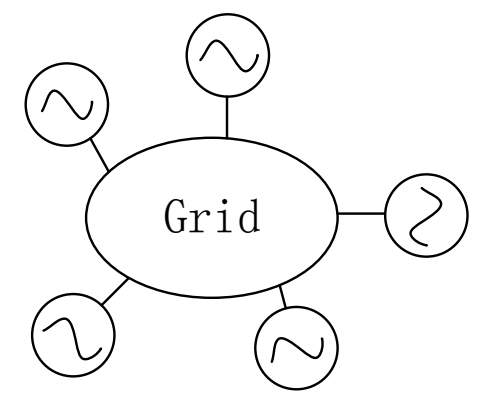

Figure 4.10: Decomposed power system

other through the network. The proposed algorithm applies a topological decomposition separating all the machines to one sub-domain. The partition algorithm is shown in Figure. 4.10, For each machine, the interface variable is the current through that bus and for the grid, the interface variables are the voltage of the bus which are connected by machines. The new partition layout is extremely helpful when applying DD method since it allows less computation. For the processor which stores the matrix for the grid, it does not change with time approaching or with Newton iteration. LU decomposition of $\boldsymbol{A}_{1}$ and some items in 4.11 can be reused during the whole simulation. For a matrix being large (since many buses, transmission lines exist in the grid), repeating the LU decomposition would impact the efficiency. In addition, this partitioning method does not require complicated algorithm. For the example used in Section. 4.2, the new proposed partitioning method is shown in Figure. 4.11. Machines G1,G2, and G3 are separated from the whole system.

\subsubsection{Latency}

Different devices in a power system have different time constants. Based on this characteristic, latency technology can be applied to domain decomposition. Decomposition becomes more attractive and efficient when blocks are latent. From the 


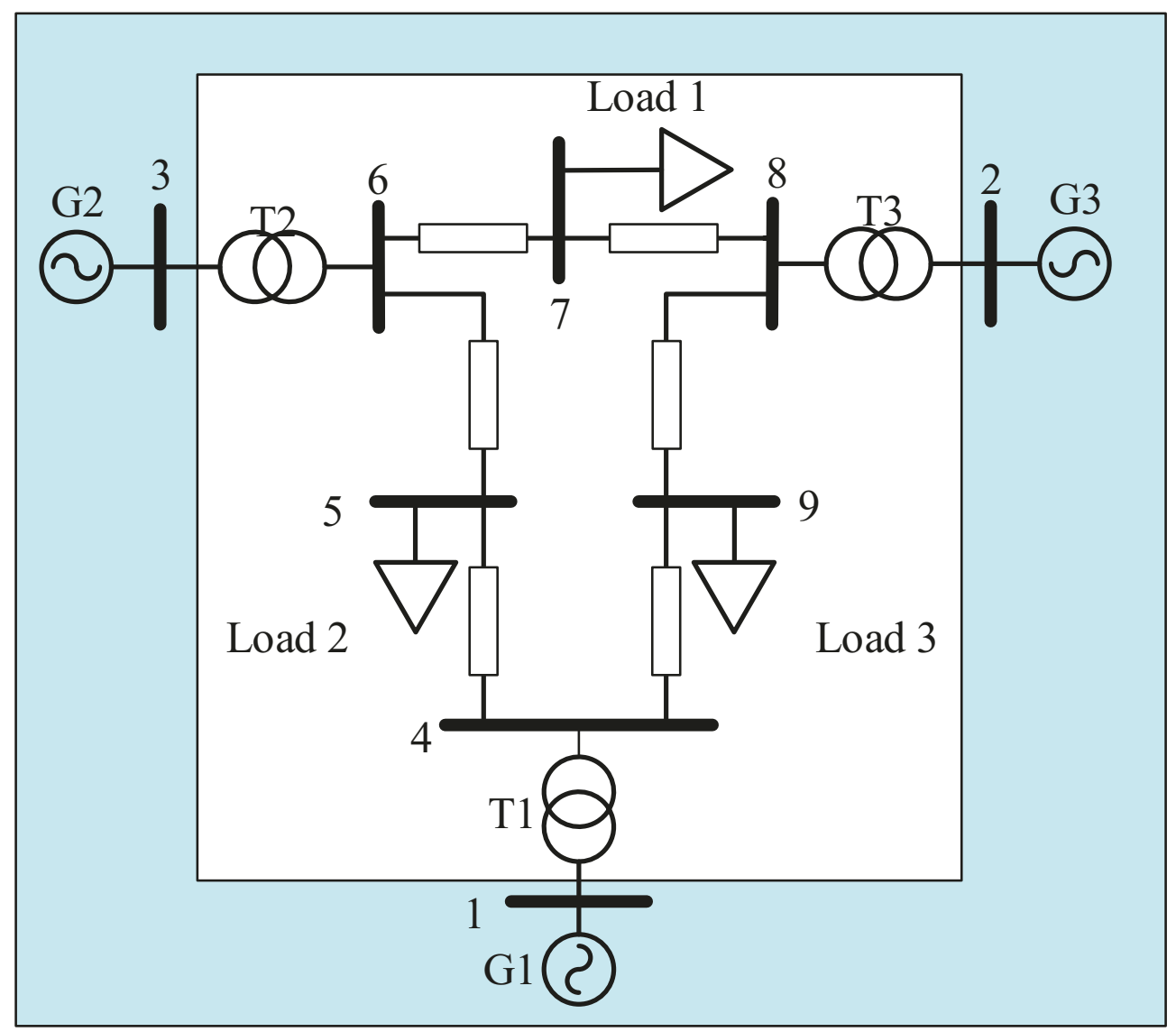

Figure 4.11: Example of new partitioning method

previous section, we solve a set of linear equations, arising from discretization and linearization of original equations, therefore three different categories of number in the Jacobian matrix: constant, elements changing only with each time-step approaching and elements changing with each NR iteration. In additional, some blocks can converge faster than others, then these blocks keep unchanged in NR iteration. If all the elements in block matrix are fixed during each NR iteration, this block is considered as latent. Due to the characteristic of latent, we only need to perform LU decomposition for latent block once and then combine the result with other parts. Identification of unchanged elements are quite simple in power system, due to the existence of governor, synchronous machines always have longer response time, such 
as several seconds, while power electronic components like converters change in the frequency of $3 \mathrm{kHz}$, whose time constant is quite small. Another method is testing the convergence speed of each block, then we can determine the block as being latent. It is not necessary to do LU decomposition for small time constant devices in every iteration and even every time-step. Therefore, more time is saved during each time-step.

Once the latency is introduced in domain decomposition, these subdomains have different timestep. Due to the asynchronous between subdomains, the poles of orig-

inal differential equations change. It changes the constantly stable system into the conditional stable model. Therefore, the stability of the model should be judged. Before applying latency, we must make sure the difference of time constant between two subdomains is large. An indicator can be used to monitor the change of voltage to make sure the boundary conditions of these two subdomains truly converge. Once the system comes into an instability area, the latency must be reduced. In addition, latency can only be applied in the steady state. When the fault happens, even a very small latency may lead to instability.

\subsubsection{Initialization}

To analyze the transient change correctly, we need to calculate the initial condition. In traditional, we make all the differential equations equal zero and then solve the algebraic equations. The rotating angle keeps changing, therefore the traditional method cannot be used. Power flow calculation can help us obtain the terminal voltage and delivered(consumed) power, which is discussed in Appendix. A. Based on the voltage and power, other state variables of machine can be calculate correspondingly.

This section will summarize the procedure to calculate these variables. In this section, all the values are in per unit. The terminal active power $P$, reactive power 
$Q$ and voltage $V$ are obtained from power flow calculation. Therefore, the terminal current $I$ :

$$
I=\frac{\sqrt{\left(P^{2}+Q^{2}\right)}}{V}
$$

And factor angle $\theta$ as follows

$$
\theta=\cos ^{-1}\left(\frac{P}{V I}\right)
$$

Next, the rotating angle $\delta$ can be computed from

$$
\delta=\tan ^{-1}\left(\frac{X_{q} I \cos \theta-R_{a} I \sin \theta}{V+R_{a} I \cos \theta+X_{q} I \sin \theta}\right)
$$

Once we know the rotating angle, the $d q$ components are obtained easily:

$$
\begin{aligned}
& V_{d}=E \sin \delta \\
& V_{q}=E \cos \delta \\
& i_{d}=I \sin (\delta+\theta) \\
& i_{q}=I \cos (\delta+\theta)
\end{aligned}
$$

The relationship between variables of synchronous machine is shown in Figure. 4.12 . 


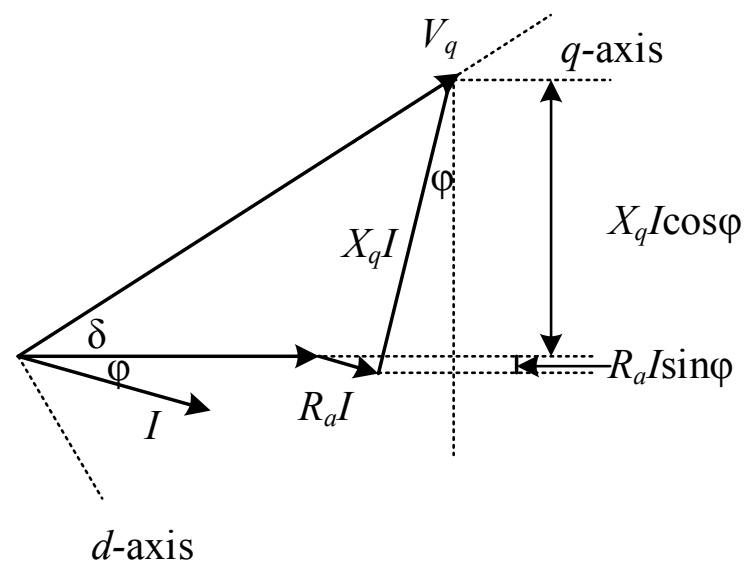

Figure 4.12: Phasor diagram of synchronous machine in steady state 


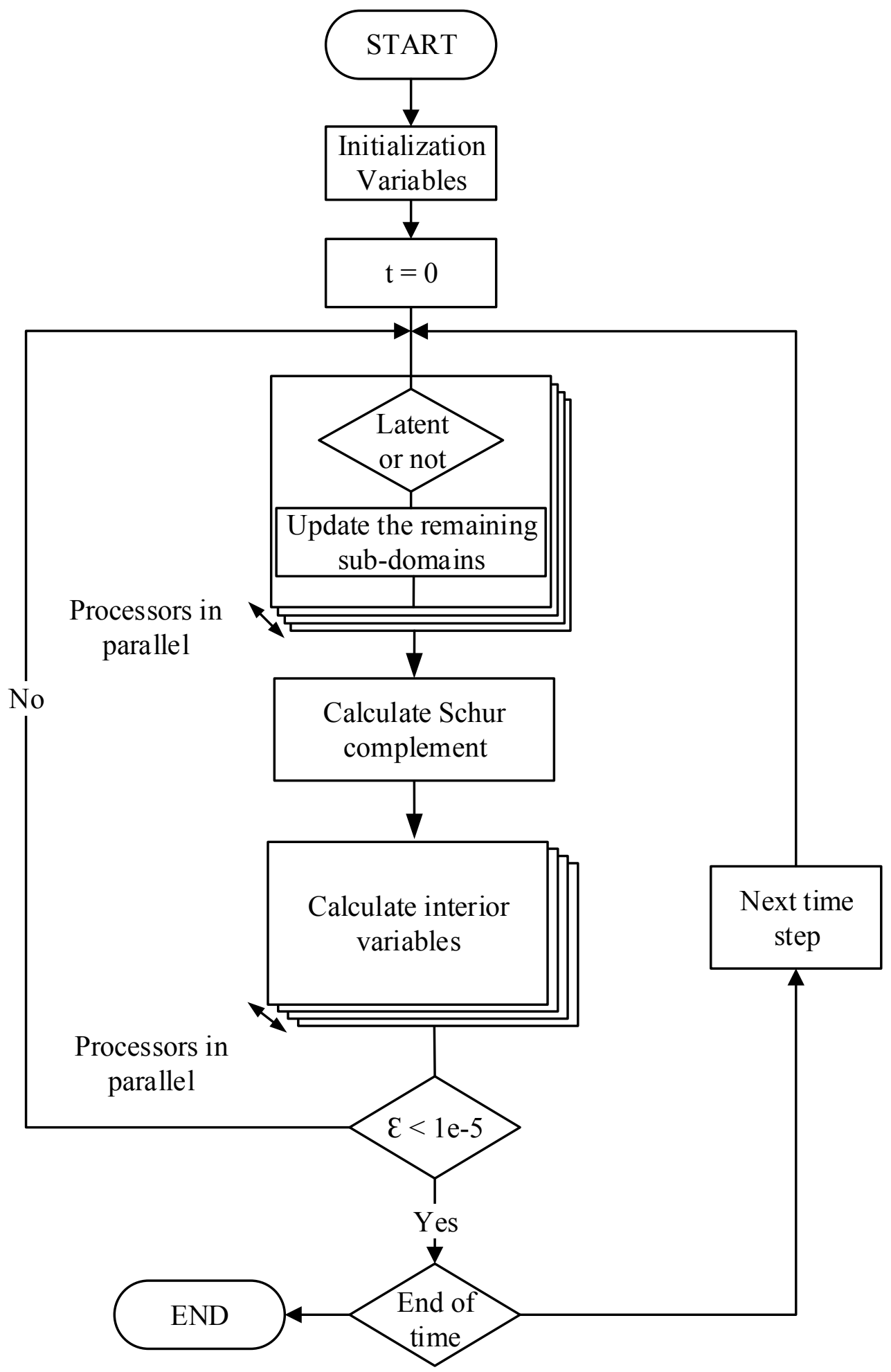

Figure 4.13: The Parallel Domain Decomposition Algorithm with latency 


\section{Chapter 5}

\section{Simulation Result Analysis and Comparison}

In this chapter, four numerical examples are presented to demonstrate the accuracy and efficiency of the proposed method. The simulations were done with a tolerance of 0.0001 for convergence in Newton iteration. The time step in all simulations is 10us. Typically, the practical size of power transmission system is below one thousand bus. In some commercial software, the limitation of number of bus usually ranges from 200 to 900 . The final test case is to validate the proposed algorithm in large system. This chapter presents the results of power system simulations using the following five algorithms for comparison:

- Traditional Electromagnetic Transient Simulation (EMT): This method is realized in Matlab/Simulink. All the components are modelled in the EMT type. The CPU time is for reference, cause the operating mechanisms are different from following algorithms. The simulation result is considered as the benchmark.

- Original Matrix (Org): the Jacobian matrix is updated in each iteration and time step. The equation set is solved simultaneously. 
- Normal Parallel Domain Decomposition (DD) algorithm shown in Figure. 4.7. the Jacobian matrix is updated in each iteration and each time step and the equation set is solved in parallel.

- Parallel Domain Decomposition with Latency (PDDL) algorithm shown in Figure. 4.13 the Jacobian matrix is updated when it is necessary and the equation set is solved in parallel.

- New Partition based Parallel Domain Decomposition (NDD): the Jacobian matrix is updated in each iteration and time step and the equation set is solved in parallel. The model of the grid is fixed and stored in the first processor.

All the numerical examples are executed in Matlab(R2016b) on the platform with Intel Core ${ }^{T M}$ i7-2600 $\mathrm{CPU}(3.4 \mathrm{GHz})$ and 16-GB RAM. The operation system is $64 \mathrm{bit}$ Windows 7 .

\subsection{Numerical Stability}

The trapezoidal integration method is chosen due to the reason that it has better stability and accuracy. Integration methods such as Backward Euler, Forward Euler, and R-K methods are called explicit methods. Explicit methods calculate the state of a system at a later time $(t+1)$ depending on the state of the system at the current time $(\mathrm{t})$. There is a significant limitation of the explicit integration methods that they are not A-stable. The time step is strictly restricted by the time constant of the system. In other words, the truncation error of these methods keeps increasing. The solution finally goes to infinite unless a very small time step is used.

The trapezoidal rule as the simplest implicit integration method, calculates the state at the current time based on the previous time step state as well as the current 
state (unknown). The trapezoidal method is A-stable. The time step only affects the accuracy but doest not affect the numerical stability. When a larger time step is applied, high frequency signals are filtered out. Other implicit integration methods with higher order have not been widely used in power systems due to the difficulty in programming and less numerical stability.

\subsection{Performance Index}

\subsubsection{Accuracy Index}

To demonstrate the accuracy of each algorithm, the results obtained by using different algorithms are compared with Simulink's results. The following normalized Euclidian norm is used to define the accuracy index:

$$
e=\frac{\|x-\hat{x}\|}{\operatorname{size}(x)}
$$

where $e$ is the norm of the error and $\operatorname{size}(x)$ is the dimension of vector $x . x$ and $\hat{x}$ are vectors of simulation result and benchmark, respectively. The smaller accuracy index, the better accuracy performance it is.

\subsubsection{Efficiency Index}

To demonstrate the efficiency of each algorithm, the efficiency index is computed by:

$$
\text { Speedup }=\frac{C P U \text { time }(1 \text { core })}{C P U \text { time }(M \text { cores })}
$$

Eq. (5.2) shows how faster is the parallel computation. 


\subsection{Case Study I: Small-Scale Power System}

The first test case is based on a small-sacle power system: IEEE 3-generator 9-Bus test system shown in Figure. B.1. This system contains nine buses, six transmission lines, three transformers, three constant impedance loads, and three synchronous generation machines with IEEE type-1 exciters. The total load demand is $315 \mathrm{MW}$ and 115 MVAR. The synchronous machines are modelled in details including voltage regulators and excitation systems. This system has 138 state variables in total, 57 states for generators and 81 states for the grid. After applying normal partitioning method, the first subsystem has 77 variables and the second subsystem has 52 variables. For the interface, there are 9 variables. This test case starts at all zero initial condition. There are two disturbances added to this small-scale power system to test the accuracy.

- Large disturbance: there is a three-phase short-circuit fault happening to the bus 4 . The fault started at $0.05 \mathrm{~s}$ and was cleared at $0.08 \mathrm{~s}$. The fault resistance is $0.0529 \Omega\left(10^{-4} \mathrm{pu}\right)$.

- Small disturbance: All machines start at zero rotating speed. The generators will produce $315 \mathrm{MW}$ active power and 115MVAR reactive power in total.

\subsubsection{Accuracy}

Figure. 5.1, Figure. 5.2, and Figure. 5.3 summarize the performance of transient changes of both the start of machine and disturbance at 0.05s. As expected, the simulation results are the same as simulink's results. The simulations were finished using the parameter in Table. B.1. Blue line is the result from Matlab/Simulink as the reference value. Red circle is the result from DD method. Yellow cross and purple star are the results from NDD and PDDL methods, respectively. As seen in these 
results, all methods are in good agreement. Figure. 5.1 and Figure. 5.2 show the acceleration of synchronous machine 1 . The output voltage of generator 1 starts rest state and increases rotating speed to the reference value.

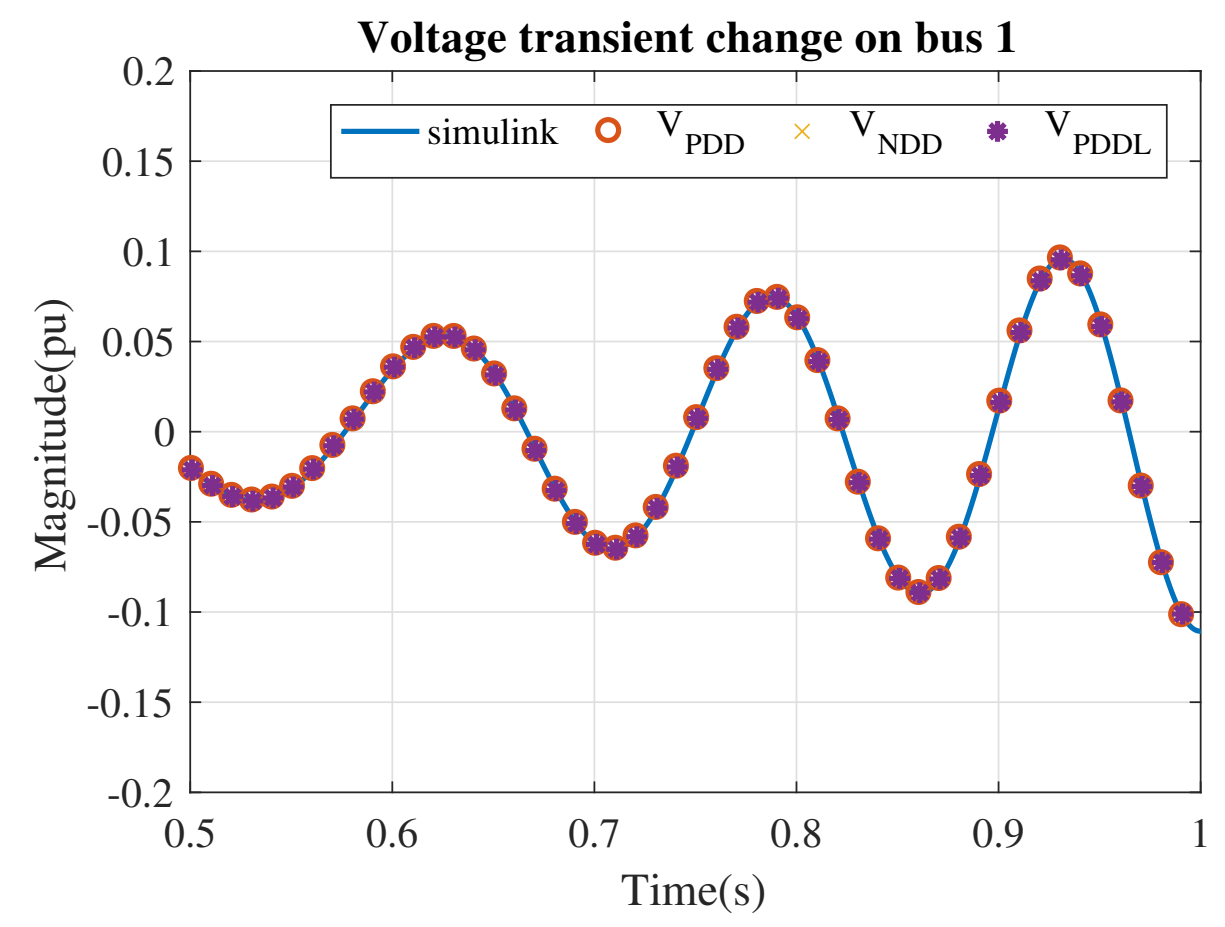

Figure 5.1: Voltage transient change at bus 1

Figure. 5.3 shows a transient change on the bus 1 when a fault happens at bus 4 . The voltage decreases to around $0.8 \mathrm{pu}$ and raises back to $1 \mathrm{pu}$ after fault clearance. Since the simulation results are almost the same, in the following figure, results of DD, NDD, and PDDL are combined into one group called 'M-file'.

For the DD and NDD method, the accuracy index are $4.15 \times 10^{-13}$ and $4.65 \times 10^{-13}$. The accuracy index should not change much due to the same calculation mechanism and modelling details. The only difference for these two methods is the order of variables. For the PDDL method, the accuracy index is $2.808 \times 10^{-6}$. Since the latency is applied to some components in the system, the Jacobian matrix cannot be 


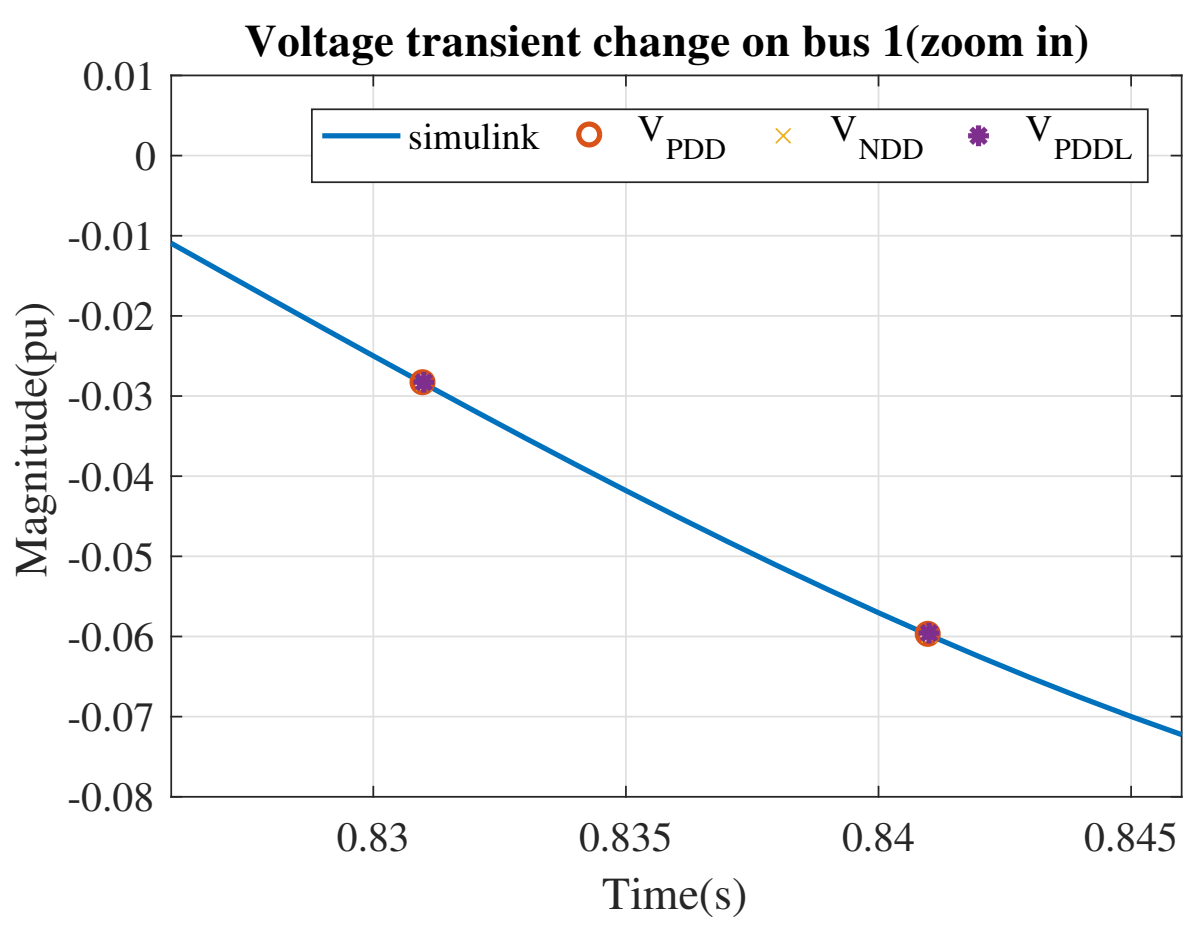

Figure 5.2: Voltage transient change at bus 1 (zoom in)

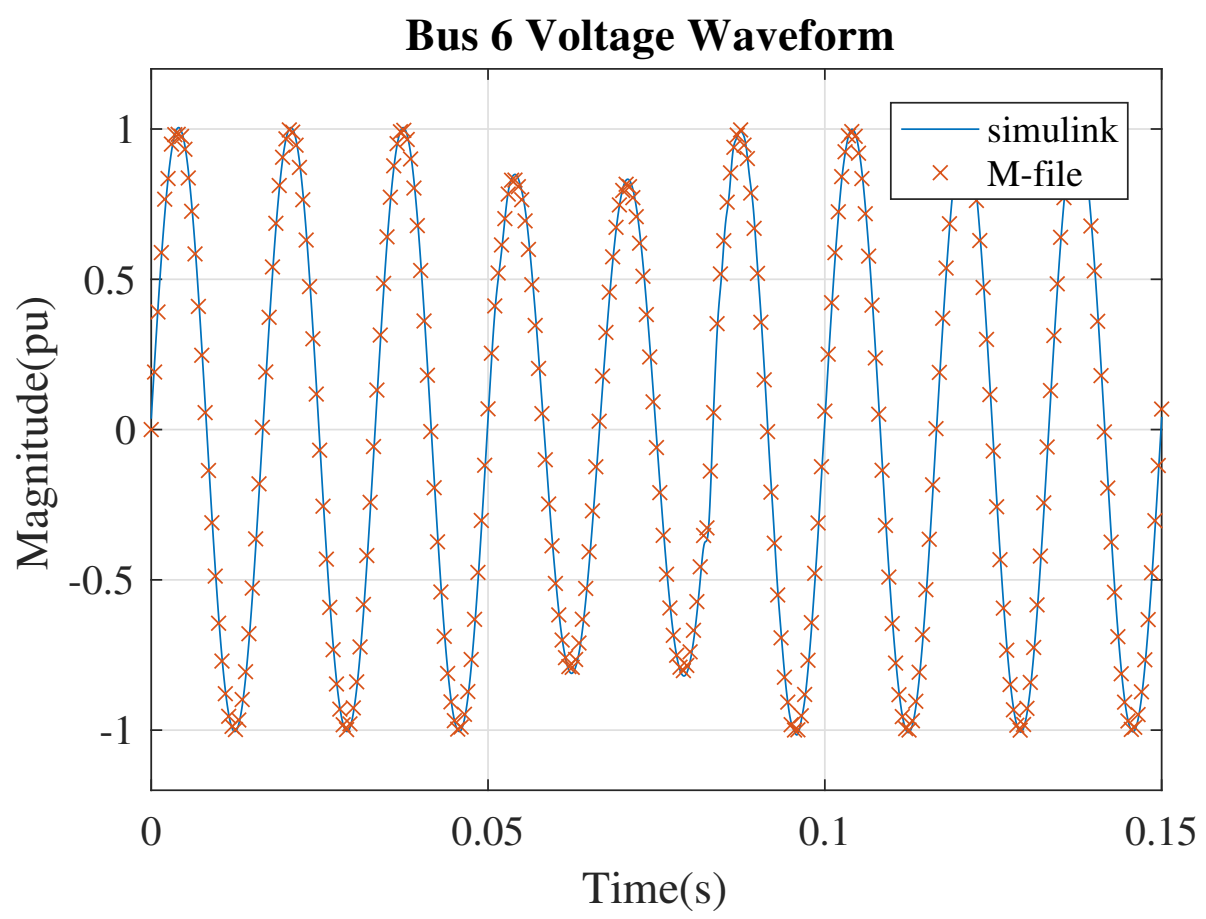

Figure 5.3: Voltage transient change at bus 6 
updated instantly, which leads to a larger accuracy index.

\subsubsection{Speed Performance}

The computation speed is illustrated in Table. 5.1. A simulation interval of $1 \mathrm{~s}$ is used in all tests listed in the Table 5.1, with the time step 10us. Simulink, as a professional program, requires more calculating resources. The ability to simulate a nonlinear system is weak. When a model contains an algebraic loop, Simulink uses a nonlinear solver at each time step to solve the algebraic loop. The solver performs iterations to determine the solution to the algebraic constraint, if there is one. As a result, the models with algebraic loops can run more slowly than the models without algebraic loops.

The system is partitioned into two subsystems to apply the proposed method. As seen in the table, there is no obvious advantage for the DD or NDD, compared with the original method. The reason is that when the matrix is extremely small, time spends on LU decomposition is almost the same for different sizes. Meanwhile, DD method introduces more multiplications and communication time during the whole simulation. As explained in Section. 4.3, when the communication time is larger than individual calculation time, the parallel calculation can only spend more CPU time. The broadcast function in spmd takes $4.16 \mathrm{~s}$ in total. If we discard the communication time, we can see the advantage of parallel calculation. In PDDL, some blocks are latent, therefore it is not necessary to do LU decomposition for all blocks. The time is reduced by around 6s, comparing with the DD method. 
Table 5.1: CPU time of IEEE 9 bus system

\begin{tabular}{|c||c|c|c|c|}
\hline & Variable number & sub-domain & Time(s) & Speed-Up \\
\hline Simulink & - & - & 25.30 & - \\
\hline Org & 138 & 2 & 18.16 & - \\
\hline DD & 138 & 2 & 19.53 & 0.93 \\
\hline PDDL & 138 & 2 & 13.60 & 1.33 \\
\hline NDD & 138 & 2 & 15.23 & 1.19 \\
\hline
\end{tabular}

\subsection{Case Study II: Medium-Scale Power System}

The second test-case is based on a medium-size power system. The IEEE 39-bus test system analyzed in this thesis is commonly known as "the 10-machine New-England Power System". This case is usually used to study simultaneous damping of local and inter-are modes in a system with a highly symmetrical structure. The system contains thirty-nine buses, thirty-four transmission lines, eighteen PQ loads and ten generators. Synchronous machines are modelled in detail, including voltage regulators and excitation systems.

This system has 628 state variables in total, 190 states for generators and 438 states for the grid. The original system is divided into two sub-domains, as shown in Figure. 4.9. After applying normal partitioning method, the first subsystem has 353 variables and the second subsystem has 263 variables. For the interface, there are 12 variables. This test case starts at a steady-state initial condition with the time step of 10us.

\subsubsection{Accuracy}

Figure. 5.4 shows the comparison of TS simulator and EMT simulator. TS simulator can only show the magnitude change briefly. High harmonic change is totally removed. 
However, EMT simulator and the proposed methods can show all the transient change. These transient changes are quite important for harmonic pollution analysis.

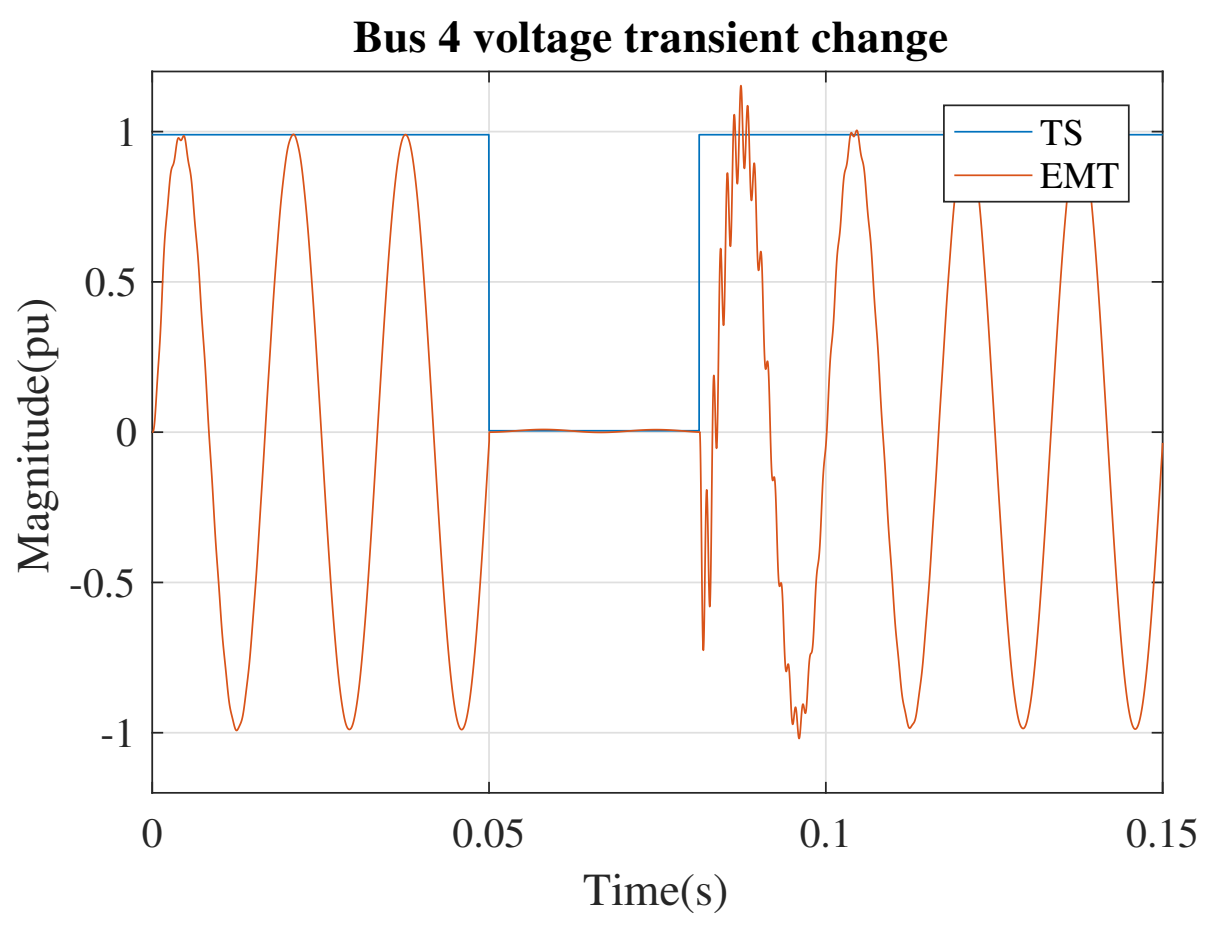

Figure 5.4: Voltage transient change at bus 4 by TS simulator

Figure. 5.5 and Figure. 5.6 show the transient change of voltage on bus 4 . To demonstrate the accuracy, the result of the proposed method is compared with the Simulink's result. The simulation was finished using the parameter in Table. B.3. We can see that when the power system is stable, the simulation results are exactly same. The introduced fault is the same as disturbance in Case I. After introducing disturbance, the voltage drops to almost zero rapidly. After the clearance of fault, the voltage oscillates severely. Fortunately, the power system takes one cycle to return back to be stable again. The accuracy index of DD and NDD algorithms are both around $6.82 \times 10^{-13}$. For the PDDL method, the accuracy index is around $3.6 \times 10^{-6}$. Since the simulation results are the same, in the following figure, results of DD, NDD, 


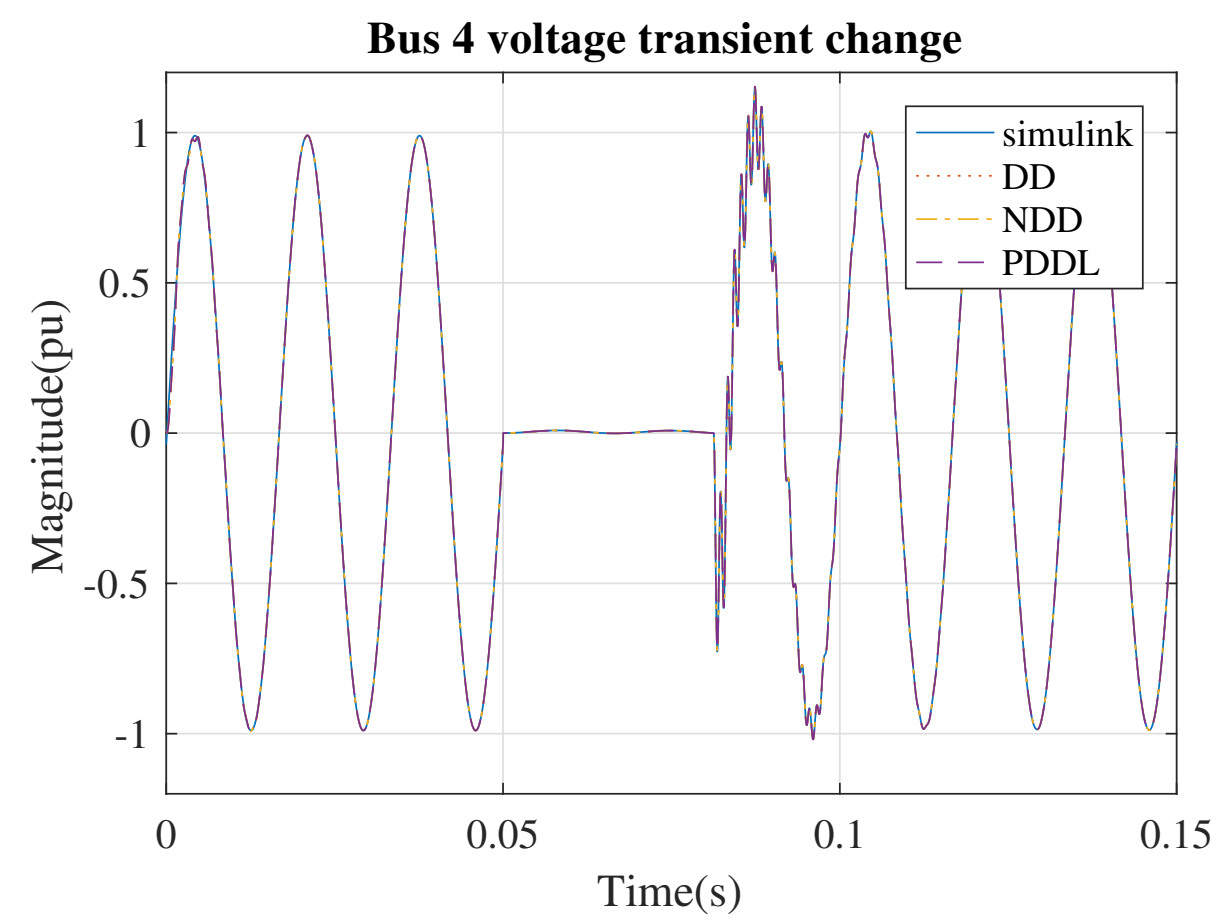

Figure 5.5: Voltage transient change at bus 4

and PDDL are combined into one group called 'M-file'.

Figure. 5.6 is the voltage transient change during the fault. This disturbance is equivalent to the short circuit between ground and transmission line, therefore the waveform is still sinusoidal but small. 


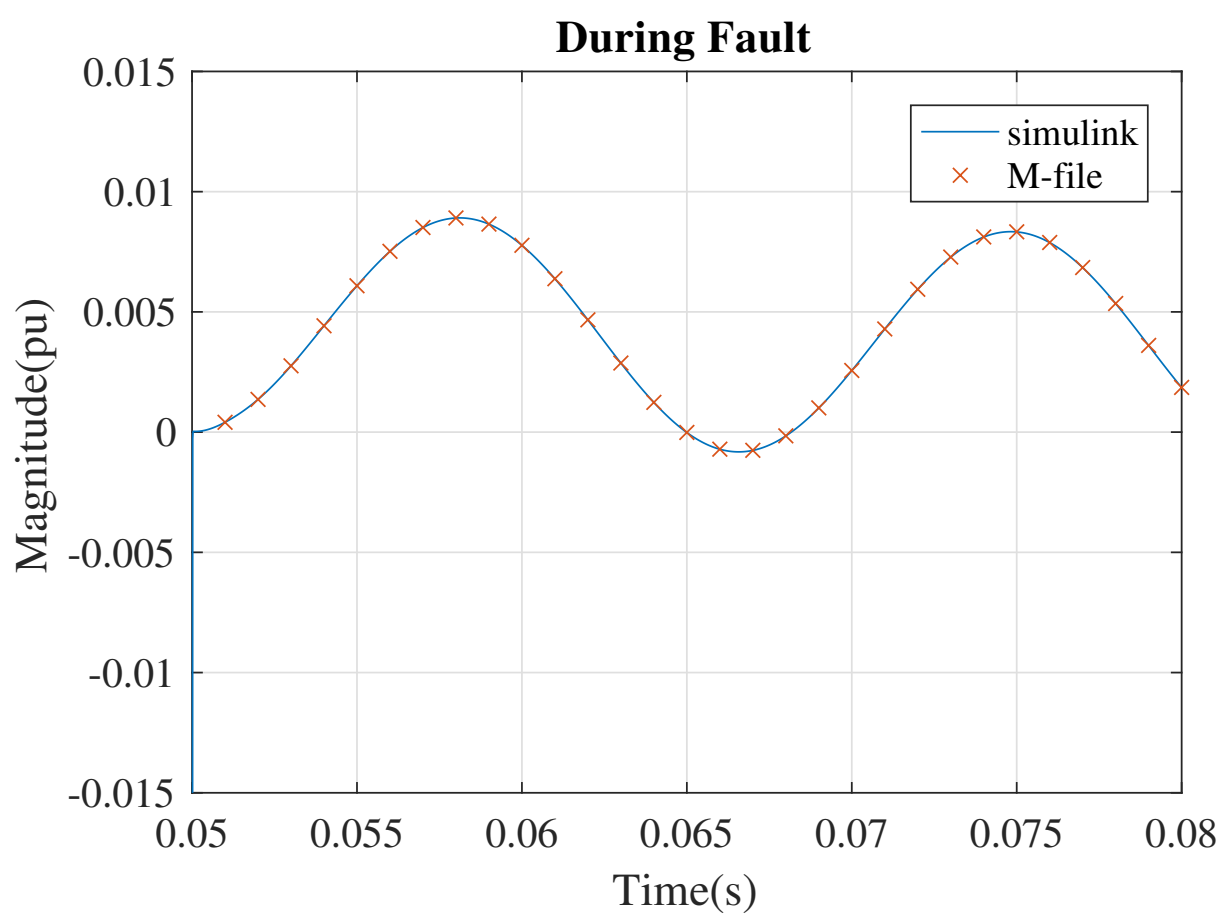

Figure 5.6: Voltage transient change of bus 4 during fault (zoom in)

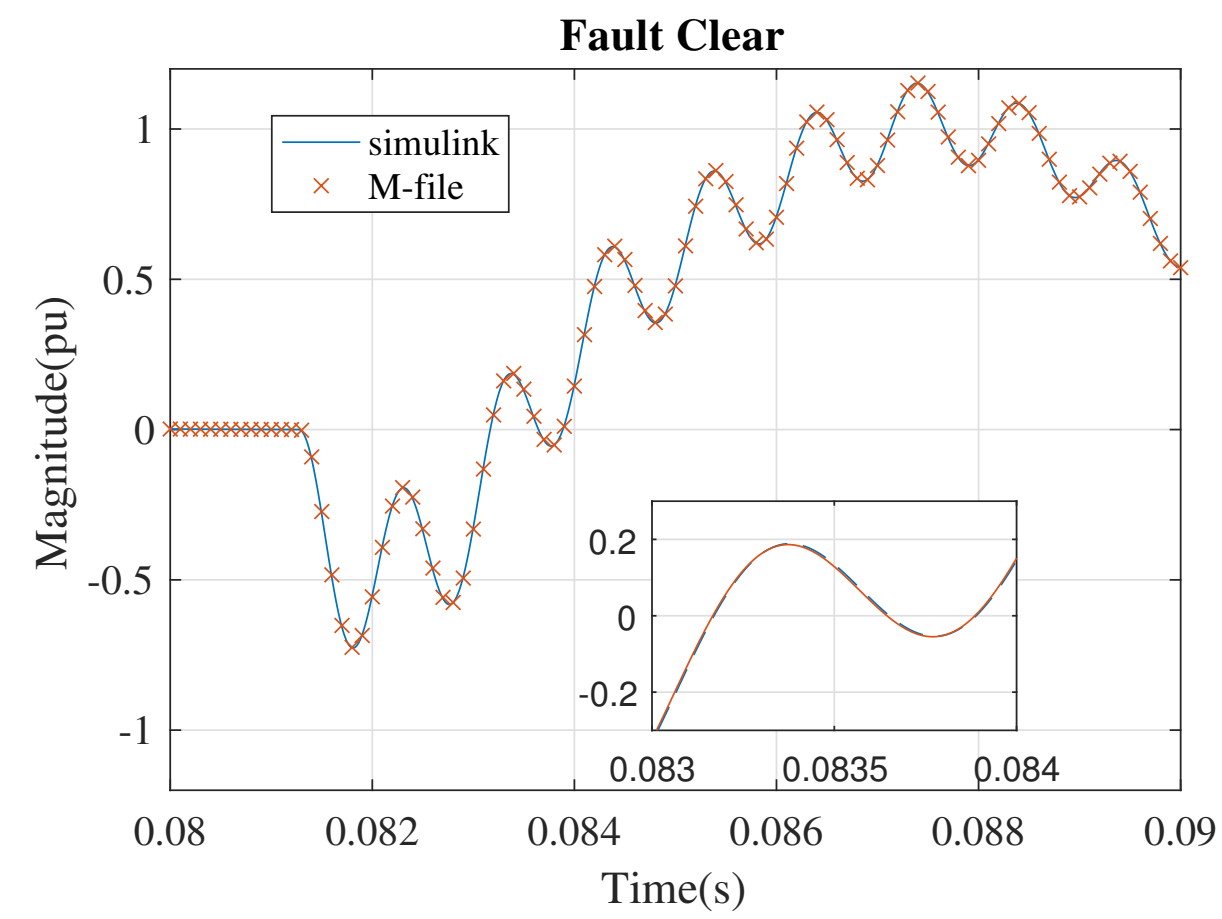

Figure 5.7: Voltage transient change of bus 4 after fault clear (zoom in) 


\subsubsection{Speed Performance}

Table. 5.2 summarizes the speed performance of IEEE 39 bus simulation. Algorithm DD, NDD and PDDL can speed up the simulation compared to algorithm Org. As the size of a power system increases, the calculation load of the LU decomposition exceeds the communication cost. In this case, the Jacobian matrix is separated into two similar sub-matrices, therefore the efficiency of parallel calculation is the highest. Comparing DD with original method, DD method speeds up around 1.61. The reason is that the saved time of $\mathrm{LU}$ decomposition is larger than the CPU time of communication and multiplication. Comparing DD with NDD, NDD does not do LU decomposition method for the grid subsystem but NDD does not show higher efficiency. For the DD method, LU decomposition of $A_{1}$ and $A_{2}$ runs in parallel. This is equivalent to do $\mathrm{LU}$ decomposition for just one matrix. Therefore, the $\mathrm{CPU}$ time for these two methods are similar. For the PDDL method, since less LU decomposition is needed, three seconds are saved.

Table 5.2: CPU Time of IEEE 39 bus system

\begin{tabular}{|c||c|c|c|c|}
\hline & Variable number & sub-domain & Time(s) & Speed-Up \\
\hline Simulink & - & - & 76.64 & - \\
\hline Org & 628 & 0 & 51.95 & - \\
\hline DD & 628 & 2 & 32.35 & 1.61 \\
\hline PDDL & 628 & 2 & 29.77 & 1.75 \\
\hline NDD & 628 & 2 & 31.74 & 1.63 \\
\hline
\end{tabular}

\subsection{Case Study III: Large-Scale Power System}

The third test-case is based on a large-size power system. The IEEE 118-bus test system analyzed in this thesis is a simple approximation of the electric power system 
in the midwest of United States. This case is usually used to study the transmission system. The system contains one hundred and eighteen buses, one hundred and eighty six transmission lines, ninety one PQ loads and fifty four generators. Synchronous machines are modelled in detail, including voltage regulator and excitation system.

This system has 2775 state variables in total, 1026 states for generators and 1749 states for the grid. After applying DD methods, the first subsystem has 1587 variables and the second subsystem has 756 variables. For the interface, there are 432 variables. The original system is divided into two sub-domains, as shown in Figure. B.3. This test case starts at steady state initial condition with time step equals 10us.

\subsubsection{Accuracy}

Figure. 5.8 shows the steady-state voltage on bus 17 using the proposed methods and Matlab/Simulink. The result of DD and NDD are combined into one group called 'M-file'. As seen, all methods are in good agreements. The accuracy index of DD and NDD are both $6.98 \times 10^{-7}$. Small difference happens during the disturbance. Latency method cannot be applied to these system due to the similar time constant of each subsystem.

In the following Figure. 5.9, Figure. 5.10 and Figure. 5.11, a fault is added to bus 16, which is adjacent to bus 17 . Since the fault is not directly applied to the observed bus, the voltage does not drop to zero. When the fault happens, the voltage is around 0 , therefore the transient change is smooth. When the fault is removed, the bus voltage drop to $-0.8 \mathrm{pu}$ suddenly due to the sudden change of the power system. 


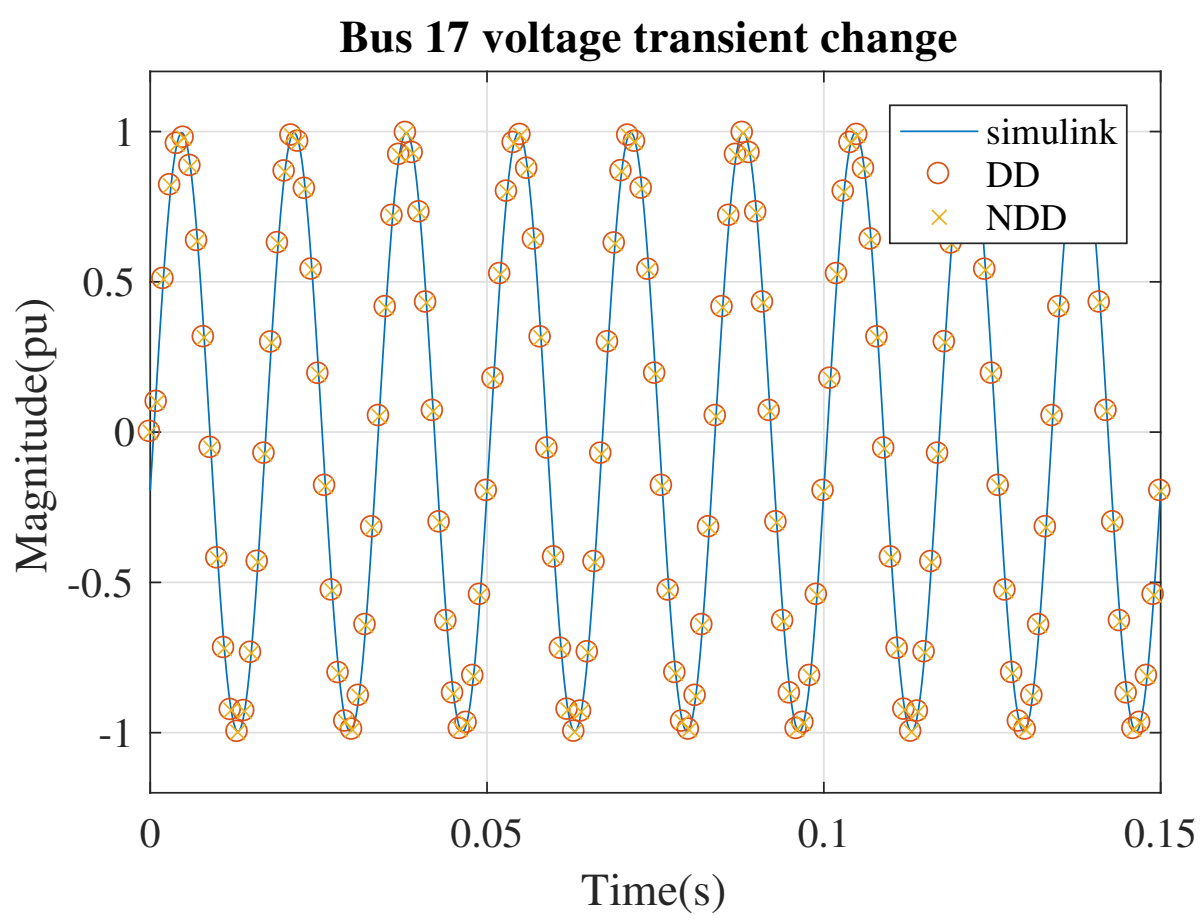

Figure 5.8: Steady state voltage on bus 17

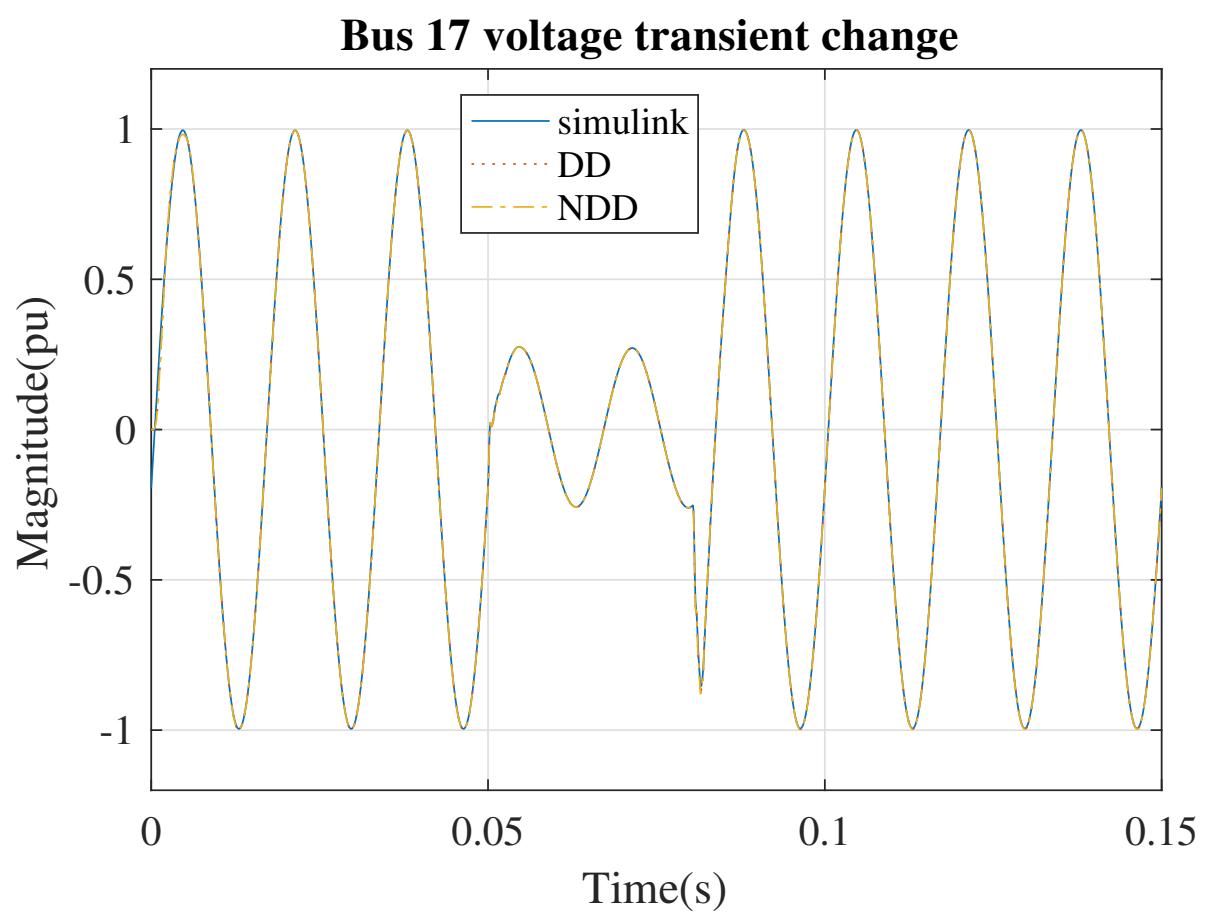

Figure 5.9: Transient voltage on bus 17 


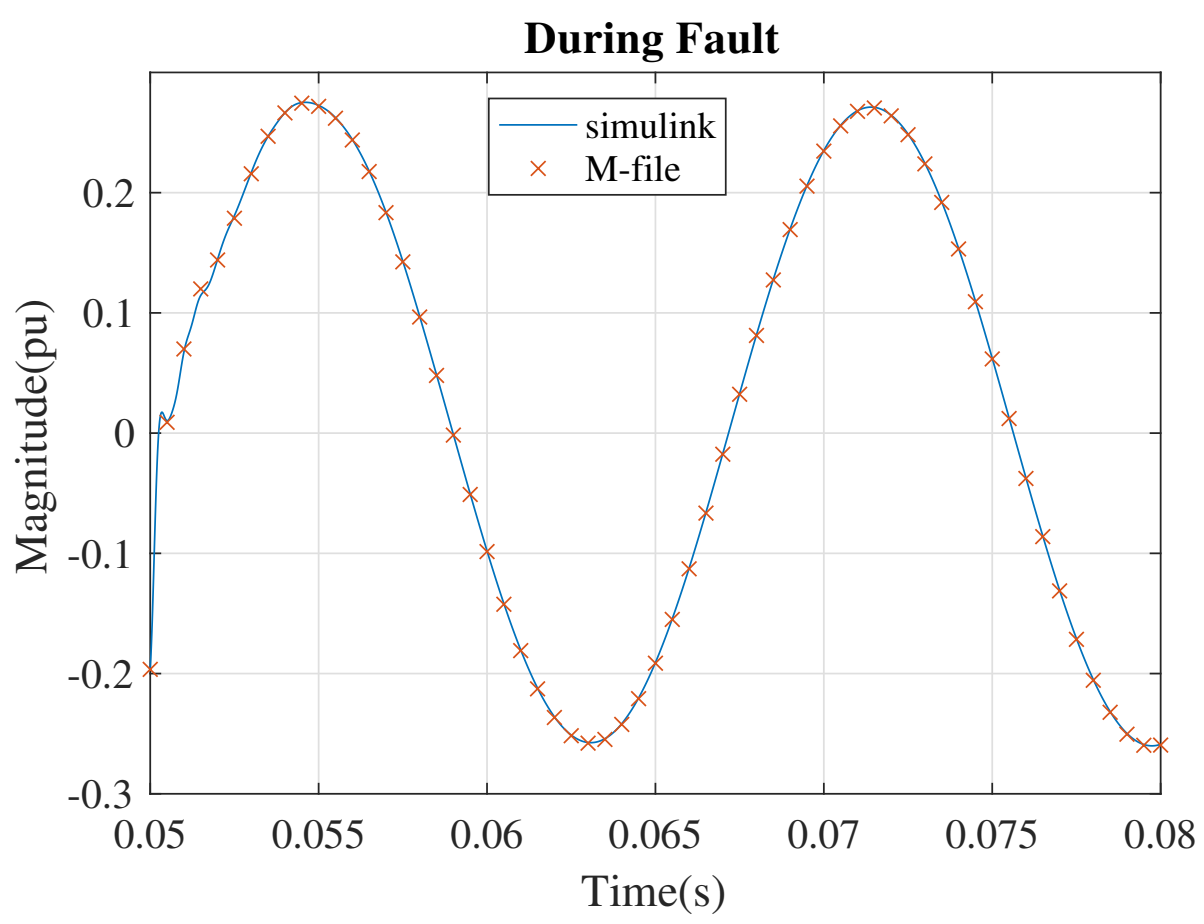

Figure 5.10: Voltage transient change of bus 17 during fault

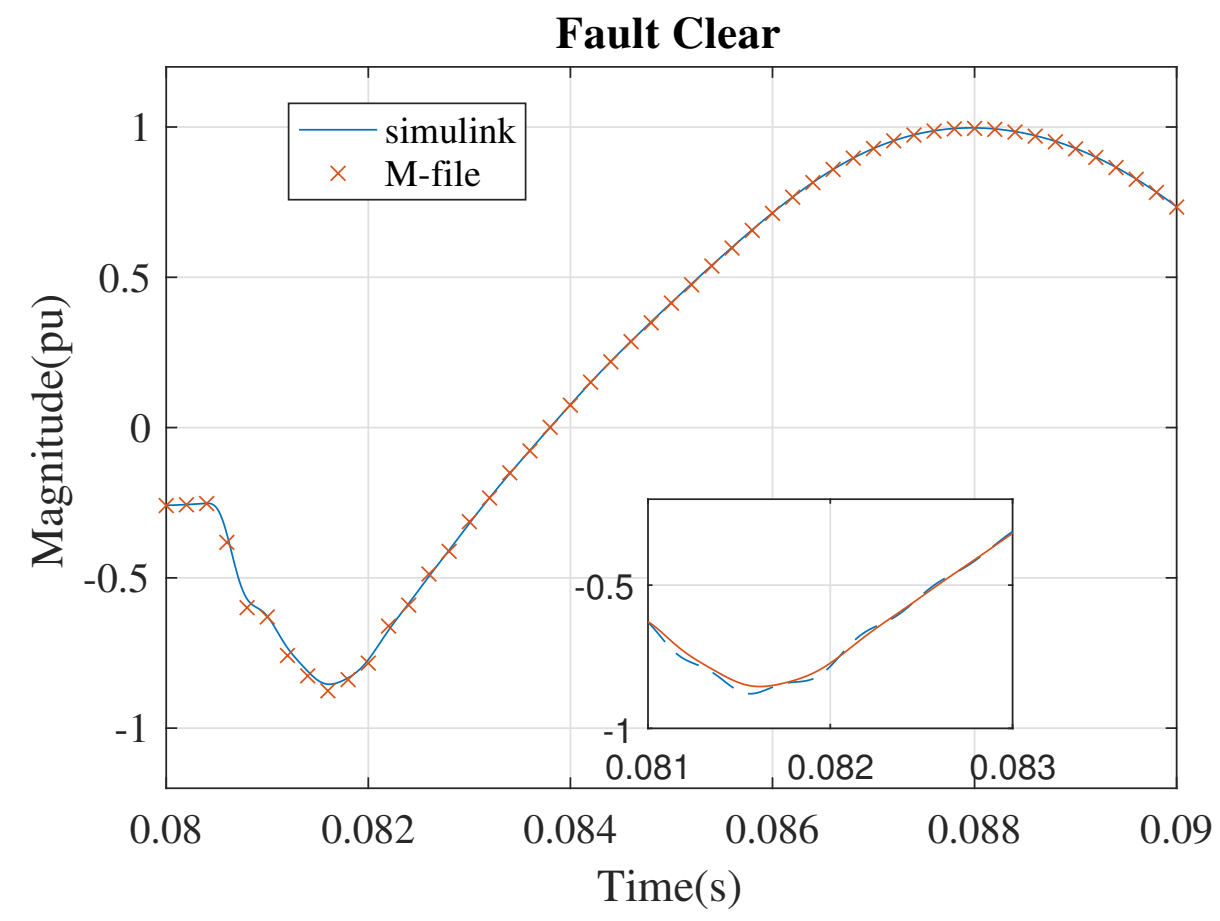

Figure 5.11: Voltage transient change of bus 17 after fault clear 


\subsubsection{Speed Performance}

Table. 5.3 compares the CPU cost between the proposed methods and the original method. The DD method can still improve the efficiency of simulation while the efficiency is almost the same as that in IEEE 39 bus system, which proves the correctness of this proposed method. To explore the detail CPU time distribution, the time spends on calculating Schur complement matrix is measured. Schur complement matrix calculation accounts for the largest (40\%) during the simulation. With the increasing size of the system, the extra calculation cannot be ignored. It may affect the efficiency. Fortunately, DD method can still handle this example. For the NDD method, the improvement is obvious, due to the pre-calculated matrices. The precalculated matrices, as the major part of Schur complement matrix, can help saving much time.

Table 5.3: CPU Time of IEEE 118 bus system

\begin{tabular}{|c||c|c|c|c|}
\hline & Variable number & sub-domain & Time(s) & Speed Up \\
\hline Simulink & - & - & 180.17 & - \\
\hline Org & 2775 & 0 & 114.78 & - \\
\hline DD & 2775 & 2 & 70.56 & 1.63 \\
\hline NDD & 2775 & 2 & 53.7 & 2.13 \\
\hline
\end{tabular}




\subsection{Case Study IV: Large-Scale Power System}

The fourth test-case is based on another large-size power system. The IEEE 2869bus test system is analyzed in this thesis. The system contains 2869 buses, 4582 transmission lines, $1491 \mathrm{PQ}$ loads and 510 generators. This system is too large for Matlab/Simulink, therefore the transient change by simulink cannot be finished. The steady state voltage can be obtained from the MATPOWER testcase. MATPOWER is Matlab toolbox which does power flow calculation and can obtain the steady state voltage. In power flow calculation, the total variables are 11476 and 5227 of them are unknowns.

In detailed modelling, this system has 43773 state variables before decomposition. After applying DD methods, the first subsystem has 31023 variables and the second subsystem has 7140 variables. There are 4080 variables in the interface. The original system is too large to be shared in this thesis. The original data for the transmission lines and loads can be found in the testcase of MATPOWER [66].

\subsubsection{Accuracy}

Since we can only obtain the voltage magnitude and phase angle from MATPOWER, the magnitudes of some buses are compared in this example. The comparison of transient change will be finished by other program like PSCAD in the future. Therefore only power flow calculations (steady state voltages) are compared in this section.

Table. 5.4 shows the power flow calculation of IEEE 2869 bus system. The results are shown in per unit value. The MATPOWER column means the result from MATPOWER and Matlab Script means the result from Matlab script. The magnitude shows good agreement.

Figure. 5.12 shows the transient simulation result. The disturbance is applied 
Table 5.4: Examples of IEEE 2869 bus system power flow calculation

\begin{tabular}{|c||c|c|}
\hline Bus No. & MATPOWER $(\mathrm{pu})$ & MATLAB Script(pu) \\
\hline 32 & 1.0062 & 1.00616 \\
\hline 976 & 1.0298 & 1.02972 \\
\hline 1173 & 1.0261 & 1.02609 \\
\hline 2550 & 1.0509 & 1.05098 \\
\hline
\end{tabular}

to bus 21 which is close to bus 6163 . Org is original method that solve the power system as a whole. There is some dynamic change when the fault happens. Original method is exactly the same as DD method, cause the only difference is the order of unknowns. The results are expected to be the same.

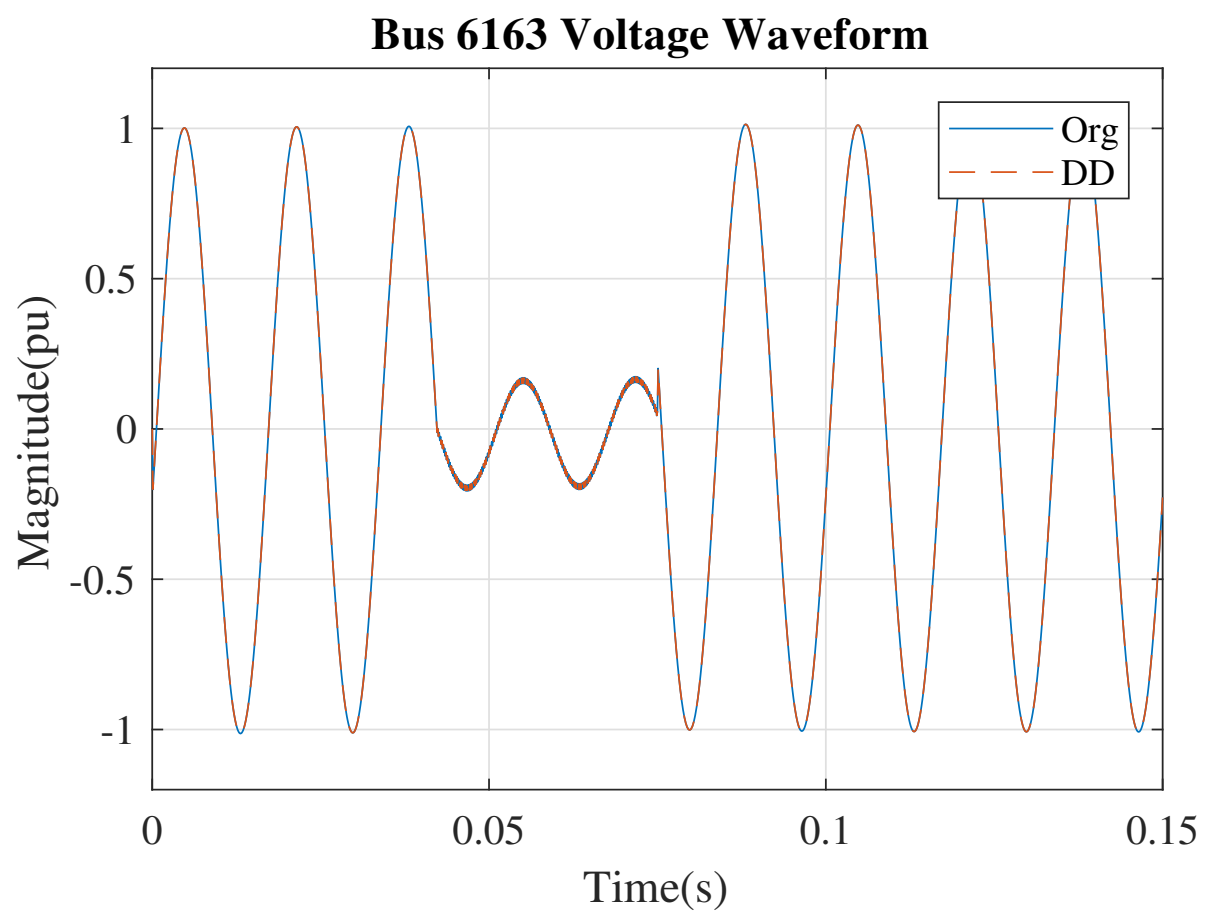

Figure 5.12: Voltage transient change of bus 6163 


\subsubsection{Speed Performance}

In this subsection, detailed modelling is used. Synchronous machines are modelled in detail, including excitation system. Table. 5.5 shows the comparison of the CPU cost used in the simulation of proposed methods and the simulation of original method. In this super-large power system, the original DD method cannot improve the efficiency of simulation. Because of the extra multiplication for the Schur complement matrix, the explicit calculation cannot be accepted. In this example, the Schur matrix is $\boldsymbol{S} \in \mathbb{R}^{4800 \times 4800}$. For the explicit calculation of Schur matrix, the multiplication of

three matrices $\left(\boldsymbol{D} \in \mathbb{R}^{N_{s} \times N_{i}}, \boldsymbol{A} \in \mathbb{R}^{N_{i} \times N_{i}}, \boldsymbol{C} \in \mathbb{R}^{N_{i} \times N_{s}}\right.$ ) may needs hundred million calculations. However, for the original method, the multiplication happens between a square matrix and a matrix with one column. This, as plenty of DD method, can be fixed by binary link formulation. This is a method which separates system based on node instead of branch. If node tearing is applied to this power system, $\boldsymbol{C}$ matrices are guaranteed to includes only \pm 1 and 0 . During each iteration, nonzeros are static. When the matrix size is around 30000, multiplications can be sped up by around 3.71 [67]. In other words, the total simulation speed can be doubled. Due to the characteristic of NDD, the majority calculation can be avoided, then the efficiency is still improved.

Table 5.5: CPU Time of IEEE 2869 bus system

\begin{tabular}{|c||c|c|c|c|}
\hline & Variable number & sub-domain & Time(s) & Speed-Up \\
\hline Org & 43773 & - & 4624.7 & - \\
\hline DD & 43773 & 2 & 5864.2 & 0.788 \\
\hline NDD & 43773 & 2 & 3778.4 & 1.22 \\
\hline
\end{tabular}




\subsection{Speed-Up Analysis}

Time complexity of each algorithm will be discussed in detail in this section. No matter what method is used, at the beginning, initialization must be processed. Initialization process includes generating $\boldsymbol{G}$ matrix and $\boldsymbol{C}$ matrix for the whole system and power flow calculation. All these commands only run once at beginning and process time (hundred milliseconds) can be ignored by comparing with NR iteration loop processing time (hundred seconds). Newton method can be divided into three parts, including Jacobian Matrix generation, LU decomposition and Forward/Backward substitution. Jacobian Matrix is generated based on partial derivation of non-linear item. The partial derivations of each function are stored in memory. All the algorithms need calculating Jacobian matrix with the same CPU load. Once Jacobian matrix $\boldsymbol{J} \in \mathbb{R}^{n \times n}$ is obtained, different methods can be applied to solve (4.7).

For a sparse matrix, the time complexity for LU decomposition is shown in (5.3).

$$
\Theta=\Theta\left(n^{\alpha}\right)
$$

where $\alpha$ depends on the density, which usually ranges from 1.1 to 2 .

If the domain decomposition method is applied, the computational time can be reduced. At first, we assume the system is divided into $p$ subdomains equally, the processing complexity will become:

$$
\Theta=\Theta\left(p\left(\frac{n}{p}\right)^{\alpha}\right)
$$

where $n$ is matrix size of each subdomain. After applying parallel calculation by $m$ 
processors, the processing complexity will become:

$$
\Theta=\Theta\left(\frac{p}{m}\left(\frac{n}{p}\right)^{\alpha}\right)
$$

When the original system is not divided equally, or the subsystems are not balanced. Processors with small matrices need to wait for processors with large matrices. The processing complexity will become:

$$
\Theta=\Theta\left(\frac{p}{m}\left(\max \left(n^{\prime}\right)^{\alpha}\right)\right)
$$

where $n^{\prime}$ is the size of matrix for each subdomain. In general, there are three time Newton iterations in average for each time step, the total CPU time is

$$
\Theta(n)=3 * H * \Theta\left(\frac{p}{m}\left(\max \left(n^{\prime}\right)^{\alpha}\right)\right.
$$

where $\mathrm{H}$ is the number of time-step.

Calculating interface variables is another dominating calculation during simulation. Eq. 4.11 and (4.12) are the accumulation of a sparse matrix multiplied by a dense matrix. For the traditional DD method, if $n_{i}$ is the number of variables connecting to subsystem $i, p$ is the total number of subdomains, then the total number of accumulations is:

$$
\sum_{i=1}^{p} n_{i}^{2}
$$

Since there are some zeros in the sparse $\boldsymbol{D}_{i}$ matrix, the total number of multiplications to construct Schur matrix in (4.11) will be equal to the number of accumulations multiplied by a coefficient. The coefficient $\left(k_{0}\right)$ represents the nonzeros per row in $\boldsymbol{D}_{i}$. 
Therefore, the total number of multiplications will be:

$$
k_{0} \sum_{i=1}^{p} n_{i}^{2}
$$

For binary link formulation, 4.11) and 4.12 can be solved with simple permutation and accumulate operations. No more multiplications is needed. Therefore, the time complexity is:

$$
\Theta(0)
$$

If the latency is also applied to DD, some sub-systems stop LU decomposition. In parallel calculation, latency cannot improve efficiency a lot. Only when the largest subdomain is latent, the advantage of latency can be exploited.

\subsection{Analysis of Multi-cores Simulation}

Based on the previous time complexity analysis, to explore the improvement of parallel calculation in multicores, IEEE 2869 bus system are running on three cores, four cores, six cores, and eight cores, respectively. There are 510 generators in this system, therefore it is easy to divide them into several groups. Figure. 5.13 shows the experiment result corresponding to analysis in Section. 5.7. Blue line is the theory value, red line is the experiment value. Experiment includes communication time and any other extra calculation. The comparison shows that when the core number is larger than 6 , the parallel calculation cannot help to improve efficiency. The time complexity for communication between each processors is:

$$
\Theta\left(n^{2}\right)
$$




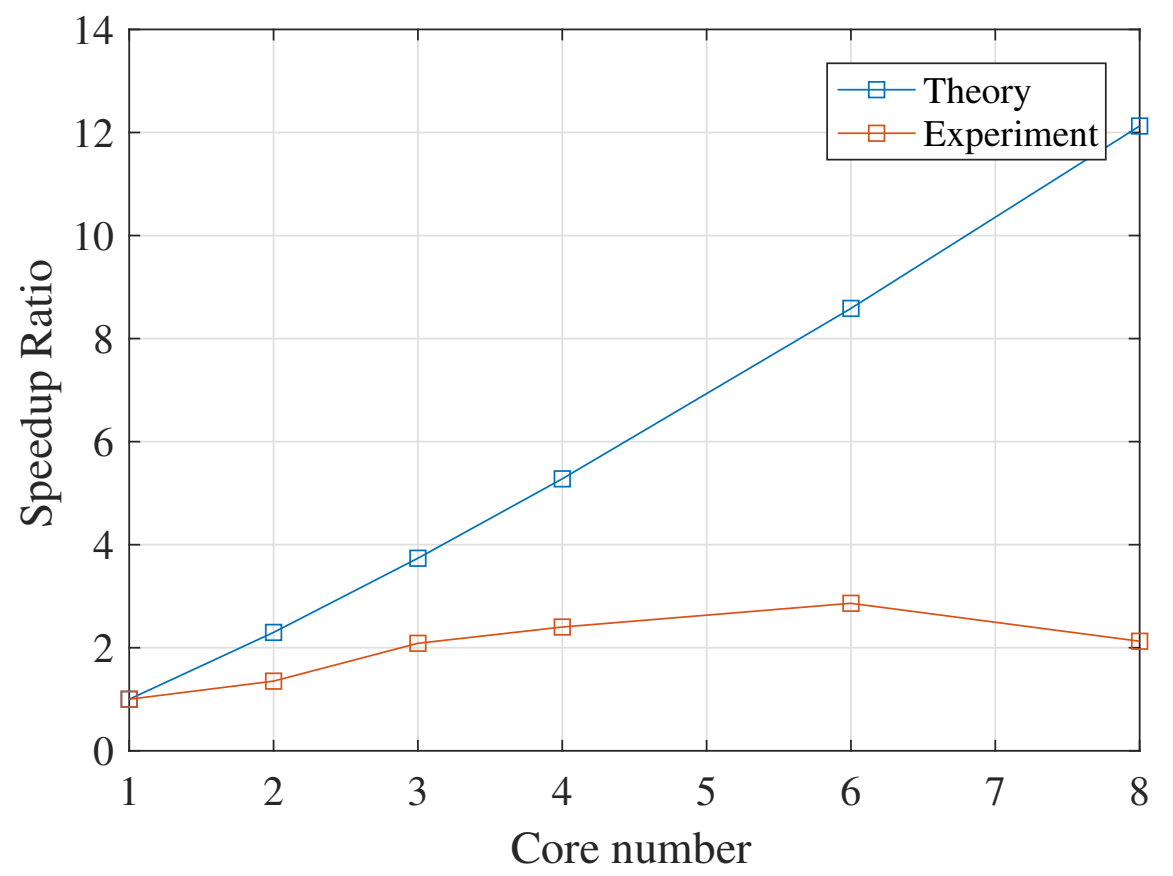

Figure 5.13: Speedup of algorithm DD

With the increasing of processors, communication processes take more time. Four or six cores are enough to obtain the best performance. 


\section{Chapter 6}

\section{Conclusion and Future Work}

\subsection{Conclusions}

In this thesis, parallel domain decomposition methods integrating latency method for power system simulation have been presented. Conventional simulators are computationally expensive. This is mainly due to the following reasons: (1). The model is running as a whole. All the equations are solved simultaneously; (2). Modelling method is complex, unnecessary calculations are included. Numerical results have demonstrated that the proposed method can address these issues without sacrificing the accuracy. Regarding the power system simulation methods tested in this thesis, the following conclusions are summarized.

- These proposed methods accelerate the simulation speed in two ways. Firstly, the procedure reduces time on numerical calculation by splitting the whole system and discarding useless calculations (factorizations, updates). Secondly, parallelization is applied to DD methods.

- The proposed methods keep the same accuracy as traditional EMT method due to the similar modelling method and similar solver. 
- DD has the good potential to reduce the CPU time. It can be further improved by applying binary link formulation to large power system.

- Compared with the TS simulator, the proposed algorithm is more accurate, as the original system is modelled in time domain. High frequency components can be seen in the simulation results by the proposed algorithm. Owing to the three phase modelling, simulation of each phase can also be seen in the result.

- Compared with the traditional EMT simulator, MNA method provides a more convenient and scientific way to do power system modelling.

- The partitioning method also has large impact on the CPU time because the number of interface variables and characteristic of each subdomain. Balanced partition could lead to the best performance. With the help of new partitioning method, simulation efficiency can be further improved by pre-calculating invariant matrices.

- Increasing number of processors can help to improve the efficiency. The efficiency will come to a saturation region with the increasing of core number due to more communication overhead. This saturation region can be avoided by using shared memory software.

\subsection{Future Work}

For future research, the following directions can be considered:

- Binary link formulations can be applied to power system simulation. Binary link formulations is a proved method that can reduce complexity of DD method from $\Theta\left(L^{2}\right)$ (traditional DD method) to $\Theta(L)$ (PvB method), where $\mathrm{L}$ is the number of links. 
- Shared-memory software like OpenMP can be used to do parallel calculation, therefore, the communication time can be saved. The algorithm is still the same, calculation load cannot be reduced. However, with the decrease of communication time, the efficiency will increase when multi-cores are used.

- Large scale power system needs finishing in some very professional commercial product to obtain the reference value. Simulink cannot afford the calculation resource of large power system. After obtaining the simulation result of transient change, accuracy performance of the proposed method in large power system can be verified. 


\section{List of References}

[1] E. Rajan and S. Amrutha, "Synchronverter based hvdc transmission," in 2017 Innovations in Power and Advanced Computing Technologies (i-PACT), pp. 1-6, April 2017.

[2] T. J. Hammons, V. F. Lescale, K. Uecker, M. Haeusler, D. Retzmann, K. Staschus, and S. Lepy, "State of the art in ultrahigh-voltage transmission," Proceedings of the IEEE, vol. 100, pp. 360-390, Feb 2012.

[3] J. Cao and J. Cai, "Hvdc in china," in 2013 HVDC and FACTS Conference, Palo Alto, CA, USA, 2013.

[4] B. Stott, "Power system dynamic response calculations," Proceedings of the IEEE, vol. 67, pp. 219-241, Feb 1979.

[5] W. S. Meyer and H. W. Dommel, "Numerical modelling of frequency-dependent transmission-line parameters in an electromagnetic transients program," IEEE Transactions on Power Apparatus and Systems, vol. PAS-93, pp. 1401-1409, Sept 1974.

[6] H. W. Dommel, "Digital computer solution of electromagnetic transients in single-and multiphase networks," IEEE Transactions on Power Apparatus and Systems, vol. PAS-88, pp. 388-399, April 1969.

[7] H. W. Dommel and W. S. Meyer, "Computation of electromagnetic transients," Proceedings of the IEEE, vol. 62, pp. 983-993, July 1974.

[8] H. Dommel, Electromagnetic Transients Program: Reference Manual : (EMTP Theory Book). Bonneville Power Administration, 1986.

[9] D. P. Koester, S. Ranka, and G. C. Fox, "Power systems transient stability - a grand computing challenge," 1992. 
[10] A. Atputharajah and T. K. Saha, "Power system blackouts - literature review," in 2009 International Conference on Industrial and Information Systems (ICIIS), pp. 460-465, Dec 2009.

[11] B. Sabeeh and C. Gan, "Power system frequency stability and control: Survey," in International Journal of Applied Engineering Research, pp. 5688-5695, Nov 2016.

[12] R. Dunlop, "Proposed terms definitions for power system stability," IEEE Transactions on Power Apparatus and Systems, vol. PAS-101, pp. 1894-1898, July 1982.

[13] P. Kundur, J. Paserba, V. Ajjarapu, G. Andersson, A. Bose, C. Canizares, N. Hatziargyriou, D. Hill, A. Stankovic, C. Taylor, T. V. Cutsem, and V. Vittal, "Definition and classification of power system stability ieee/cigre joint task force on stability terms and definitions," IEEE Transactions on Power Systems, vol. 19, pp. 1387-1401, Aug 2004.

[14] J. Mahseredjian, "Computation of power system transients: overview and challenges," in 2007 IEEE Power Engineering Society General Meeting, pp. 1-7, June 2007.

[15] L. Dube and H. Dommel, "Simulation of control systems in an electromagnetic transients program with tacs," in Proc. IEEE Power Industry Computer Applications Conf, pp. 266-271, Nov 1977.

[16] K.S.Turner, M.D.Heffernan, C.P.Arnold, and J.Arrillaga, "Computation of acdc system disturbances. part i, ii and iii," IEEE Power Engineering Review, vol. PER-1, 1981.

[17] J. Reeve and R. Adapa, "A new approach to dynamic analysis of ac networks incorporating detailed modeling of dc systems. i. principles and implementation," IEEE Transactions on Power Delivery, vol. 3, pp. 2005-2011, Oct 1988.

[18] H. T. Su, L. A. Snider, K. W. Chan, and B. Zhou, "A new approach for integration of two distinct types of numerical simulator," in Proc. International Conference on Power Systems Transients-IPST, Oct 2003.

[19] X. Wang, P. Wilson, and D. Woodford, "Interfacing transient stability program to emtdc program," in Proceedings. International Conference on Power System Technology, vol. 2, pp. 1264-1269 vol.2, 2002. 
[20] V. Jalili-Marandi, V. Dinavahi, K. Strunz, J. A. Martinez, and A. Ramirez, "Interfacing techniques for transient stability and electromagnetic transient programs ieee task force on interfacing techniques for simulation tools," IEEE Transactions on Power Delivery, vol. 24, pp. 2385-2395, Oct 2009.

[21] H. Inabe, T. Futada, H. Horii, and K. Inomae, "Development of an instantaneous and phasor analysis combined type real-time digital power system simulator," in International Conference on Power Systems Transients, April 2003.

[22] W. Liwei, D. Z. Fang, and T. S. Chung, "New techniques for enhancing accuracy of emtp/tsp hybrid simulation algorithm," in 2004 IEEE International Conference on Electric Utility Deregulation, Restructuring and Power Technologies. Proceedings, vol. 2, pp. 734-739 Vol.2, April 2004.

[23] A. A. van der Meer, M. Gibescu, M. A. M. M. van der Meijden, W. L. Kling, and J. A. Ferreira, "Advanced hybrid transient stability and emt simulation for vschvdc systems," IEEE Transactions on Power Delivery, vol. 30, pp. 1057-1066, June 2015.

[24] T. Fang, Y. Chengyan, W. Zhongxi, and Z. Xiaoxin, "Realization of electromechanical transient and electromagnetic transient real time hybrid simulation in power system," in 2005 IEEE/PES Transmission Distribution Conference Exposition: Asia and Pacific, pp. 1-6, 2005.

[25] H. T. Su, K. W. Chan, L. A. Snider, T. S. Chung, and D. Z. Fang, "Recent advancements in electromagnetic and electromechanical hybrid simulation," in 2004 International Conference on Power System Technology, 2004. PowerCon 2004., vol. 2, pp. 1479-1484 Vol.2, Nov 2004.

[26] H. T. Su, K. W. Chan, and L. A. Snider, "Parallel interaction protocol for electromagnetic and electromechanical hybrid simulation," IEE Proceedings Generation, Transmission and Distribution, vol. 152, pp. 406-414, May 2005.

[27] A. S. Morched and V. Brandwajn, "Transmission network equivalents for electromagnetic transients studies," IEEE Power Engineering Review, vol. PER-3, pp. 31-32, Sept 1983.

[28] H. Su, K. K. W. Chan, and L. A. Snider, "Interfacing an electromagnetic svc model into the transient stability simulation," in Proceedings. International Conference on Power System Technology, vol. 3, pp. 1568-1572 vol.3, 2002. 
[29] X. Wang, P. Wilson, and D. Woodford, "Interfacing transient stability program to emtdc program," in Proceedings. International Conference on Power System Technology, vol. 2, pp. 1264-1269 vol.2, 2002.

[30] Q. Huang and V. Vittal, "Application of electromagnetic transient-transient stability hybrid simulation to fidvr study," IEEE Transactions on Power Systems, vol. 31, pp. 2634-2646, July 2016.

[31] D. Shu, X. Xie, Q. Jiang, Q. Huang, and C. Zhang, "A novel interfacing technique for distributed hybrid simulations combining emt and transient stability models," IEEE Transactions on Power Delivery, vol. 33, pp. 130-140, Feb 2018.

[32] P. Kundur, N. Balu, and M. Lauby, Power system stability and control. EPRI power system engineering series, McGraw-Hill, 1994.

[33] PSCAD, "Ieee 09 bus system," PSCAD, December 2014.

[34] F. Plumier, P. Aristidou, C. Geuzaine, and T. V. Cutsem, "Co-simulation of electromagnetic transients and phasor models: A relaxation approach," IEEE Transactions on Power Delivery, vol. 31, pp. 2360-2369, Oct 2016.

[35] Y. Hu, W. Wu, B. Zhang, and Q. Guo, "Development of an rtds-tsa hybrid transient simulation platform with frequency dependent network equivalents," in IEEE PES ISGT Europe 2013, pp. 1-5, Oct 2013.

[36] B. Porkar, M. Vakilian, R. Feuillet, M. Ghassemi, and A. Akhavan, "Multi-port frequency-dependent network equivalent for electromagnetic transient studies," in Proceedings of the 37th Annual North American Power Symposium, 2005., pp. 287-295, Oct 2005.

[37] D. Shu, H. Yang, X. Xie, X. Ye, S. Yu, and Q. Jiang, "A novel dynamic phasor based interface models for hybrid simulations of emt and transient stability models," in 2017 IEEE Power Energy Society General Meeting, pp. 1-5, July 2017.

[38] F. J. Plumier, C. Geuzaine, and T. V. Cutsem, "On the convergence of relaxation schemes to couple phasor-mode and electromagnetic transients simulations," in 2014 IEEE PES General Meeting - Conference Exposition, pp. 1-5, July 2014.

[39] A. Meer, M. Gibescu, M. Meijden, W. Kling, and J. Ferreira, "Advanced hybrid transient stability and emt simulation for vsc-hvdc systems," IEEE Transactions on Power Delivery, vol. 30, pp. 1057-1066, 062015. 
[40] Q. Huang and V. Vittal, "Openhybridsim: An open source tool for emt and phasor domain hybrid simulation," in 2016 IEEE Power and Energy Society General Meeting (PESGM), pp. 1-5, July 2016.

[41] S. Abhyankar and A. J. Flueck, "An implicitly-coupled solution approach for combined electromechanical and electromagnetic transients simulation," in 2012 IEEE Power and Energy Society General Meeting, pp. 1-8, July 2012.

[42] X. Zhang, A. J. Flueck, and S. Abhyankar, "Implicitly coupled electromechanical and electromagnetic transient analysis using a frequency-dependent network equivalent," IEEE Transactions on Power Delivery, vol. 32, pp. 1262-1269, June 2017.

[43] N. Lu, Z. T. Taylor, D. P. Chassin, R. Guttromson, and S. Studham, "Parallel computing environments and methods for power distribution system simulation," in IEEE Power Engineering Society General Meeting, 2005, pp. 215-220 Vol. 1, June 2005.

[44] F. L. Alvarado, D. C. Yu, and R. Betancourt, "Partitioned sparse a/sup -1/ methods (power systems)," IEEE Transactions on Power Systems, vol. 5, pp. 452-459, May 1990.

[45] B. Sullivan, J. Shi, M. Mazzola, and B. Saravi, "Faster-than-real-time power system transient stability simulation using parallel general norton with multiport equivalent (pgnme)," in 2017 IEEE Power Energy Society General Meeting, pp. 1-5, July 2017.

[46] J. Shu, W. Xue, and W. Zheng, "A parallel transient stability simulation for power systems," IEEE Transactions on Power Systems, vol. 20, pp. 1709-1717, Nov 2005.

[47] G. Gan, Z. Zhu, G. Geng, and Q. Jiang, "An efficient parallel sequential approach for transient stability emergency control of large scale power system," IEEE Transactions on Power Systems, pp. 1-1, 2018.

[48] A. Iserles, A First Course in the Numerical Analysis of Differential Equations. Cambridge Texts in Applied Mathematics, Cambridge University Press, 1996.

[49] R. H. Park, "Two-reaction theory of synchronous machines generalized method of analysis-part i," Transactions of the American Institute of Electrical Engineers, vol. 48, pp. 716-727, July 1929. 
[50] IEEE, "Ieee recommended practice for excitation system models for power system stability studies," IEEE Std 421.5-2005 (Revision of IEEE Std 421.5-1992), pp. 1-93, April 2006.

[51] T. Shanker and R. K. Singh, "Wind energy conversion system: A review," in 2012 Students Conference on Engineering and Systems, pp. 1-6, March 2012.

[52] M. Chinchilla, S. Arnaltes, and J. C. Burgos, "Control of permanent-magnet generators applied to variable-speed wind-energy systems connected to the grid," IEEE Transactions on Energy Conversion, vol. 21, pp. 130-135, March 2006.

[53] S. Zhang, K. J. Tseng, D. M. Vilathgamuwa, T. D. Nguyen, and X. Y. Wang, "Design of a robust grid interface system for pmsg-based wind turbine generators," IEEE Transactions on Industrial Electronics, vol. 58, pp. 316-328, Jan 2011.

[54] J. Grainger and W. Stevenson, Power system analysis. McGraw-Hill series in electrical and computer engineering: Power and energy, McGraw-Hill, 1994.

[55] B. Smith, P. Bjorstad, and W. Gropp, Domain Decomposition: Parallel Multilevel Methods for Elliptic Partial Differential Equations. Cambridge University Press, 2004.

[56] A. Toselli and O. Widlund, Domain Decomposition Methods - Algorithms and Theory. Springer Series in Computational Mathematics, Springer Berlin Heidelberg, 2004.

[57] T. Mathew, Domain Decomposition Methods for the Numerical Solution of Partial Differential Equations. Lecture Notes in Computational Science and Engineering, Springer Berlin Heidelberg, 2008.

[58] H. A. Schwarz, Gesammelte Mathematische Abhandlungen. Springer-Verlag Berlin Heidelberg, 1890.

[59] Y. Fragakis and M. Papadrakakis, "The mosaic of high performance domain decomposition methods for structural mechanics: Formulation, interrelation and numerical efficiency of primal and dual methods," Computer Methods in Applied Mechanics and Engineering, vol. 192, no. 35, pp. 3799 - 3830, 2003. 
[60] A. Klawonn and O. Rheinbach, "Highly scalable parallel domain decomposition methods with an application to biomechanics," ZAMM - Journal of Applied Mathematics and Mechanics / Zeitschrift fr Angewandte Mathematik und Mechanik, vol. 90, no. 1, pp. 5-32, 2010.

[61] A. Jerome, P. Gunupudi, and M. Nakhla, "Efficient parameterized nonlinear simulation of vlsi circuits using domain decomposition techniques," in 2007 IEEE Electrical Performance of Electronic Packaging, pp. 343-346, Oct 2007.

[62] Y. Saad, Iterative Methods for Sparse Linear Systems. Computer Science Series, PWS Publishing Company, 1996.

[63] J. J. Cao, S. S. Fan, and X. Yang, "Spmd performance analysis with parallel computing of matlab," in 2012 Fifth International Conference on Intelligent Networks and Intelligent Systems, pp. 80-83, Nov 2012.

[64] K. Ma, INTRODUCTION TO MATLAB PARALLEL COMPUTING TOOLBOX, 2015.

[65] S. Blumsack, P. Hines, M. Patel, C. Barrows, and E. C. Sanchez, "Defining power network zones from measures of electrical distance," in 2009 IEEE Power Energy Society General Meeting, pp. 1-8, July 2009.

[66] J. M. C'edric Josz, St'ephane Fliscounakis and P. Panciatici, "Ac power flow data in matpower and qcqp format: itesla, rte snapshots, and pegase," Optimization and Control, March 2016.

[67] D. Paul, M. S. Nakhla, R. Achar, and N. M. Nakhla, "Parallel circuit simulation via binary link formulations (pvb)," IEEE Transactions on Components, Packaging and Manufacturing Technology, vol. 3, pp. 768-782, May 2013. 


\section{Appendix A}

\section{Derivation of Power Flow}

\section{A.1 Power Flow Calculation}

Power flow study or load flow study is a steady-state analysis of power system. It is used to determined the voltages, current and power for each bus, each machine and each load. The purpose of power flow studies is to give a brief picture of the whole power system. For example, if one generator is broken, can the remaining system keeps stable without exceeding their rated value. In general, the system is assumed to be balanced, therefore we can use single-phase representation of the system. In this section we will describe the power-flow analysis method, the basic network equations presented here is based on phasor model, which is a little bit different from model in the Chapter. 2.

\section{A.1.1 Bus classification}

A bus in power system is defined as the vertical line where several components are connected. It is similar to Node in circuits. Each bus can be described by four quantities $(P, V, Q, \theta)$, once we know two of them, we can decided the type of buses. The following types are specified: 
- Voltage-controlled (PV) bus: Active power and voltage magnitude are provided. The specific devices are synchronous generators.

- Load (PQ) bus: Active power and reactive power are provided. In general, loads on these bus are assumed to consume constant power, the change of bus voltage are neglected.

- Reference (slack) bus: Voltage magnitude and phase angle are provided. The slack bus is the only bus with known voltage.

\section{A.1.2 Newton-Raphson solution method}

The known and unknown variables in the system are identified by different type of bus. In a system, there are $N$ buses and $R$ generators, the total unknowns are $2(N-1)-(R-1)$. For each bus, we can have following equations, whose number is $2(N-1)-(R-1):$

$$
\begin{aligned}
& P_{i}=V_{i} \sum_{k=1}^{n} V_{k}\left[G_{i k} \cos \left(\theta_{i k}\right)+B_{i k} \sin \left(\theta_{i k}\right)\right] \\
& Q_{i}=V_{i} \sum_{k=1}^{n} V_{k}\left[G_{i k} \sin \left(\theta_{i k}\right)-B_{i k} \cos \left(\theta_{i k}\right)\right]
\end{aligned}
$$

Equations describe the real and reactive power balance for each load bus and generator bus. Using Newton method to solve the Equation. A.1 and A.2.

$$
\left[\begin{array}{c}
\Delta \theta \\
\Delta|V|
\end{array}\right]=-J^{-1}\left[\begin{array}{c}
\Delta P \\
\Delta Q
\end{array}\right]
$$


where

$$
J=\left[\begin{array}{ll}
\frac{\partial \Delta P}{\partial \theta} & \frac{\partial \Delta P}{\partial|V|} \\
\frac{\partial \Delta Q}{\partial \theta} & \frac{\partial \Delta Q}{\partial|V|}
\end{array}\right]
$$

The linearized system of equations A.3 is to determine the next guess of angle and voltage.

$$
\begin{gathered}
\theta^{m+1}=\theta^{m}+\Delta \theta \\
|V|^{m+1}=|V|^{m}+\Delta|V|
\end{gathered}
$$

The iteration stops when the tolerance condition is met. 


\section{Appendix B}

\section{Parameters of the Study Cases}

\section{B.1 IEEE 9 bus system}

Figure. B.1 shows one line diagram of IEEE 9 bus system.

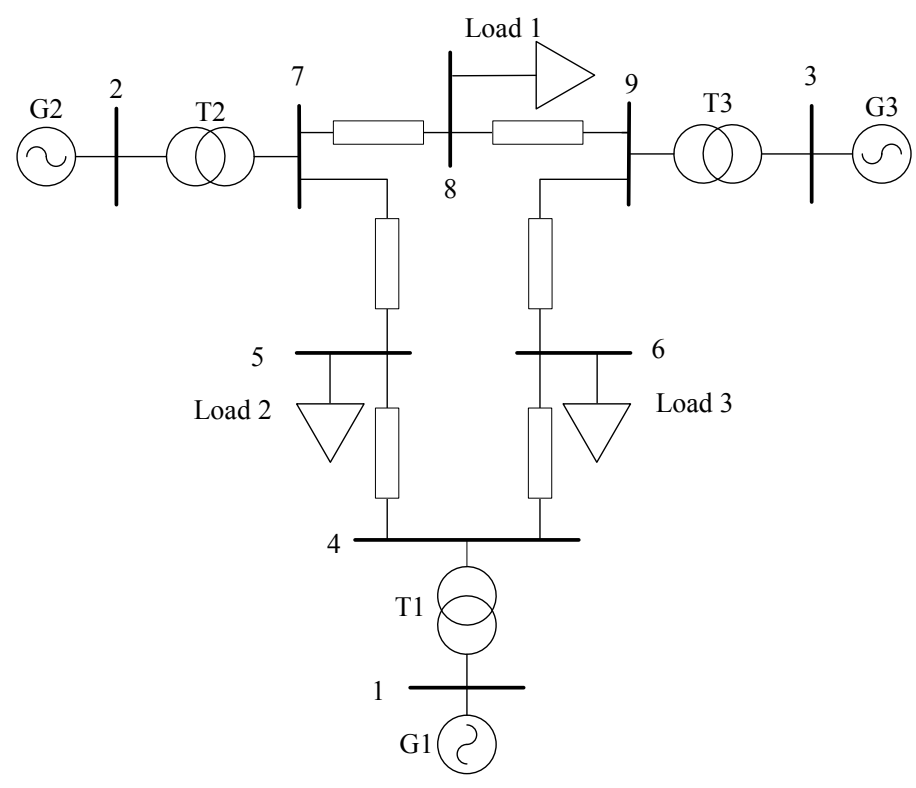

Figure B.1: IEEE standard nine bus system

This system contains nine buses, six transmission lines, three transformers, three 
constant impedance loads, and three synchronous generation machines with IEEE type- 1 exciters. The parameters of the transmission line are given in Table B.1. The parameters of the synchronous machine are given in Table B.2.

Table B.1: Transmission line characteristics of IEEE 9 bus system

\begin{tabular}{|c||c||c|c|c|}
\hline From bus & To bus & $\mathrm{R}(\mathrm{pu} / \mathrm{m})$ & $\mathrm{X}(\mathrm{pu} / \mathrm{m})$ & $\mathrm{B}(\mathrm{pu} / \mathrm{m})$ \\
\hline 4 & 5 & 0.01 & 0.068 & 0.176 \\
\hline 4 & 6 & 0.017 & 0.092 & 0.158 \\
\hline 5 & 7 & 0.032 & 0.161 & 0.306 \\
\hline 6 & 9 & 0.039 & 0.1738 & 0.358 \\
\hline 7 & 8 & 0.0085 & 0.0576 & 0.149 \\
\hline 8 & 9 & 0.0119 & 0.1008 & 0.209 \\
\hline
\end{tabular}

Table B.2: Parameter for each synchronous machines in IEEE 9 bus system

\begin{tabular}{|c||c|c|c|}
\hline $\mathrm{H}$ & 2.6312 & 4.1296 & 4.768 \\
\hline $\mathrm{d}$ & 2 & 2 & 2 \\
\hline$R_{a}$ & 0.004 & 0.0016 & 0.004 \\
\hline$X_{d}$ & 1.7 & 1.7 & 1.22 \\
\hline$X_{d}^{\prime}$ & 0.27 & 0.256 & 0.174 \\
\hline$X_{q}$ & 1.65 & 1.62 & 1.16 \\
\hline$X_{q}^{\prime}$ & 0.47 & 0.245 & 0.25 \\
\hline$T_{d}$ & 3.8 & 4.8 & 8.97 \\
\hline$T_{q}$ & 0.48 & 0.5 & 0.5 \\
\hline$\omega_{\text {ref }}$ & 1 & 1 & 1 \\
\hline$P_{\text {ref }}$ & 0.716 & 1.63 & 0.85 \\
\hline$V_{\text {ref }}$ & 1.04 & 1.025 & 1.025 \\
\hline
\end{tabular}




\section{B.2 IEEE 39 bus system}

The system contains thirty-nine buses, thirty-four transmission lines, eighteen PQ loads and ten generators. Figure. B.2 shows one line diagram of IEEE 39 bus system.

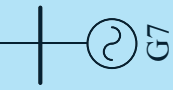

$\dddot{m}$

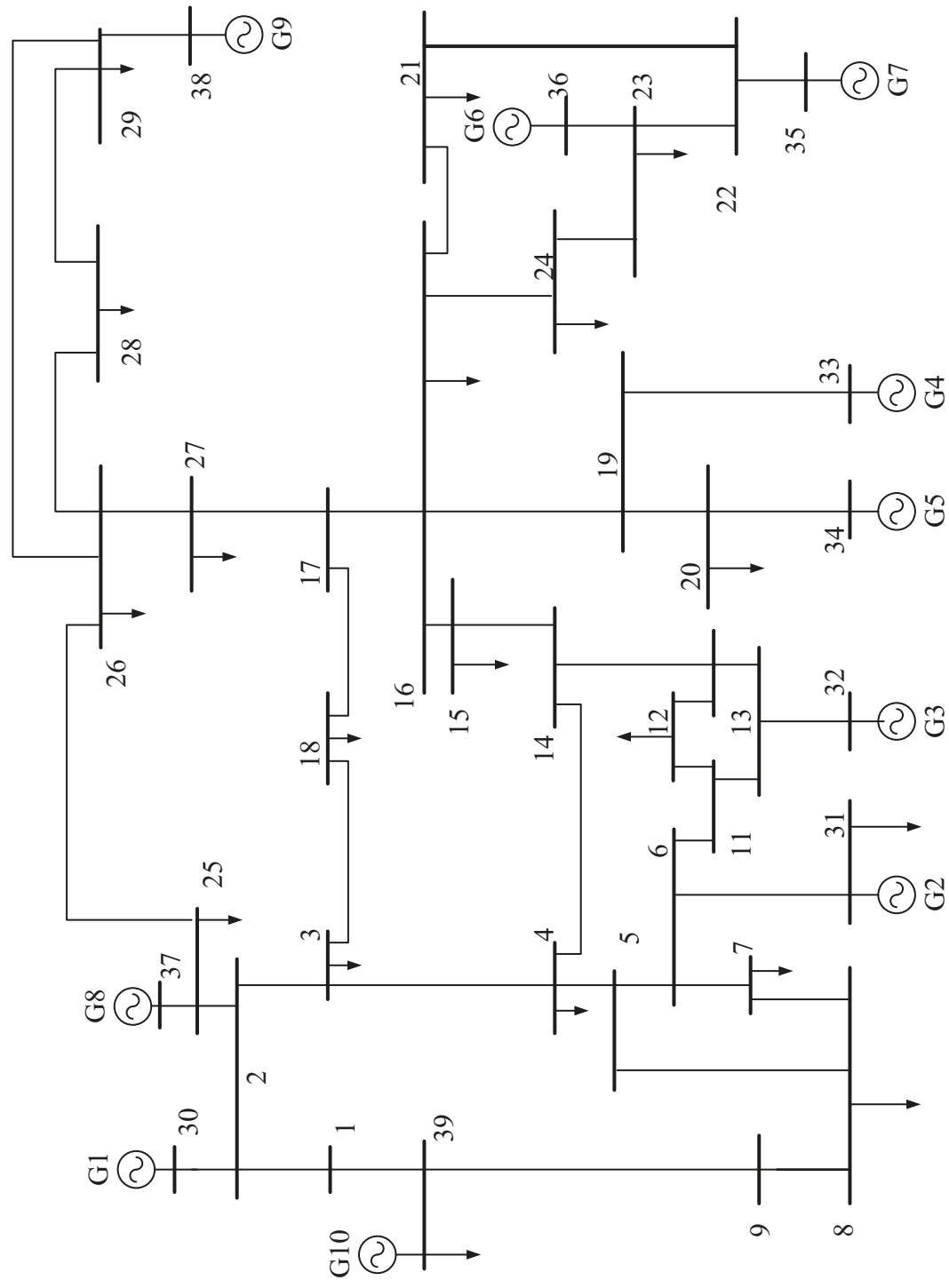

Figure B.2: IEEE standard thirty-nine bus system 
Table B.3: Examples of Transmission line characteristics of IEEE 39 bus system

\begin{tabular}{|c|c|c|c|c|}
\hline From bus & To bus & $\mathrm{R}(\mathrm{pu} / \mathrm{m})$ & $\mathrm{X}(\mathrm{pu} / \mathrm{m})$ & $\mathrm{B}(\mathrm{pu} / \mathrm{m})$ \\
\hline 1 & 2 & 0.0035 & 0.0411 & 0.6987 \\
\hline 1 & 39 & 0.001 & 0.025 & 0.75 \\
\hline 2 & 3 & 0.0013 & 0.0151 & 0.2572 \\
\hline 2 & 25 & 0.007 & 0.0085 & 0.146 \\
\hline 3 & 4 & 0.0013 & 0.0 .0213 & 0.2214 \\
\hline 3 & 18 & 0.0011 & 0.0133 & 0.2138 \\
\hline 4 & 5 & 0.0008 & 0.0128 & 0.1342 \\
\hline 4 & 14 & 0.0008 & 0.0129 & 0.1382 \\
\hline 5 & 6 & 0.0002 & 0.0025 & 0.0434 \\
\hline 5 & 8 & 0.0008 & 0.0112 & 0.1476 \\
\hline 6 & 7 & 0.0006 & 0.0092 & 0.113 \\
\hline 6 & 11 & 0.0007 & 0.0082 & 0.1389 \\
\hline 7 & 8 & 0.0004 & 0.0046 & 0.078 \\
\hline 8 & 9 & 0.0023 & 0.0363 & 0.3804 \\
\hline 9 & 39 & 0.001 & 0.025 & 1.2 \\
\hline 10 & 11 & 0.0004 & 0.0043 & 0.0729 \\
\hline
\end{tabular}




\begin{tabular}{|c|c|c|c|c|}
\hline 10 & 13 & 0.0004 & 0.0043 & 0.0729 \\
\hline 13 & 14 & 0.0009 & 0.0101 & 0.1723 \\
\hline 14 & 15 & 0.0018 & 0.0217 & 0.366 \\
\hline 15 & 16 & 0.0009 & 0.0094 & 0.171 \\
\hline 16 & 17 & 0.0007 & 0.0089 & 0.1342 \\
\hline 16 & 19 & 0.0016 & 0.0195 & 0.304 \\
\hline 16 & 21 & 0.0008 & 0.0135 & 0.2548 \\
\hline 16 & 24 & 0.0003 & 0.0059 & 0.068 \\
\hline 17 & 18 & 0.0007 & 0.0082 & 0.1319 \\
\hline 17 & 27 & 0.0013 & 0.0173 & 0.3216 \\
\hline 21 & 22 & 0.0008 & 0.014 & 0.2565 \\
\hline 22 & 23 & 0.0006 & 0.0096 & 0.1846 \\
\hline 23 & 24 & 0.0022 & 0.035 & 0.361 \\
\hline 25 & 26 & 0.0032 & 0.0323 & 0.513 \\
\hline 26 & 27 & 0.0014 & 0.0147 & 0.2396 \\
\hline 26 & 28 & 0.0043 & 0.0474 & 0.7802 \\
\hline
\end{tabular}




\section{B.3 IEEE 118 bus system}

Figure. B.3 shows one line diagram of IEEE 118 bus system. The system contains one

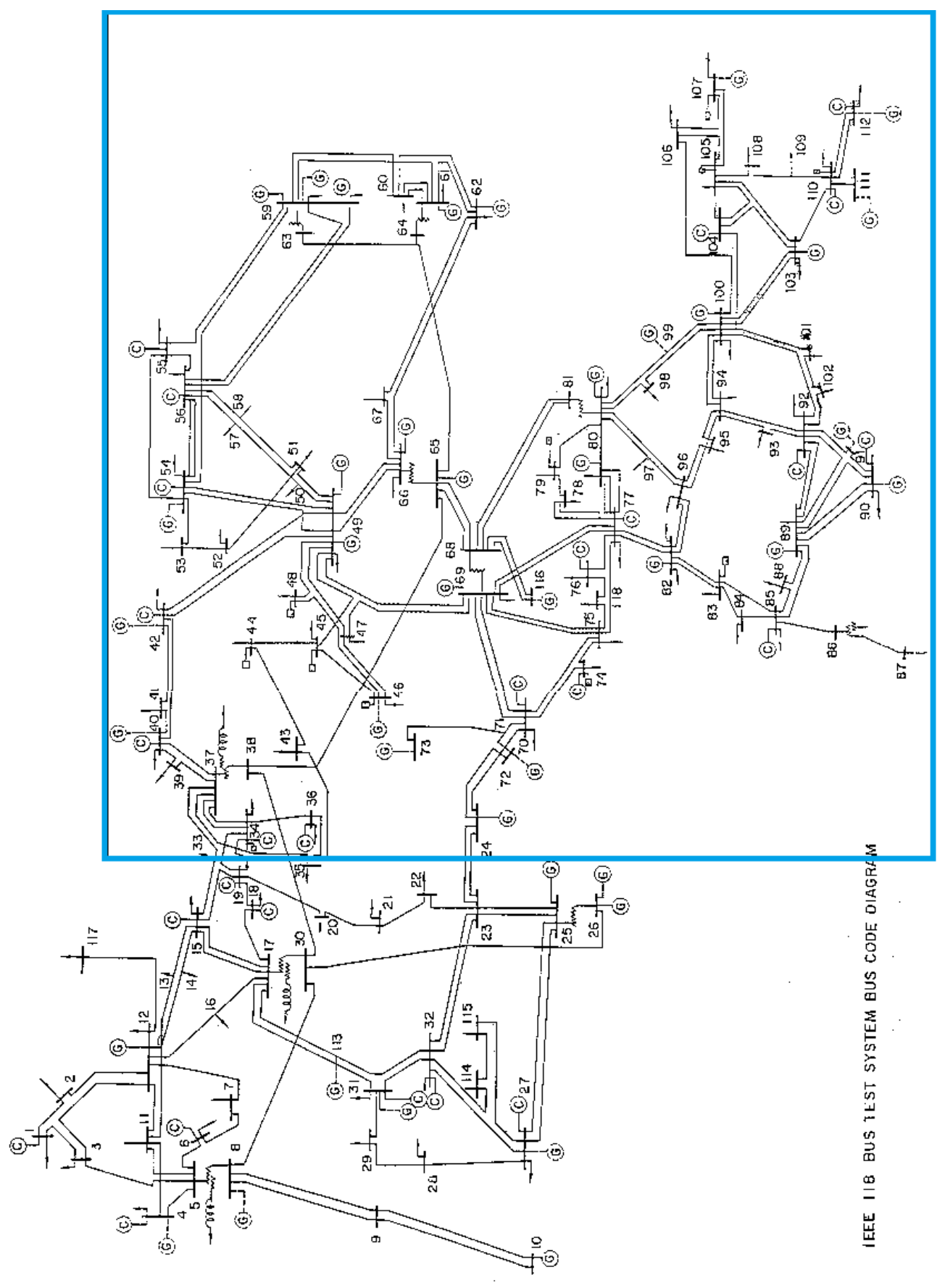

Figure B.3: IEEE standard one hundred eighteen bus system 
hundred and eighteen buses, one hundred and eighty six transmission lines, ninety one PQ loads and fifty four generators. Table. B.4 shows some examples of transmission lines. The entire parameters can be found [66].

Table B.4: Transmission line characteristics of IEEE 118 bus system

\begin{tabular}{|c||c||c|c|c|}
\hline From bus & To bus & $\mathrm{R}(\mathrm{pu} / \mathrm{m})$ & $\mathrm{X}(\mathrm{pu} / \mathrm{m})$ & $\mathrm{B}(\mathrm{pu} / \mathrm{m})$ \\
\hline 1 & 2 & 0.0303 & 0.0999 & 0.0254 \\
\hline 1 & 3 & 0.0129 & 0.0424 & 0.0108 \\
\hline 4 & 5 & 0.018 & 0.008 & 0.0021 \\
\hline 3 & 5 & 0.0241 & 0.108 & 0.0284 \\
\hline 5 & 6 & 0.0119 & 0.054 & 0.0143 \\
\hline 6 & 7 & 0.0046 & 0.0208 & 0.0055 \\
\hline
\end{tabular}

\section{B.4 IEEE 2869 bus system}

The system contains 2869 buses, 4582 transmission lines, 1491 PQ loads and 510 generators. Table. B.5 shows some examples of transmission lines. The entire parameters can be found in testcase of MATPOWER [66].

Table B.5: Transmission line characteristics of IEEE 2869 bus system

\begin{tabular}{|c||c||c|c|c|}
\hline From bus & To bus & $\mathrm{R}(\mathrm{pu} / \mathrm{m})$ & $\mathrm{X}(\mathrm{pu} / \mathrm{m})$ & $\mathrm{B}(\mathrm{pu} / \mathrm{m})$ \\
\hline 5147 & 3097 & $6 e^{-4}$ & 0.0062 & 0 \\
\hline 5147 & 8763 & $5 e^{-4}$ & 0.0074 & 0 \\
\hline 427 & 5425 & $7 e^{-5}$ & $5.7 e^{-4}$ & 0 \\
\hline 427 & 4704 & $6 e^{-5}$ & $5.6 e^{-4}$ & 0 \\
\hline 5960 & 7431 & 0.0033 & 0.0166 & 0 \\
\hline 5960 & 3186 & 0.0034 & 0.016 & 0 \\
\hline 21 & 6163 & 0.00013 & 0.00055 & 0 \\
\hline
\end{tabular}

